\title{
One-Step Diastereoselective Pyrrolidine Synthesis Using a Sulfinamide Annulating Reagent
}

\author{
Qing Shi, ${ }^{* \dagger}$ Nathaniel S. Greenwood, ${ }^{\dagger}$ Mariah C. Meehan ${ }^{\dagger}$ Hyunsoo Park, ${ }^{\ddagger}$ Michael Galella ${ }^{\ddagger}$ \\ Bhupinder Sandhu, ${ }^{\ddagger}$ Purnima Khandelwal, ${ }^{\dagger}$ John R. Coombs, ${ }^{\diamond}$ William P. Gallagher, ${ }^{\diamond}$ Carlos A. \\ Guerrero, ${ }^{\diamond}$ John Hynes Jr., ${ }^{\dagger}$ T. G. Murali Dhar, ${ }^{\dagger}$ Francisco Gonzalez Bobes ${ }^{\diamond}$ and David \\ Marcoux*广 $^{* \dagger}$ \\ ${ }^{\dagger}$ Discovery Chemistry, Bristol-Myers Squibb, Route 206 and Province Line Road, Princeton, New Jersey 08543, \\ United States \\ *Materials Science \& Engineering, Bristol-Myers Squibb, 1 Squibb Drive, New Brunswick, NJ 08903 \\ ${ }^{\ominus}$ Chemical \& Synthetic Development, Bristol-Myers Squibb, 1 Squibb Drive, New Brunswick, NJ 08903
}

Supporting Information

Table of Contents

1. Synthesis of annulating reagents. .2

2. Annulation protocol .5

3. $1 \mathrm{H}$ and $13 \mathrm{C}$ NMR Spectra. .27

\section{General Experiments}

Unless otherwise stated all reactions were run under an atmosphere of nitrogen but glasswares were not flamed dried. Reactions were run in $10 \mathrm{~mL}$ or $25 \mathrm{~mL}$ round bottom flask and monitored on different UPLC/MS instrument. Instrument A: Shimadzu UPLC/MS-2020 (Waters Acquity UPLC BEH C18 column (1.7 um 4.6 x $50 \mathrm{~mm}$ ), solvent A: $95.0 \%$ Water/ 5.0\% Acetonitrile/0.05\% TFA, solvent B: $5.0 \%$ Water/ 95.0\% Acetonitrile/0.05\% TFA, gradient from $10 \%$ B to $100 \%$ B over $1.5 \mathrm{~min}$ then $100 \%$ B for $0.5 \mathrm{~min}$, UV detector $220 \mathrm{~nm}$ ). Instrument B: Waters Acquity SDS (Waters Acquity UPLC BEH C18 column (1.7 um 2.1 x $50 \mathrm{~mm}$ ), solvent A: $100 \%$ Water $/ 0.05 \%$ TFA, solvent B: $100 \%$ Acetonitrile $/ 0.05 \%$ TFA, gradient from $2 \%$ B to $98 \%$ B over 1 min then $98 \%$ B for $0.5 \mathrm{~min}$, UV detector $220 \mathrm{~nm}$ ). Flash column chromatography was performed on a Teledyne ISCO instrument using $12 \mathrm{~g}$ or $24 \mathrm{~g}$ redisep Rf silica column. Hexanes, EtOAc, DCM and MeOH utilised for purification was purchased as UPLC grade from Sigma-Aldrich. Reaction solvents (THF, PhMe, MeCN, DMSO, and DMF) were purchased from Sigma-Aldrich in a Sure/Seal bottle and used without further purification. All reagents and organic building blocks were purchased from commercial supplier (Sigma Aldrich, Alfa Aesar, Combi-blocks) and used without further purification. Vinylsulfones were prepared according to literature procedure. ${ }^{9}$

1H, 13C, and 19F NMR spectra were obtained at $25^{\circ} \mathrm{C}$ on Bruker Avance III and Avance III HD instruments at fields of $400 \mathrm{MHz}, 500 \mathrm{MHz}$ and $700 \mathrm{MHz}$, equipped with $5 \mathrm{~mm}$ BBFO Probe, Prodigy BBO Probe and TCI Cryoprobe, respectively. The spectra were internally referenced to the solvent peak. Chemical shifts are reported in parts per million (ppm). Data is reported in the 
following format: chemical shift $(\delta \mathrm{ppm})$, multiplicity $(\mathrm{s}=$ singlet, $\mathrm{d}=$ doublet, $\mathrm{t}=$ triplet, $\mathrm{q}=$ quartet, $\mathrm{m}=$ multiplet), coupling constant $(\mathrm{Hz})$, integration. High resolution mass spectra (HRMS) were obtained on a ThermoFinnigan LTQ Orbitrap XL (ESI) or a ThermoFinnigan Exactive (ESI).

Specific optical rotations the concentration $c$ is in $\mathrm{g} / 100 \mathrm{~mL}$ and the path length $l$ is in decimeters. The units of the specific rotation, $($ deg.mL)/(g.dm), are implicit and are not included with the reported value.

Single crystal X-ray diffraction measurements were performed at $25^{\circ} \mathrm{C}$ using either Bruker X8 Kappa diffractometer with MicroStarH rotating anode and APEX II detector or Bruker X8 Prospector diffractometer with I $\mu$ S microfocusing source and APEX II detector. Data collection and integration were carried out with APEX2 software. 1 The intensity data were corrected for absorption using SADABS.2 The crystal structures were solved and refined using the software package SHELXTL.3 Full-matrix least squares / non-hydrogen atoms were refined with anisotropic displacement parameters. The hydrogen atoms were placed in ideal positions and refined as riding atoms with relative isotropic displacement parameters.

(R)-1-phenylethyl (2-chloroethyl)carbamate (4b).
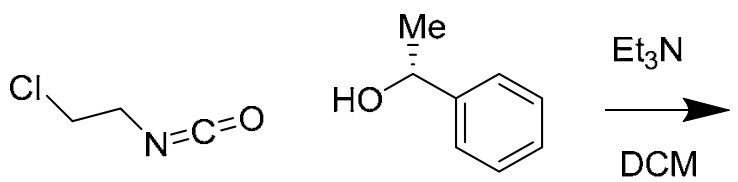<smiles>CC(OC(=O)NCCCl)c1ccccc1</smiles>

A solution of 1-chloro-2-isocyanatoethane (1 g, $9.5 \mathrm{mmol})$, (R)-1-phenylethan-1-ol (1.2 g, 9.5 $\mathrm{mmol})$ and Et3N $(1.6 \mathrm{~mL}, 11 \mathrm{mmol})$ in dichloromethane $(30 \mathrm{~mL})$ was stirred at $\mathrm{rt}$ for 3 days. The reaction mixture was concentrated. The residue was purified using an ISCO teledyne instrument with a $40 \mathrm{~g}$ silica gel column while eluting with hexane and ethyl acetate (gradient of 0 to $100 \%$ ethyl acetate). Fractions containing the desired product were combined and concentrated under reduced pressure to afford the titled compound as a colorless oil ( $1.10 \mathrm{~g}, 51 \%$ yield). UPLC $t r=$ 1.12 min (Instrument A); HRMS (ESI) $\mathrm{m} / z$ calcd for $\mathrm{C} 11 \mathrm{H} 14 \mathrm{ClNO} 2[\mathrm{M}+\mathrm{H}]^{+} 228.0786$, found 228.0787; ${ }^{1} \mathrm{H}$ NMR (CHLOROFORM-d, $\left.400 \mathrm{MHz}\right) \delta 7.27-7.37$ (m, 5H), 5.80 (q, $J=6.4 \mathrm{~Hz}, 1 \mathrm{H}$ ), 5.13 (br s, $1 \mathrm{H}), 3.47-3.62(\mathrm{~m}, 4 \mathrm{H}), 1.55(\mathrm{~d}, J=6.6 \mathrm{~Hz}, 3 \mathrm{H}) ;{ }^{13} \mathrm{C}$ NMR (CHLOROFORM-d, $101 \mathrm{MHz}) \delta 155.8,142.0,128.5(2 \mathrm{C}), 127.9,126.0(2 \mathrm{C}), 73.2,44.2,42.8,22.4 ;[\alpha] \mathrm{D}^{20}=17.7(c=$ 2.50 in DMSO).

(R)-N-(2-chloroethyl)-2-phenylpropanamide (4c).


To a solution of (R)-2-phenylpropanoic acid $(100 \mathrm{mg}, 0.67 \mathrm{mmol})$ in THF $(6.7 \mathrm{~mL})$ at $0{ }^{\circ} \mathrm{C}$ was added 2-chloroethan-1-amine $\mathrm{HCl}$ salt $(150 \mathrm{mg}, 1.3 \mathrm{mmol})$, hunig's base $(230 \mu \mathrm{L}, 1.3 \mathrm{mmol})$ and 
HATU (300 mg, $0.8 \mathrm{mmol}$ ) subsequently. The reaction was stirred at this temperature for $1 \mathrm{~h}$. The reaction mixture was diluted with EtOAc and washed consecutively with $1 \mathrm{~N} \mathrm{HCl}, 1 \mathrm{~N} \mathrm{NaOH}$ and brine affording (R)-N-(2-chloroethyl)-2-phenylpropanamide (120 mg, 85\% yield). UPLC $t r$ $=0.76 \mathrm{~min}$ (Instrument B); HRMS (ESI) $\mathrm{m} / z$ calcd for $\mathrm{C} 11 \mathrm{H} 14 \mathrm{CINO}[\mathrm{M}+\mathrm{H}]^{+} 212.0837$, found 212.0837; ${ }^{1} \mathrm{H}$ NMR (CHLOROFORM-d, 400MHz) $\delta$ 7.27-7.39 (m, 5H), 5.75 (br s, 1H), 3.48$3.63(\mathrm{~m}, 5 \mathrm{H}), 1.54$ (d, $J=7.2 \mathrm{~Hz}, 3 \mathrm{H}) ;{ }^{13} \mathrm{C}$ NMR (CHLOROFORM-d, 101MHz) $\delta 174.5,141.0$, 129.0 (2C), 127.7 (2C), 127.4, 47.1, 43.8, 41.3, 18.5.

(R)-2-chloro-N-(1-phenylethyl)ethan-1-amine (4d).

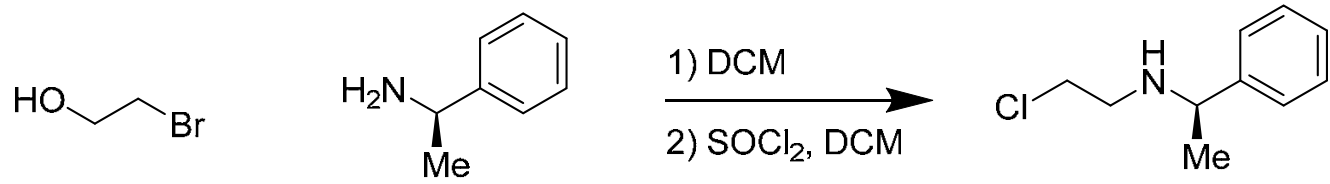

A solution of (R)-1-phenylethan-1-amine (1 g, $8.3 \mathrm{mmol})$ and 2-bromoethan-1-ol $(1.1 \mathrm{~g}, 8.7$ mmol) in DCM (1 mL) was heated at $50{ }^{\circ} \mathrm{C}$ for $\sim 65 \mathrm{~h}$. The reaction mixture was diluted with DCM and washed with saturated solution of $\mathrm{K}_{2} \mathrm{CO}_{3}$. The organic phase was separated and washed with brine and then dried over $\mathrm{Na}_{2} \mathrm{SO}_{4}$. The mixture was filtered and concentrated under reduced pressure. The residue was redissolved in DCM $(10 \mathrm{~mL})$ and warmed to $40{ }^{\circ} \mathrm{C}$. Thionyl chloride $(0.90 \mathrm{~mL}, 12.38 \mathrm{mmol})$ was added dropwise over $10 \mathrm{~min}$ and the resulting solution is heated at $40{ }^{\circ} \mathrm{C}$ overnight. The reaction was cooled to $\mathrm{rt}$ and filtered. The filter cake was washed with DCM ( $5 \mathrm{~mL})$ twice. The solide was dried by sucction affording (R)-2-chloro-N-(1phenylethyl)ethan-1-amine (0.85 g, 57\% yield) as white solid. UPLC $t r=0.54$ min (Instrument B); HRMS (ESI) $m / z$ calcd for $\mathrm{C} 10 \mathrm{H} 14 \mathrm{ClN}[\mathrm{M}+\mathrm{H}]^{+} 184.0888$, found $184.0886 ;{ }^{1} \mathrm{H}$ NMR (400 MHz, METHANOL-d 4 ) $\delta 7.57-7.46(\mathrm{~m}, 5 \mathrm{H}), 4.51$ (q, $J=6.9 \mathrm{~Hz}, 1 \mathrm{H}), 3.89-3.82(\mathrm{~m}, 2 \mathrm{H}), 3.38$ $(\mathrm{dt}, J=13.5,5.8 \mathrm{~Hz}, 1 \mathrm{H}), 3.15(\mathrm{dt}, J=13.6,6.0 \mathrm{~Hz}, 1 \mathrm{H}), 1.75(\mathrm{~d}, J=6.8 \mathrm{~Hz}, 3 \mathrm{H}) ;{ }^{13} \mathrm{C} \mathrm{NMR}(101$ $\mathrm{MHz}$, METHANOL-d4) $\delta 135.8,129.5,129.2(2 \mathrm{C}), 127.5(2 \mathrm{C}), 58.7,47.1,38.6,18.2 ;[\alpha]_{\mathrm{D}}{ }^{20}=$ $3.1(c=8.10$ in $\mathrm{MeOH})$.

General Procedure A: Synthesis of Annulating Reagents. (R)-N-(2-chloroethyl)-2methylpropane-2-sulfinamide (4e)

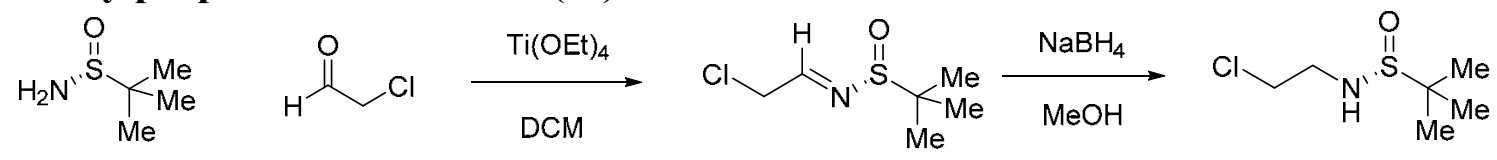

$20 \mathrm{~mL}$ of 2-chloroacetaldehyde ( $\sim 50 \%$ in water) was extracted with dichloromethane (10 mL).

The organic extract was separated, washed with brine, dried with anhyd. $\mathrm{Na}_{2} \mathrm{SO}_{4}$, then Filtered. To the filtrate was added (R)-2-methylpropane-2-sulfinamide (1 g, $8.3 \mathrm{mmol}$ ) followed by tetraethoxytitanium $(3.8 \mathrm{~g}, 17 \mathrm{mmol})$. The reaction mixture was stirred at $\mathrm{rt}$ for $28 \mathrm{~h}$. The reaction mixture was then poured into a vigorously stirring mixture of 1:1 ethyl acetate/brine and stirred for $5 \mathrm{~min}$. The resulting suspension was filtered through Celite and the filter cake was washed with ethyl acetate for 3 times. The organic phase of the filtrate was separated and concentrated. The residue was purified using an ISCO teledyne instrument with a $80 \mathrm{~g}$ silica gel column while eluting with hexanes and EtOAc (gradient of 0 to 100\% EtOAc). Fractions containing the desired product were combined and concentrated under reduced pressure to give 
(R,E)-N-(2-chloroethylidene)-2-methylpropane-2-sulfinamide (0.86 g, 57\% yield) as colorless oil. 1H NMR (499MHz, CHLOROFORM-d) $\delta$ 8.02-8.06 (m, 1H), 4.30-4.33 (m, 2H), 1.22 ppm $(\mathrm{s}, 9 \mathrm{H})$. To a solution of (R,E)-N-(2-chloroethylidene)-2-methylpropane-2-sulfinamide $(1.1 \mathrm{~g}$, $6.05 \mathrm{mmol})$ in $\mathrm{MeOH}(20 \mathrm{~mL})$ cooled in ice-water bath was added NaBH4 $(0.458 \mathrm{~g}, 12.11$ $\mathrm{mmol})$ portionwise over $10 \mathrm{~min}$. Stirring was continued at this temperature when the reaction was deemed completed by LC/MS analysis. The reaction was quenched with satd. $\mathrm{NH} 4 \mathrm{Cl}$ (3 $\mathrm{mL})$, extracted between water $(20 \mathrm{~mL})$ and ethyl acetate $(20 \mathrm{~mL})$. The aqueous phase was extracted with more ethyl acetate $(20 \mathrm{~mL})$. Combined organic phases was washed with water, brine, dried over anhyd. Na2SO4. Filtered and concentrated to give (R)-N-(2-chloroethyl)-2methylpropane-2-sulfinamide $(0.98 \mathrm{~g}, 88 \%$ yield $)$ as white solid. UPLC $t r=0.66 \mathrm{~min}$ (Instrument B); HRMS (ESI) $\mathrm{m} / z$ calcd for C6H15CINOS $[\mathrm{M}+\mathrm{H}]^{+} 184.0557$, found 184.0559; ${ }^{1} \mathrm{H}$ NMR (400 MHz, CHLOROFORM-d) $\delta 3.77$ - $3.63(\mathrm{~m}, 3 \mathrm{H}), 3.62$ - $3.41(\mathrm{~m}, 2 \mathrm{H}), 1.31$ - 1.16 $(\mathrm{m}, 9 \mathrm{H}) ;{ }^{13} \mathrm{C}$ NMR $\left(101 \mathrm{MHz}\right.$, CHLOROFORM-d) $\delta 56.1,47.4,45.1,22.6(3 \mathrm{C}) ;[\alpha]_{\mathrm{D}}{ }^{20}=-32(c=$ 5.50 in $\left.\mathrm{CHCl}_{3}\right)$.

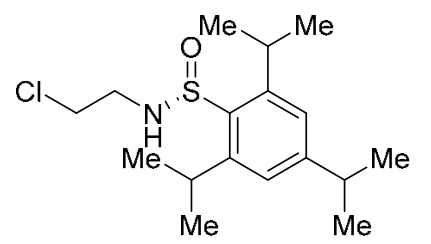

(R)-N-(2-chloroethyl)-2,4,6-triisopropylbenzenesulfinamide (4f). Compound was synthesized according to General Procedure A. The product was purified using an ISCO teledyne instrument with a $40 \mathrm{~g}$ silica gel column while eluting with dichloromethane and $\mathrm{MeOH}$ (gradient of 0 to $10 \% \mathrm{MeOH})$ and was isolated a white solid $(1.07 \mathrm{~g}$, $87 \%$ yield). UPLC $t r=1.54 \mathrm{~min}$ (Instrument A); HRMS (ESI) $\mathrm{m} / z$ calcd for C17H28CINOS [M+H] ${ }^{+} 330.1653$, found 330.1656; ${ }^{1} \mathrm{H}$ NMR (CHLOROFORM-d, $400 \mathrm{MHz}) \delta$ 7.06-7.11 (m, 2H), 4.78-4.85 (m, 1H), 3.97-4.09 (m, 2H), 3.79-3.86 (m, 1H), 3.62$3.76(\mathrm{~m}, 2 \mathrm{H}), 3.48-3.59(\mathrm{~m}, 1 \mathrm{H}), 2.88(\mathrm{dt}, J=13.8,6.9 \mathrm{~Hz}, 1 \mathrm{H}), 1.33(\mathrm{~d}, J=6.8 \mathrm{~Hz}, 6 \mathrm{H}), 1.25(\mathrm{t}$, $J=7.0 \mathrm{~Hz}, 12 \mathrm{H}) ;{ }^{13} \mathrm{C}$ NMR (CHLOROFORM-d, 101MHz) $\delta 152.1,147.6(2 \mathrm{C}), 137.9,123.1$ $(2 \mathrm{C}), 47.8,45.6,34.4,28.4(2 \mathrm{C}), 24.4(2 \mathrm{C}), 24.2(2 \mathrm{C}), 23.8(2 \mathrm{C}) ;[\alpha]_{\mathrm{D}}^{20}=-37(c=2.50$ in DMSO).

\section{(R)-N-(3-chloropropyl)-2-methylpropane-2-sulfinamide (4g).}



To a solution of 3-chloropropan-1-amine hydrochloride (5 g, $38.5 \mathrm{mmol})$ in DCM (100 mL) cooled in ice-water bath was added dropwise addtion of Et3N (13.40 mL, $96 \mathrm{mmol})$ and 2methylpropane-2-sulfinic chloride $(5.41 \mathrm{~g}, 38.5 \mathrm{mmol})$ at the same time. Stirred at 0 degree to $\mathrm{rt}$ for $16 \mathrm{~h}$. The reaction mixture was washed with water then brine then concentrated. The product was purified using an ISCO teledyne instrument with a $80 \mathrm{~g}$ silica gel column while eluting with hexane and ethyl acetate (gradient of 0 to $100 \%$ ethyl acetate) and N-(3-chloropropyl)-2methylpropane-2-sulfinamide was isolated as yellow thick oil (4.70 g, 62\% yield). The racemic mixture was then separated using SFC (Column: AD-H $\left(5^{\prime} 25 \mathrm{~cm}, 5 \mu \mathrm{m}\right.$, \#AD321), BPR pressure: 100 bars, Temperature: $35^{\circ} \mathrm{C}$, Flow rate: $300 \mathrm{~mL} / \mathrm{min}$, Mobile Phase: $\mathrm{CO} 2 / \mathrm{MeOH}$ w 0.1\% $\mathrm{NH} 4 \mathrm{OH}$ (80/20), Detector Wavelength: $220 \mathrm{~nm}$, Separation Program: stack injection, Injection: $0.5 \mathrm{~mL}$ with cycle time $1.5 \mathrm{mins}$, Sample preparation : $6.7 \mathrm{~g} / 40 \mathrm{~mL} \mathrm{MeOH}, 167.5 \mathrm{mg} / \mathrm{mL}$, Throughput: $3.3 \mathrm{~g} / \mathrm{hr}$ ). The slow eluting peak was tentatively assigned as $\mathrm{R}$ isomer based on 
previous experiments with similiar sulfinamides that slow eluting isomer has the $\mathrm{R}$ configuration. The characterization of the slow eluting isomer: UPLC $t r=0.85 \mathrm{~min}$ (by mass detector on Instrument A); HRMS (ESI) $m / z$ calcd for C7H16CINOS $[\mathrm{M}+\mathrm{H}]^{+} 198.0714$, found 198.0716; ${ }^{1} \mathrm{H}$ NMR (CHLOROFORM-d, 499MHz) $\delta 3.62-3.67$ (m, 2H), 3.27-3.41 (m, 3H), 2.00-2.09 (m, 2H), 1.21-1.24 ppm (m, 9H) ${ }^{13} \mathrm{C}$ NMR (CHLOROFORM-d, 101MHz) $\delta 55.8$, $42.7,42.0,33.4,22.6(3 \mathrm{C}) ;[\alpha] \mathrm{D}^{20}=48(c=4.36$ in $\mathrm{AcCN})$.

\section{(R)-N-(3-bromopropyl)-2-methylpropane-2-sulfinamide (4h).}

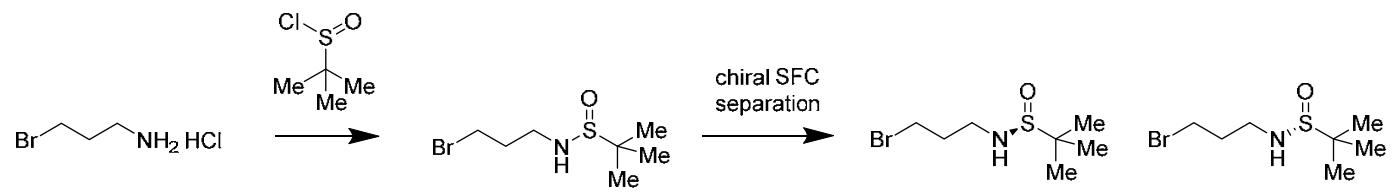

To a solution of 3-bromopropan-1-amine hydrochloride (5 g, $28.7 \mathrm{mmol})$ in DCM ( $5 \mathrm{~mL})$ cooled in ice-water bath was added Et3N (9.99 mL, $71.6 \mathrm{mmol})$. After stirring for $5 \mathrm{~min}$. 2methylpropane-2-sulfinic chloride $(4.03 \mathrm{~g}, 28.7 \mathrm{mmol})$ was added dropwise. Stirred at 0 degree to $\mathrm{rt}$ for $16 \mathrm{~h}$. The reaction mixture was washed with water then brine then concentrated. The residue was purified by $\mathrm{mPLC}$ (ISCO, $0-100 \% \mathrm{EA} /$ hexane, $80 \mathrm{~g}$ silica gel column). Got $4.5 \mathrm{~g}$ yellow oil. The product was purified using an ISCO teledyne instrument with a $80 \mathrm{~g}$ silica gel column while eluting with hexane and ethyl acetate (gradient of 0 to $100 \%$ ethyl acetate) and N(3-bromopropyl)-2-methylpropane-2-sulfinamide was isolated as yellow thick oil (4.50 g, 65\% yield). The racemic mixture was then separated using SFC (Column: AD-H $\left(5^{\prime} 25 \mathrm{~cm}, 5 \mu \mathrm{m}\right.$, \#AD321), BPR pressure: 100 bars, Temperature: $35^{\circ} \mathrm{C}$, Flow rate: $350 \mathrm{~mL} / \mathrm{min}$, Mobile Phase: $\mathrm{CO} 2 / \mathrm{MeOH}$ w 0.1\% NH4OH (75/25), Detector Wavelength: $220 \mathrm{~nm}$, Separation Program: stack injection, Injection: $0.75 \mathrm{~mL}$ with cycle time $1.45 \mathrm{mins}$, Sample preparation: $4.5 \mathrm{~g} / 35 \mathrm{~mL} \mathrm{MeOH}$, $128.57 \mathrm{mg} / \mathrm{mL}$, Throughput: $3.9 \mathrm{~g} / \mathrm{hr}$ ). The slow eluting peak was tentatively assigned as $\mathrm{R}$ isomer based on previous experience with similiar sulfinamides that slow eluting isomer has the $\mathrm{R}$ configuration. The characterization of the slow eluting isomer: UPLC $t r=0.89 \mathrm{~min}$ (by mass detector on Instrument A); HRMS (ESI) $m / z$ calcd for C7H16BrNOS $[\mathrm{M}+\mathrm{H}]^{+} 242.0209$, found 242.0213; ${ }^{1} \mathrm{H}$ NMR (CHLOROFORM-d, 400MHz) $\delta 3.49$ (t, $\left.J=6.4 \mathrm{~Hz}, 2 \mathrm{H}\right), 3.18-3.43$ (m, 3H), 2.06-2.18 (m, 2H), $1.22 \mathrm{ppm}(\mathrm{s}, 9 \mathrm{H}) ;{ }^{13} \mathrm{C}$ NMR (CHLOROFORM-d, 101MHz) $\delta 55.8,43.8$, $33.4,30.4,22.6(3 \mathrm{C}) ;[\alpha]_{\mathrm{D}}^{20}=10.5(c=2.87$ in $\mathrm{AcCN})$.

\section{General Procedure B: Annulation reaction.}

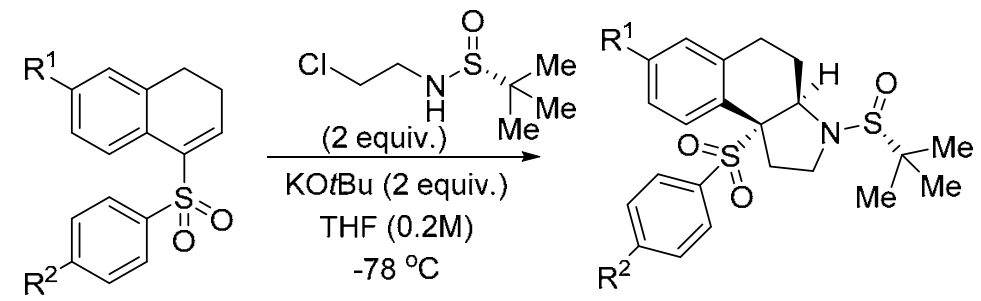

To a stirred solution of electrophile $(0.4 \mathrm{mmol})$ and $(\mathrm{R})-\mathrm{N}-(2-c h l o r o e t h y l)-2-m e t h y l p r o p a n e-2-$ sulfinamide $(0.8 \mathrm{mmol})$ in a $10 \mathrm{~mL}$ round bottom flask was added THF $(1.2 \mathrm{~mL})$. The resulting solution was cooled using a dry ice-acetone bath and stirred at this temperature for $15 \mathrm{~min}$. To 
this reaction mixture was added a $1 \mathrm{~N}$ THF solution of $\mathrm{KO} t \mathrm{Bu}(0.8 \mathrm{mmol})$ dropwise and the reaction was stirred in the dry ice-acetone bath. The reaction progress was monitored by UPLC/MS. In general, the reaction was completed in $30 \mathrm{~min}$. as reveiled by the disappearance of vinyl sulfone starting material but was allowed to stir for an additional $30 \mathrm{~min}$. in the dry iceacetone bath. After completion, the reaction was quenched with $2 \mathrm{~mL}$ of $1 \mathrm{~N} \mathrm{HCl}$ and diluted with EtOAc. The organic layer was separated and the aqueous layer was extracted once with EtOAc. The organic layers were combined and washed with water once then brine once. The organic layer was separated and concentrated. Crude $1 \mathrm{H}$ NMR was obtained. All material was purified using an ISCO teledyne instrument with a $12 \mathrm{~g}$ silica gel column while eluting with hexanes and EtOAc (gradient of 0 to $100 \%$ EtOAc). Fractions containing the desired product were combined and concentrated under reduced pressure.

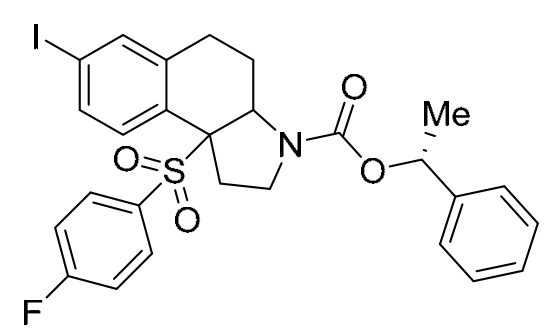

(R)-1-phenylethyl 9b-((4-fluorophenyl)sulfonyl)-7-iodo1,2,3a,4,5,9b-hexahydro-3H-benzo[e]indole-3-carboxylate (3b). Compound was synthesized according to General Procedure B except that the reaction temperature was kept at 0 ${ }^{\circ} \mathrm{C}$. The product was purified using an ISCO teledyne instrument with a $12 \mathrm{~g}$ silica gel column while eluting with hexanes and EtOAc (gradient of 0 to $100 \%$ EtOAc) and was isolated as a white solid (175 mg, 71\% yield). Diastereoisomers were separated using SFC (Column: Chiral ID-H 250 X 4.6 mm ID, $5 \mu \mathrm{m}$, Flow rate: $3.0 \mathrm{~mL} / \mathrm{min}$, Mobile Phase: 90/10 CO2/MeOH).

Data for isomer 1 (first eluting peak on SFC separation): UPLC $t r=1.63 \mathrm{~min}$ (Instrument A); HRMS (ESI) $m / z$ calcd for C27H25FINO4S [M+NH4] ${ }^{+} 623.0871$, found $623.0888 ;{ }^{1} \mathrm{H}$ NMR (DMSO-d $\left., 100{ }^{\circ} \mathrm{C}, 400 \mathrm{MHz}\right) \delta$ 7.60-7.69 (m, 1H), 7.44-7.55 (m, 3H), 7.25-7.43 (m, 8H), 5.77 (q, $J=6.4 \mathrm{~Hz}, 1 \mathrm{H}), 4.45-4.60(\mathrm{~m}, 1 \mathrm{H}), 3.51-3.63(\mathrm{~m}, 1 \mathrm{H}), 3.39-3.51(\mathrm{~m}, 1 \mathrm{H}), 3.25$ (ddd, $J=14.6$, 8.2, 3.6 Hz, 1H), 2.86-2.99 (m, 1H), 2.40-2.59 (m, 3H), 2.22-2.34 (m, 1H), 1.94-2.07 (m, 1H), 1.49-1.61 (m, 3H), 1.28-1.44 ppm (m, 1H); ${ }^{13} \mathrm{C}$ NMR (DMSO-d $\left., 100{ }^{\circ} \mathrm{C}, 101 \mathrm{MHz}\right): \delta 166.0(\mathrm{~d}$, $J=257 \mathrm{~Hz}, 1 \mathrm{C}), 153.9,142.7,142.6,137.0,135.8,133.2$ (d, $J=11 \mathrm{~Hz}, 2 \mathrm{C}), 132.6,132.5$ (d, $J=4.0$ $\mathrm{Hz}, 1 \mathrm{C}), 128.8$ (2C), 127.9, 126.1 (2C), 116.7 (d, $J=23 \mathrm{~Hz}, 2 \mathrm{C}), 95.2,76.2,73.1,60.1$, 45.2, $33.9,27.6,27.2,22.9 \mathrm{ppm} ;[\alpha]_{\mathrm{D}}^{20}=-81\left(c=2.50\right.$ in $\left.\mathrm{CHCl}_{3}\right)$.

Data for isomer 2 (second eluting peak on SFC separation): UPLC $t r=1.63 \mathrm{~min}$ (Instrument A); HRMS (ESI) $m / z$ calcd for C27H25FINO4S [M+NH4] ${ }^{+} 623.0871$, found $623.0882 ;{ }^{1} \mathrm{H} \mathrm{NMR}$ $\left(\mathrm{DMSO}_{6}, 100{ }^{\circ} \mathrm{C}, 400 \mathrm{MHz}\right) \delta$ 7.58-7.69 (m, 1H), 7.41-7.54 (m, 3H), 7.22-7.40 (m, 8H), 5.69$5.80(\mathrm{~m}, 1 \mathrm{H}), 4.45-4.57(\mathrm{~m}, 1 \mathrm{H}), 3.37-3.61(\mathrm{~m}, 2 \mathrm{H}), 3.15-3.30(\mathrm{~m}, 1 \mathrm{H}), 2.86-2.98(\mathrm{~m}, 1 \mathrm{H}), 2.38-$ $2.58(\mathrm{~m}, 3 \mathrm{H}), 2.18-2.32(\mathrm{~m}, 1 \mathrm{H}), 1.89-2.06(\mathrm{~m}, 1 \mathrm{H}), 1.46-1.58(\mathrm{~m}, 3 \mathrm{H}), 1.25-1.42 \mathrm{ppm}(\mathrm{m}, 1 \mathrm{H})$; ${ }^{13} \mathrm{C}$ NMR (DMSO-d $\left.6,100{ }^{\circ} \mathrm{C}, 101 \mathrm{MHz}\right): \delta 166.0$ (d, $\left.J=257 \mathrm{~Hz}, 1 \mathrm{C}\right), 153.9,142.7,142.6,137.0$, $135.8,133.2$ (d, $J=11 \mathrm{~Hz}, 2 \mathrm{C}), 132.6,132.5$ (d, $J=6 \mathrm{~Hz}, 1 \mathrm{C}), 128.8$ (2C), 127.9, 126.1 (2C), $116.7(\mathrm{~d}, J=23 \mathrm{~Hz}, 2 \mathrm{C}), 95.2,76.2,73.1,60.0,45.2,33.9,27.6,27.2,22.9 \mathrm{ppm} ;[\alpha]_{\mathrm{D}}{ }^{20}=85.6(c=$ 2.50 in $\left.\mathrm{CHCl}_{3}\right)$. 
<smiles>CC(C)(C)[S@](=O)N1CC[C@]2(S(=O)(=O)c3ccc(F)cc3)c3ccc(I)cc3CC[C@H]12</smiles>

(3aR,9bR)-3-((R)-tert-butylsulfinyl)-9b-((4fluorophenyl)sulfonyl)-7-iodo-2,3,3a,4,5,9b-hexahydro- $1 \mathrm{H}-$ benzo[e]indole (3e). Compound was synthesized according to General Procedure B. The product was purified using an ISCO teledyne instrument with a $12 \mathrm{~g}$ silica gel column while eluting with hexanes and EtOAc (gradient of 0 to $100 \%$ EtOAc) and was isolated as a white solid ( $175 \mathrm{mg}, 78 \%$ yield). UPLC $t r=1.51 \mathrm{~min}$

(Instrument A); HRMS (ESI) $\mathrm{m} / z$ calcd for $\mathrm{C} 22 \mathrm{H} 25 \mathrm{FINO} 3 \mathrm{~S} 2[\mathrm{M}+\mathrm{H}]^{+} 562.0377$, found 562.0378; ${ }^{1} \mathrm{H}$ NMR (CHLOROFORM-d, 400MHz): $\delta=7.57$ (dd, $\left.J=8.5,1.9 \mathrm{~Hz}, 1 \mathrm{H}\right), 7.44-7.51$ (m, 2H), $7.43(\mathrm{~d}, J=1.7 \mathrm{~Hz}, 1 \mathrm{H}), 7.22-7.29(\mathrm{~m}, 1 \mathrm{H}), 7.07-7.14(\mathrm{~m}, 2 \mathrm{H}), 4.41$ (dd, $J=7.2,4.4 \mathrm{~Hz}$, $1 \mathrm{H}), 3.88$ (ddd, $J=10.6,8.1,3.6 \mathrm{~Hz}, 1 \mathrm{H}), 2.94(\mathrm{dt}, J=12.8,8.5 \mathrm{~Hz}, 1 \mathrm{H}), 2.58-2.68(\mathrm{~m}, 2 \mathrm{H}), 2.33-$ $2.42(\mathrm{~m}, 1 \mathrm{H}), 2.13-2.23(\mathrm{~m}, 1 \mathrm{H}), 1.89(\mathrm{td}, J=9.0,4.3 \mathrm{~Hz}, 1 \mathrm{H}), 1.57-1.67(\mathrm{~m}, 1 \mathrm{H}), 1.15 \mathrm{ppm}(\mathrm{s}$, 9H); ${ }^{13} \mathrm{C}$ NMR (CHLOROFORM-d, 101MHz): $\delta 166.1$ (d, $\left.J=260 \mathrm{~Hz}, 1 \mathrm{C}\right), 142.3,137.4,135.6$, 133.1 (d, $J=10 \mathrm{~Hz}, 2 \mathrm{C}), 132.0,131.9,130.1,116.2$ (d, $J=20 \mathrm{~Hz}, 2 \mathrm{C}), 95.2,73.6,64.0,57.5,40.1$, 36.6, 26.7, 24.9, $23.5(3 \mathrm{C}) ;[\alpha]_{\mathrm{D}}^{20}=6.7(c=7.75$ in $\mathrm{CHCl} 3)$. The dr was $49: 1$ determined by analytical SFC (Column: Chiralpak ID $\left(0.46^{\prime} 25 \mathrm{~cm}, 5 \mu \mathrm{m}\right)$, BPR pressure: 140 bars, Temperature: $40{ }^{\circ} \mathrm{C}$, Flow rate: $3 \mathrm{~mL} / \mathrm{min}$, Mobile Phase: $\mathrm{CO} 2 / \mathrm{MeOH}$ (60/40), Detector Wavelength: $220 \mathrm{~nm}$ )

\section{X-ray Crystallography of $3 e$}

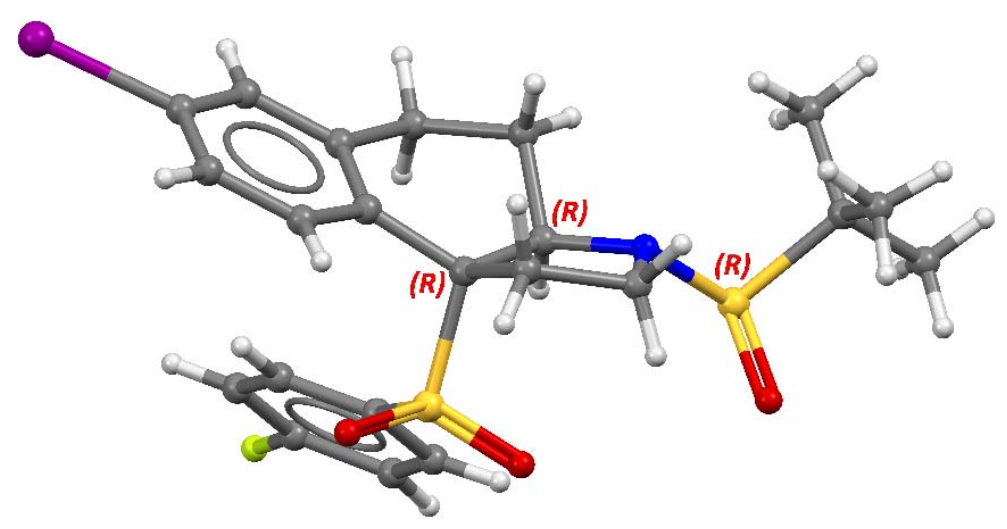

The single crystal X-ray data was collected from large colorless prisms from an ethyl acetate solution. Analysis of the anomalous scattering signal illustrates the above configuration.

\section{Crystal data and structure refinement for $3 e$}








9b-((4-fluorophenyl)sulfonyl)-7-iodo-2,3,3a,4,5,9b-hexahydro-1Hbenzo[e]indole (S1). (3aR,9bR)-3-((R)-tert-butylsulfinyl)-9b-((4fluorophenyl)sulfonyl)-7-iodo-2,3,3a,4,5,9b-hexahydro-1H-benzo[e]indole (3e) $(40 \mathrm{mg}, 0.071 \mathrm{mmol})$ was treated with $4 \mathrm{~N} \mathrm{HCl}$ in dioxane $(1 \mathrm{~mL})$ for $2.5 \mathrm{~h}$ and stirred at room temperature. The reaction mixture was concentrated and utilized without further purification. The dr of 49:1 was determined by analytical SFC of the crude reaction mixture (Column: ChromegaChiral CC4 $\left(0.46^{\prime} 25 \mathrm{~cm}, 5 \mu \mathrm{m}\right)$, BPR pressure: 140 bars,

Temperature: $40{ }^{\circ} \mathrm{C}$, Flow rate: $3 \mathrm{~mL} / \mathrm{min}$, Mobile Phase: $\mathrm{CO} 2 / \mathrm{MeOH}$ with $0.1 \% \mathrm{NH} 4 \mathrm{OH}$ (70/30), Detector Wavelength: $220 \mathrm{~nm}$ ). UPLC $t r=1.11 \mathrm{~min}$ (Instrument A); HRMS (ESI) $\mathrm{m} / z$ calcd for C18H17FINO2S [M+H] ${ }^{+} 458.0078$, found 458.0081; ${ }^{1} \mathrm{H}$ NMR (CHLOROFORM-d, $400 \mathrm{MHz}) \delta 7.59(\mathrm{dd}, J=8.4,1.1 \mathrm{~Hz}, 1 \mathrm{H}), 7.25-7.34(\mathrm{~m}, 4 \mathrm{H}), 6.99-7.06(\mathrm{~m}, 2 \mathrm{H}), 3.95(\mathrm{dd}$, $J=12.0,5.8 \mathrm{~Hz}, 1 \mathrm{H}), 3.31(\mathrm{ddd}, J=11.7,7.7,4.2 \mathrm{~Hz}, 1 \mathrm{H}), 3.18$ (dt, $J=13.7,7.8 \mathrm{~Hz}, 1 \mathrm{H}), 2.99$ (dt, $J=11.6,7.4 \mathrm{~Hz}, 1 \mathrm{H}), 2.30-2.41$ (m, 2H), 2.16 (br s, 1H), 1.97 (ddt, $J=12.9,5.8,3.6 \mathrm{~Hz}, 1 \mathrm{H})$, 1.60-1.74 (m, 1H), 1.24-1.38 ppm (m, 1H); ${ }^{13} \mathrm{C}$ NMR (CHLOROFORM-d, 101MHz) $\delta 165.9(\mathrm{~d}$, $J=260 \mathrm{~Hz}, 1 \mathrm{C}), 143.0,136.7,135.6,133.5,132.9,132.9$ (d, $J=9 \mathrm{~Hz}, 2 \mathrm{C}), 131.6$ (d, $J=4 \mathrm{~Hz}, 1 \mathrm{C})$, 
$115.8(\mathrm{~d}, J=23 \mathrm{~Hz}, 2 \mathrm{C}), 94.5,77.7,62.2,45.9,38.7,28.5,27.7 ;[\alpha]_{\mathrm{D}}^{20}=104.79(c=2.62$ in $\mathrm{AcCN})$.

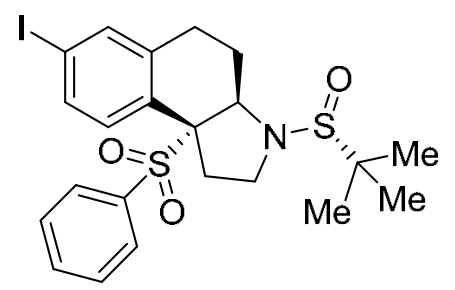

(3aR,9bR)-3-((R)-tert-butylsulfinyl)-9b-((4chlorophenyl)sulfonyl)-7-iodo-2,3,3a,4,5,9b-hexahydro-1Hbenzo[e]indole (7a). Compound was synthesized according to General Procedure B. The product was purified using an ISCO teledyne instrument with a $12 \mathrm{~g}$ silica gel column while eluting with hexanes and EtOAc (gradient of 0 to $100 \%$ EtOAc) and was isolated as a white solid (173 $\mathrm{mg}, 75 \%$ yield). UPLC $t r=1.56 \mathrm{~min}$

(Instrument A); HRMS (ESI) $\mathrm{m} / z$ calcd for C22H25ClINO3S2 [M+H] ${ }^{+} 578.0082$, found 578.0090; ${ }^{1} \mathrm{H}$ NMR (400 MHz, CHLOROFORM-d) $\delta 7.59$ (dd, $J=8.4,1.8 \mathrm{~Hz}, 1 \mathrm{H}$ ), $7.46-7.25$ (m, 6H), 4.44 (dd, $J=7.2,4.4 \mathrm{~Hz}, 1 \mathrm{H}), 3.95$ - 3.87 (m, 1H), 2.96 (dt, $J=12.8,8.6 \mathrm{~Hz}, 1 \mathrm{H}), 2.70$ $2.60(\mathrm{~m}, 2 \mathrm{H}), 2.48-2.33(\mathrm{~m}, 1 \mathrm{H}), 2.27-2.15(\mathrm{~m}, 1 \mathrm{H}), 1.91$ (ddt, $J=13.5,8.9,4.5 \mathrm{~Hz}, 1 \mathrm{H}), 1.78-$ 1.59 (m, 1H), 1.17 (s, 9H); ${ }^{13} \mathrm{C}$ NMR (101 MHz, CHLOROFORM-d) $\delta 142.2,141.1,137.4$, 135.6, 134.4, 131.9 (2C), 131.7, 130.0, 129.1 (2C), 95.3, 73.6, 64.0, 57.6, 40.1, 36.6, 26.6, 24.9, $23.5(3 \mathrm{C}) ;[\alpha]_{\mathrm{D}}^{20}=3.4(c=3.15$ in $\mathrm{CHCl} 3)$.

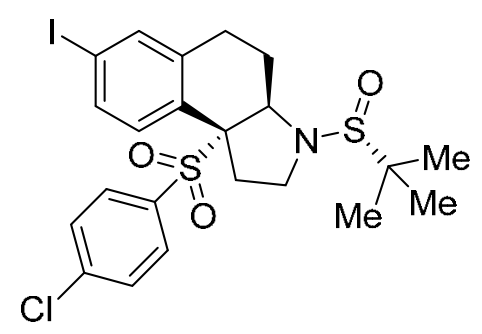

(3aR,9bR)-3-((R)-tert-butylsulfinyl)-7-iodo-9b-((4chlorophenyl)sulfonyl)-2,3,3a,4,5,9b-hexahydro-1Hbenzo[e]indole (7b). Compound was synthesized according to General Procedure B. The product was purified using an ISCO teledyne instrument with a $12 \mathrm{~g}$ silica gel column while eluting with hexanes and EtOAc (gradient of 0 to $100 \%$ EtOAc) and was isolated as a white solid ( $133 \mathrm{mg}, 58 \%$ yield). UPLC $t r=1.41 \mathrm{~min}$ (Instrument A); HRMS (ESI) $\mathrm{m} / z$ calcd for C23H28INO4S2 [M+H] ${ }^{+}$574.0577, found 574.0590; ${ }^{1} \mathrm{H}$ NMR (400 MHz, CHLOROFORM-d) $\delta 7.57(\mathrm{~d}, J=8.0 \mathrm{~Hz}, 1 \mathrm{H}), 7.44-7.29(\mathrm{~m}, 3 \mathrm{H}), 7.25$ $7.18(\mathrm{~m}, 1 \mathrm{H}), 6.89(\mathrm{~d}, J=9.0 \mathrm{~Hz}, 2 \mathrm{H}), 4.42(\mathrm{dd}, J=6.9,4.2 \mathrm{~Hz}, 1 \mathrm{H}), 3.91-3.78(\mathrm{~m}, 4 \mathrm{H}), 2.92(\mathrm{dt}$, $J=12.7,8.6 \mathrm{~Hz}, 1 \mathrm{H}), 2.69-2.59(\mathrm{~m}, 2 \mathrm{H}), 2.47-2.30(\mathrm{~m}, 1 \mathrm{H}), 2.23$ (dt, $J=16.8,5.6 \mathrm{~Hz}, 1 \mathrm{H}), 1.95$ (ddt, $J=13.5,9.0,4.5 \mathrm{~Hz}, 1 \mathrm{H}), 1.83-1.58(\mathrm{~m}, 1 \mathrm{H}), 1.16(\mathrm{~s}, 9 \mathrm{H}) ;{ }^{13} \mathrm{C} \mathrm{NMR}(101 \mathrm{MHz}$, CHLOROFORM-d) $\delta 164.1,142.2,137.2,135.4,132.4,131.9,130.4$ (2C), 127.2 (2C), 114.0, $94.9,73.2,63.9,57.5,55.7,40.0,36.6,26.4,24.8,23.5(3 \mathrm{C}) ;[\alpha]_{\mathrm{D}}^{20}=9.6(c=2.50$ in $\mathrm{CHCl} 3)$.

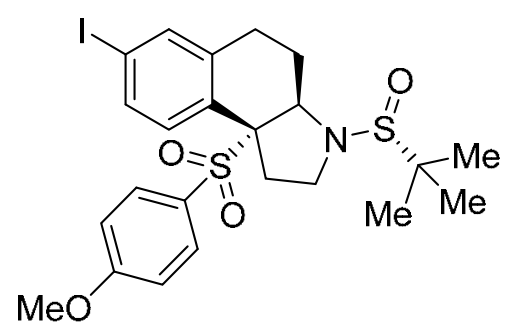

(3aR,9bR)-3-((R)-tert-butylsulfinyl)-7-iodo-9b-((4methoxyphenyl)sulfonyl)-2,3,3a,4,5,9b-hexahydro-1Hbenzo[e]indole (7c). Compound was synthesized according to General Procedure B. The product was purified using an ISCO teledyne instrument with a $12 \mathrm{~g}$ silica gel column while eluting with hexanes and EtOAc (gradient of 0 to $100 \%$ EtOAc) and was isolated as a white solid (116 mg, 52\% yield). UPLC $t r=1.41$ min (Instrument A); HRMS (ESI) $m / z$ calcd for C23H28INO3S2 [M+H] $]^{+} 558.0628$, found 558.0639; ${ }^{1} \mathrm{H}$ NMR (400 MHz, CHLOROFORM-d) $\delta 7.63$ (d, $\left.J=8.6 \mathrm{~Hz}, 1 \mathrm{H}\right), 7.60$ (s, 1H), 7.51 $(\mathrm{d}, J=8.4 \mathrm{~Hz}, 1 \mathrm{H}), 7.38$ - $7.20(\mathrm{~m}, 5 \mathrm{H}), 4.61(\mathrm{dd}, J=6.4,3.9 \mathrm{~Hz}, 1 \mathrm{H}), 4.09$ - $4.04(\mathrm{~m}, 1 \mathrm{H}), 3.97$ $3.85(\mathrm{~m}, 2 \mathrm{H}), 2.97-2.83(\mathrm{~m}, 2 \mathrm{H}), 2.78-2.61(\mathrm{~m}, 2 \mathrm{H}), 2.46-2.39(\mathrm{~m}, 1 \mathrm{H}), 2.12-2.01(\mathrm{~m}, 1 \mathrm{H})$, 1.93 - 1.77 (m, 1H), 1.19 (s, 9H); ${ }^{13} \mathrm{C}$ NMR (101 MHz, CHLOROFORM-d) $\delta$ 141.6, 137.9, 
136.2, 131.5 (2C), 131.3, 129.9, 128.9 (2C), 128.7, 126.2, 95.5, 73.3, 63.8, 57.6, 56.2, 39.7, 36.9, $26.3,24.8,23.5(3 \mathrm{C}) ;[\alpha]_{\mathrm{D}}^{20}=-55(c=2.50$ in $\mathrm{CHCl} 3)$.<smiles>CC(C)(C)S(=O)N1CC[C@]2(S(=O)(=O)Cc3ccccc3)c3ccc(I)cc3CC[C@H]12</smiles>

(3aR,9bR)-3-((R)-tert-butylsulfinyl)-9b-((4fluorophenyl)sulfonyl)-7-(perfluoropropan-2-yl)-2,3,3a,4,5,9bhexahydro-1H-benzo[e]indole (7d). Compound was synthesized according to General Procedure B. The product was purified using an ISCO teledyne instrument with a $12 \mathrm{~g}$ silica gel column while

eluting with hexanes and EtOAc (gradient of 0 to $100 \%$ EtOAc) and was isolated as a white solid (153 $\mathrm{mg}, 64 \%$ yield). UPLC $t r=1.58 \mathrm{~min}$ (Instrument A); HRMS (ESI) $\mathrm{m} / z$ calcd for C25H25F8NO3S2 [M+H] ${ }^{+} 603.1148$, found 603.1150; ${ }^{1} \mathrm{H}$ NMR (400 MHz, CHLOROFORM-d) $\delta 7.76(\mathrm{~d}, J=8.5 \mathrm{~Hz}, 1 \mathrm{H}), 7.52$ (br d, $J=8.4 \mathrm{~Hz}, 1 \mathrm{H}), 7.35$ (dd, $J=8.7,5.1 \mathrm{~Hz}, 2 \mathrm{H}), 7.30$ - 7.25 (m, $1 \mathrm{H}), 7.02(\mathrm{t}, J=8.5 \mathrm{~Hz}, 2 \mathrm{H}), 4.49$ (dd, $J=8.1,4.7 \mathrm{~Hz}, 1 \mathrm{H}), 3.97$ (ddd, $J=10.6,8.0,4.4 \mathrm{~Hz}, 1 \mathrm{H})$, $3.13(\mathrm{dt}, J=13.0,8.1 \mathrm{~Hz}, 1 \mathrm{H}), 2.82-2.63(\mathrm{~m}, 2 \mathrm{H}), 2.54-2.47$ (m, 1H), 2.14 (ddd, $J=16.2,8.5$, $4.2 \mathrm{~Hz}, 1 \mathrm{H}), 2.01-1.92(\mathrm{~m}, 1 \mathrm{H}), 1.69-1.54(\mathrm{~m}, 1 \mathrm{H}), 1.20(\mathrm{~s}, 9 \mathrm{H}) ;{ }^{13} \mathrm{C}$ NMR $(101 \mathrm{MHz}$, CHLOROFORM-d) $\delta 141.6,137.9,136.2,131.5,131.3(2 \mathrm{C}), 129.9,128.9,128.8,128.7$ (2C), $126.2,95.5,73.3,63.8,57.6,56.2,43.5,39.7,36.9,26.3,24.8,23.5(3 \mathrm{C}) ;[\alpha]_{\mathrm{D}}{ }^{20}=-55(c=2.50$ in $\mathrm{CHCl3}$ ).



\section{(3aR,9bR)-3-((R)-tert-butylsulfinyl)-9b-((4-} fluorophenyl)sulfonyl)-2,3,3a,4,5,9b-hexahydro-1Hbenzo[e]indole (7e). Compound was synthesized according to General Procedure B. The product was purified using an ISCO teledyne instrument with a $12 \mathrm{~g}$ silica gel column while eluting with hexanes and EtOAc (gradient of 0 to $100 \%$ EtOAc) and was isolated as a white solid (153 $\mathrm{mg}, 64 \%$ yield). UPLC $t r=$ 1.60 min (Instrument A); HRMS (ESI) $\mathrm{m} / \mathrm{z}$ calcd for C25H25F8NO3S2 $[\mathrm{M}+\mathrm{H}]^{+}$604.1221, found 604.1198; ${ }^{1} \mathrm{H}$ NMR (CHLOROFORM-d, 400MHz): $\delta=7.74(\mathrm{~d}, J=8.5 \mathrm{~Hz}, 1 \mathrm{H}), 7.50(\mathrm{br} \mathrm{d}, J=8.5 \mathrm{~Hz}, 1 \mathrm{H}), 7.31-7.36(\mathrm{~m}, 2 \mathrm{H}), 7.24(\mathrm{~s}, 1 \mathrm{H}), 6.97-7.03$ (m, 2H), 4.47 (dd, $J=8.2,4.8 \mathrm{~Hz}, 1 \mathrm{H}), 3.95$ (ddd, $J=10.6,8.1,4.4 \mathrm{~Hz}, 1 \mathrm{H}), 3.06-3.16$ (m, $1 \mathrm{H})$, 2.72-2.80 (m, 1H), 2.62-2.71 (m, 1H), 2.45-2.53 (m, 1H), 2.07-2.16 (m, 1H), 1.91-2.00 (m, 1H), 1.56-1.68 (m, 1H), 1.18 ppm (s, 9H); ${ }^{13} \mathrm{C}$ NMR (CHLOROFORM-d, 176MHz): $\delta=166.0(\mathrm{~d}$, $J=257 \mathrm{~Hz}, 1 \mathrm{C}), 141.0$ (d, $J=1.8 \mathrm{~Hz}, 1 \mathrm{C}), 134.2,132.9$ (d, $J=8.8 \mathrm{~Hz}, 2 \mathrm{C}), 131.7$ (d, $J=3.5 \mathrm{~Hz}, 1 \mathrm{C})$, 131.0 (d, $J=1.8 \mathrm{~Hz}, 1 \mathrm{C}), 127.1$ (d, $J=21 \mathrm{~Hz}, 1 \mathrm{C}), 125.4$ (d, $J=11 \mathrm{~Hz}, 1 \mathrm{C}), 123.6$ (d, $J=8.8 \mathrm{~Hz}$, 1C), 120.4 (dq, $J=287,28 \mathrm{~Hz}, 2 \mathrm{C}), 115.9$ (d, $J=21 \mathrm{~Hz}, 2 \mathrm{C}), 91.1$ (m, 1C), 74.0, 64.5, 57.5, 40.3, $36.2,27.5,25.8,23.4(3 \mathrm{C}) \mathrm{ppm} ;[\alpha]_{\mathrm{D}}{ }^{20}=-24(c=0.286$ in $\mathrm{AcCN})$.



(3aR,9bR)-3-((R)-tert-butylsulfinyl)-9b-((4fluorophenyl)sulfonyl)-2,3,3a,4,5,9b-hexahydro-1Hbenzo[e]indole (7f). Compound was synthesized according to General Procedure B. The product was purified using an ISCO teledyne instrument with a $12 \mathrm{~g}$ silica gel column while eluting with hexanes and EtOAc (gradient of 0 to $100 \%$ EtOAc) and was isolated as a white solid (119 mg, 68\% yield). UPLC $t r=1.58 \mathrm{~min}$

(Instrument A); HRMS (ESI) $\mathrm{m} / z$ calcd for C22H26NO3FS2 [M+H] $]^{+} 436.1411$, found 436.1424; ${ }^{1} \mathrm{H}$ NMR (400 MHz, CHLOROFORM-d) $\delta 7.56$ (d, $\left.J=6.8 \mathrm{~Hz}, 1 \mathrm{H}\right), 7.43$ - 7.37 (m, 2H), 7.31 - 
$7.24(\mathrm{~m}, 2 \mathrm{H}), 7.08-7.00(\mathrm{~m}, 3 \mathrm{H}), 4.48$ (dd, $J=7.9,4.6 \mathrm{~Hz}, 1 \mathrm{H}), 3.94$ (ddd, $J=10.5,8.1,4.1 \mathrm{~Hz}$, $1 \mathrm{H}), 3.06$ (dt, $J=12.8,8.3 \mathrm{~Hz}, 1 \mathrm{H}), 2.76-2.60(\mathrm{~m}, 2 \mathrm{H}), 2.52-2.43(\mathrm{~m}, 1 \mathrm{H}), 2.17-2.06(\mathrm{~m}, 1 \mathrm{H})$, $1.97-1.88(\mathrm{~m}, 1 \mathrm{H}), 1.42-1.22(\mathrm{~m}, 1 \mathrm{H}), 1.18(\mathrm{~s}, 9 \mathrm{H}) ;{ }^{13} \mathrm{C}$ NMR (101 MHz, CHLOROFORM-d) $\delta 167.0$ (d, $J=250 \mathrm{~Hz}, 1 \mathrm{C}), 140.1,133.0$ (d, $J=9 \mathrm{~Hz}, 2 \mathrm{C}), 132.1$ (d, $J=4 \mathrm{~Hz}, 1 \mathrm{C}), 130.5,130.3$, 128.7, 128.3, 126.5, 115.8 (d, $J=20 \mathrm{~Hz}, 2 \mathrm{C}), 74.1,64.6,57.5,40.4,36.5,27.5,25.5,23.5$ (3C); ${ }^{19} \mathrm{~F}$ NMR (375 MHz, CHLOROFORM-d) $\delta 103.0[\alpha] \mathrm{D}^{20}=-45(c=2.65$ in $\mathrm{CHCl} 3)$.

\section{Summary:}

The crystal structure of $\mathbf{7 f}$ was characterized by single crystal X-ray diffraction. The X-ray data were collected using a colorless block shaped crystal grown from $\mathrm{MeOH}$. There is one crystallographically independent molecule per asymmetric unit. The stereochemistry of the pyrrolidine and sulfinyl sulfur was determined to be (3aR,9bR)-3-((R)-tertbutylsulfinyl), respectively, based on the refinement of anomalous scattering.

\section{Structure by X-ray crystallography:}

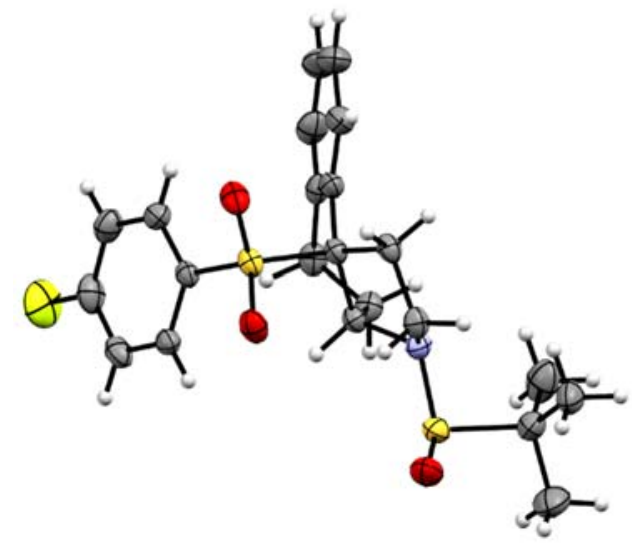

\section{Crystal Data:}

Chemical formula: $\mathrm{C}_{22} \mathrm{H}_{26} \mathrm{FNO}_{3} \mathrm{~S}_{2}$

$\mathrm{MW}=435.56$

Crystal system: Orthorhombic

Space Group: $\mathrm{P} 2{ }_{1} 2_{1} 2_{1}$

$a=7.3824(6) \AA \quad \alpha=90^{\circ}$

$b=11.4088(9) \AA \quad \beta=90^{\circ}$

$c=25.182(2) \AA \quad \gamma=90^{\circ}$

$V=2121.0(3) \AA^{3}$

No. of molecules/cell: $Z=4$

No. of unique molecules per asymmetric unit: $Z^{\prime}=1$

Calculated crystal density: $\mathrm{d}_{\mathrm{x}}=1.364 \mathrm{~g} \mathrm{~cm}^{-3}$

\section{Experimental:}

\section{Crystallization}

Crystal source: $\mathrm{MeOH}$

Crystal description: colorless block

Crystal size $\left(\mathrm{mm}^{3}\right): 0.30 \times 0.25 \times 0.25$

Data Collection

Temperature (K): 200

Instrument: Bruker APEX Kappa with Microstar H 


\section{Refinement:}

Final R [I>2sigma(I)]: R1 $=0.0262, \mathrm{wR} 2=0.0688$

Final R (all): R1 $=0.0271, \mathrm{wR} 2=0.0693$

Goodness-of-Fit: 1.060

Flack $(\mathrm{x})=0.004(5)$

P3true $=1.000$

$\operatorname{Hooft}(\mathrm{y})=0.001(4)$

Treatment of Hydrogen Atoms:

All hydrogen atom positions were calculated using idealized geometry with standard bond lengths and angles during structure refinement. They were assigned isotropic temperature factors and were included in structure factor calculations with fixed parameters.<smiles>COc1ccc2c(c1)CC[C@H]1N(S(=O)C(C)(C)C)CC[C@@]21S(=O)(=O)c1ccc(F)cc1</smiles>

((3aR,9bR)-3-((R)-tert-butylsulfinyl)-9b-((4fluorophenyl)sulfonyl)-7-methoxy-2,3,3a,4,5,9b-hexahydro1H-benzo[e]indole (7g). Compound was synthesized according to General Procedure B. The product was purified using an ISCO teledyne instrument with a $12 \mathrm{~g}$ silica gel column while eluting with hexanes and EtOAc (gradient of 0 to $100 \%$ EtOAc) and was isolated as a white solid ( $137 \mathrm{mg}, 74 \%$ yield). UPLC $t r=1.58$ min (Instrument A); HRMS (ESI) $\mathrm{m} / z$ calcd for C23H28NO4FS2 $[\mathrm{M}+\mathrm{H}]^{+} 466.1517$, found 466.1526; ${ }^{1} \mathrm{H}$ NMR (400 MHz, CHLOROFORM-d) $\delta 7.48-7.28(\mathrm{~m}, 3 \mathrm{H}), 7.07$ (t, $J=8.1 \mathrm{~Hz}$, $2 \mathrm{H}), 6.81(\mathrm{dd}, J=8.8,2.7 \mathrm{~Hz}, 1 \mathrm{H}), 6.56(\mathrm{~d}, J=2.7 \mathrm{~Hz}, 1 \mathrm{H}), 4.44(\mathrm{dd}, J=7.5,4.5 \mathrm{~Hz}, 1 \mathrm{H}), 3.91$ (ddd, $J=10.5,8.0,3.7 \mathrm{~Hz}, 1 \mathrm{H}), 3.84-3.79(\mathrm{~m}, 3 \mathrm{H}), 2.99(\mathrm{dt}, J=12.7,8.4 \mathrm{~Hz}, 1 \mathrm{H}), 2.73-2.57$ (m, 2H), 2.43 (ddd, $J=12.6,6.0,3.8 \mathrm{~Hz}, 1 \mathrm{H}), 2.25$ - 1.99 (m, 1H), 1.97 - 1.70 (m, 1H), 1.70 - 1.56 (m, 1H), $1.17(\mathrm{~s}, 9 \mathrm{H}) ;{ }^{13} \mathrm{C}$ NMR (101 MHz, CHLOROFORM-d) $\delta 166.0$ (d, $\left.J=250 \mathrm{~Hz}, 1 \mathrm{C}\right)$, $159.7,141.6,133.1$ (d, $J=9 \mathrm{~Hz}, 1 \mathrm{C}), 132.3,131.6,122.1,115.9$ (d, $J=22 \mathrm{~Hz}, 2 \mathrm{C}), 112.8$ (d, $J=43$ $\mathrm{Hz}, 2 \mathrm{C}), 73.6,64.4,57.5,55.3,41.0,40.3,36.6,27.2,25.7,23.5(3 \mathrm{C}) ;{ }^{19} \mathrm{~F}$ NMR (375 MHz, CHLOROFORM-d) $\delta 103.0[\alpha]_{\mathrm{D}^{20}}=-12(c=2.85$ in $\mathrm{CHCl} 3)$.

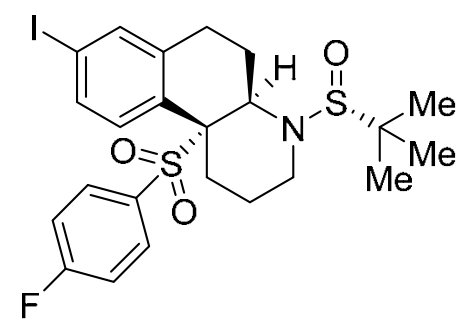

(4aR,10bR)-4-((R)-tert-butylsulfinyl)-10b-((4fluorophenyl)sulfonyl)-8-iodo-1,2,3,4,4a,5,6,10boctahydrobenzo[f]quinoline. (7h) Compound was synthesized according to the General Procedure B using annulating reagent $\mathbf{4 h}$ instead of 4e. The product was purified by flash column chromatography using hexanes-EtOAc (gradient from $0 \%$ to $100 \%$ EtOAc) as the mobile phase using a Combiflash instrument and was isolated as a white solid (78 $\mathrm{mg}, 35 \%$ yield). UPLC $t r=1.45 \mathrm{~min}$ (Instrument A); HRMS (ESI) $\mathrm{m} / \mathrm{z}$ calcd for $\mathrm{C} 23 \mathrm{H} 27 \mathrm{FINO} 3 \mathrm{~S} 2[\mathrm{M}+\mathrm{H}]+{ }^{+}$576.0534, found $576.0543 ;{ }^{1} \mathrm{H}$ NMR $(400 \mathrm{MHz}$, CHLOROFORM-d) $\delta$ 7.40-7.49 (m, 4H), $7.10(\mathrm{t}, J=8.54 \mathrm{~Hz}, 2 \mathrm{H}), 6.73-6.78(\mathrm{~m}, 1 \mathrm{H}), 4.37-4.43$ (m, $1 \mathrm{H}), 3.45-3.54(\mathrm{~m}, 1 \mathrm{H}), 2.86-2.94(\mathrm{~m}, 1 \mathrm{H}), 2.50-2.66(\mathrm{~m}, 2 \mathrm{H}), 2.42(\mathrm{td}, J=13.32,3.51 \mathrm{~Hz}$, $1 \mathrm{H}), 2.20-2.32(\mathrm{~m}, 2 \mathrm{H}), 1.87-1.99(\mathrm{~m}, 1 \mathrm{H}), 1.73-1.83(\mathrm{~m}, 1 \mathrm{H}), 1.59-1.73(\mathrm{~m}, 1 \mathrm{H}), 1.15(\mathrm{~s}, 9 \mathrm{H})$; ${ }^{13} \mathrm{C}$ NMR (CHLOROFORM-d, 101MHz) $\delta 166.1$ (d, $\left.J=256 \mathrm{~Hz}, 1 \mathrm{C}\right), 144.4,137.7,135.0,133.7$ (d, $J=9 \mathrm{~Hz}, 2 \mathrm{C}), 131.0,130.9$ (d, $J=3 \mathrm{~Hz}, 1 \mathrm{C}), 129.8,115.9$ (d, $J=23 \mathrm{~Hz}, 2 \mathrm{C}), 95.0,70.9,59.5$, $58.3,36.0,27.3,27.2,27.1,23.8(3 \mathrm{C}), 18.3 \mathrm{ppm} ;[\alpha]_{\mathrm{D}}{ }^{20}=-14.18(c=2.51$ in DMSO $)$ 
<smiles>CC(C)(C)S(=O)N1CC[C@H](C(=O)c2ccccc2)[C@H]1c1ccccc1</smiles>

((2S,3S)-1-((R)-tert-butylsulfinyl)-2-phenylpyrrolidin-3yl)(phenyl)methanone (8a). Compound was synthesized according to General Procedure B. The product was purified using an ISCO teledyne instrument with a $12 \mathrm{~g}$ silica gel column while eluting with hexanes and EtOAc (gradient of 0 to $100 \%$ EtOAc) and was isolated as a white solid (42 $\mathrm{mg}, 30 \%$ yield). UPLC $t r=1.36 \mathrm{~min}$ (Instrument A); HRMS (ESI) $m / z$ calcd for $\mathrm{C} 21 \mathrm{H} 25 \mathrm{NO} 2 \mathrm{~S}[\mathrm{M}+\mathrm{H}]^{+} 356.1679$, found 356.1686; ${ }^{1} \mathrm{H}$ NMR (CHLOROFORM-d, 400MHz) $\delta 7.77$ (br d, $J=7.5 \mathrm{~Hz}, 2 \mathrm{H}$ ), 7.46-7.53 (m, 1H), 7.31-7.40 (m, 4H), 7.24-7.31 (m, 2H), 7.18-7.24 (m, 1H), 5.15 (d, J=8.3 Hz, 1H), 4.01$4.11(\mathrm{~m}, 1 \mathrm{H}), 3.95(\mathrm{dt}, J=10.2,7.9 \mathrm{~Hz}, 1 \mathrm{H}), 3.18(\mathrm{td}, J=10.0,6.5 \mathrm{~Hz}, 1 \mathrm{H}), 2.31-2.41(\mathrm{~m}, 1 \mathrm{H})$, 2.08-2.22 (m, 1H), $1.09(\mathrm{~s}, 9 \mathrm{H}) ;{ }^{13} \mathrm{C}$ NMR (CHLOROFORM-d, 101MHz) $\delta 198.1,141.8,136.4$, 133.2, 128.5 (2C), 128.4 (2C), 128.3 (2C), 127.7 (2C), 127.6, 70.4, 57.3, 55.6, 42.1, 32.4, 23.7 $(3 \mathrm{C}) ;[\alpha]_{\mathrm{D}}{ }^{20}=-27.99(c=3.00$ in $\mathrm{CHCl} 3)$.

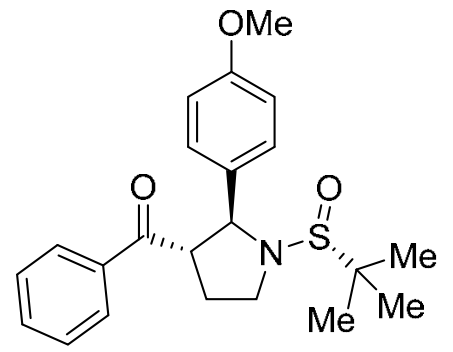

((2S,3S)-1-((R)-tert-butylsulfinyl)-2-(4methoxyphenyl)pyrrolidin-3-yl)(phenyl)methanone (8b). Compound was synthesized according to General Procedure B. The product was purified using an ISCO teledyne instrument with a $12 \mathrm{~g}$ silica gel column while eluting with hexanes and EtOAc (gradient of 0 to $100 \%$ EtOAc) and was isolated as a white solid (21 mg, 15\% yield). Data for major isomer, UPLC $t r=1.51 \mathrm{~min}$ (Instrument A); HRMS (ESI) $m / z$ calcd for $\mathrm{C} 22 \mathrm{H} 28 \mathrm{~N} 1 \mathrm{O} 3 \mathrm{~S} 1[\mathrm{M}+\mathrm{H}]^{+} 386.1790$,

found 386.1792; ${ }^{1} \mathrm{H}$ NMR (400 MHz, CHLOROFORM-d) $\delta 7.80$ (br d, $J=7.5 \mathrm{~Hz}, 2 \mathrm{H}$ ), 7.52 (br t, $J=7.4 \mathrm{~Hz}, 1 \mathrm{H}), 7.39$ (t, $J=7.7 \mathrm{~Hz}, 2 \mathrm{H}), 7.28$ (t, $J=4.2 \mathrm{~Hz}, 2 \mathrm{H}), 6.83$ (d, J=8.7 Hz, 2H), 5.09 (d, $J=8.6 \mathrm{~Hz}, 1 \mathrm{H}), 4.25-3.99(\mathrm{~m}, 1 \mathrm{H}), 3.98-3.88(\mathrm{~m}, 1 \mathrm{H}), 3.78(\mathrm{~s}, 3 \mathrm{H}), 3.23-3.08(\mathrm{~m}, 1 \mathrm{H}), 2.52$ $2.26(\mathrm{~m}, 1 \mathrm{H}), 2.26-2.09(\mathrm{~m}, 1 \mathrm{H}), 1.11(\mathrm{~m}, 9 \mathrm{H}) ;{ }^{13} \mathrm{C}$ NMR (101 MHz, CHLOROFORM-d) $\delta$ 198.3, 159.1, 136.5, 133.5, 133.2, 128.9 (2C), 128.6 (2C), 128.4 (2C), 113.8 (2C), 69.9, 57.3, $55.4,41.9,32.3,29.7,23.7(3 \mathrm{C}) ;[\alpha]_{\mathrm{D}^{20}}=-45.32(c=3.30$ in $\mathrm{CHCl} 3)$.<smiles>COc1ccc(C(=O)[C@@H]2CCN(S(=O)C(C)(C)C)[C@H]2c2ccccc2)cc1</smiles>

((2S,3S)-1-((R)-tert-butylsulfinyl)-2-phenylpyrrolidin-3yl)(4-methoxyphenyl)methanone (8c). Compound was synthesized according to General Procedure B. The product was purified using an ISCO teledyne instrument with a $12 \mathrm{~g}$ silica gel column while eluting with hexanes and EtOAc (gradient of 0 to $100 \%$ EtOAc) and was isolated as a white solid (14 mg, 10\% yield). Data for major isomer, UPLC $t r=$ $1.51 \mathrm{~min}$ (Instrument A); HRMS (ESI) $\mathrm{m} / z$ calcd for C22H28N1O3S1 [M+H] ${ }^{+} 386.1790$, found 386.1792; ${ }^{1} \mathrm{H}$ NMR (400 MHz, CHLOROFORM-d) $\delta 7.80$ (br d, $J=7.5 \mathrm{~Hz}, 2 \mathrm{H}$ ), 7.52 (br t, $J=7.4$ $\mathrm{Hz}, 1 \mathrm{H}), 7.39$ (t, $J=7.7 \mathrm{~Hz}, 2 \mathrm{H}), 7.28$ (t, $J=4.2 \mathrm{~Hz}, 2 \mathrm{H}), 6.83$ (d, $J=8.7 \mathrm{~Hz}, 2 \mathrm{H}), 5.09$ (d, $J=8.6$ $\mathrm{Hz}, 1 \mathrm{H}), 4.25$ - $3.99(\mathrm{~m}, 1 \mathrm{H}), 3.98-3.88(\mathrm{~m}, 1 \mathrm{H}), 3.78(\mathrm{~s}, 3 \mathrm{H}), 3.23$ - $3.08(\mathrm{~m}, 1 \mathrm{H}), 2.52$ - 2.26 $(\mathrm{m}, 1 \mathrm{H}), 2.26-2.09(\mathrm{~m}, 1 \mathrm{H}), 1.11(\mathrm{~m}, 9 \mathrm{H}) ;{ }^{13} \mathrm{C}$ NMR (101 MHz, CHLOROFORM-d) $\delta 198.3$, 159.1, 136.5, 133.5, 133.2, 128.9 (2C), 128.6 (2C), 128.4 (2C), 113.8 (2C), 69.9, 57.3, 55.4, $41.9,32.3,29.7,23.7(3 \mathrm{C}) ;[\alpha]_{\mathrm{D}}^{20}=-21.15(c=3.10$ in $\mathrm{CHCl} 3)$. 
<smiles>CC(C)(C)[S@](=O)N1CC[C@H](C(=O)c2ccccc2)[C@H]1c1ccc(C(F)(F)F)cc1</smiles>

((2S,3S)-1-((R)-tert-butylsulfinyl)-2-(4trofluorophenyl)pyrrolidin-3-yl)(phenyl)methanone (8d).

Compound was synthesized according to General Procedure B. The product was purified using an ISCO teledyne instrument with a $12 \mathrm{~g}$ silica gel column while eluting with hexanes and EtOAc (gradient of 0 to $100 \%$ EtOAc) and was isolated as a white solid (110 mg, 65\% yield). Data for major isomer, UPLC $t r=1.41 \mathrm{~min}$ (Instrument A); HRMS (ESI) $m / z$ calcd for $\mathrm{C} 22 \mathrm{H} 24 \mathrm{~F} 3 \mathrm{NO} 2 \mathrm{~S}[\mathrm{M}+\mathrm{H}]^{+} 424.1553$,

$7.46(\mathrm{~m}, 5 \mathrm{H}), 7.46-7.36(\mathrm{~m}, 2 \mathrm{H}), 5.28(\mathrm{br} \mathrm{d}, J=8.5 \mathrm{~Hz}, 1 \mathrm{H}), 4.11(\mathrm{br} \mathrm{t}, J=8.7 \mathrm{~Hz}, 1 \mathrm{H}), 3.98$ $3.85(\mathrm{~m}, 1 \mathrm{H}), 3.27-3.16(\mathrm{~m}, 1 \mathrm{H}), 2.49-2.35(\mathrm{~m}, 1 \mathrm{H}), 2.23-2.04(\mathrm{~m}, 1 \mathrm{H}), 1.13(\mathrm{~s}, 9 \mathrm{H}) ;{ }^{13} \mathrm{C}$ NMR (101 MHz, CHLOROFORM-d) $\delta$ 197.4, 146.0, 136.1, 133.5 (2C), 129.9 (q, J=33 Hz, 1C), 128.7 (2C), 128.3 (2C), 128.1 (2C), 125.5 (q, $J=3.7 \mathrm{~Hz}, 1 \mathrm{C}), 122.7,69.7,57.5,55.9,42.3,32.6$, $23.7(3 \mathrm{C}) ;{ }^{19} \mathrm{~F}$ NMR (376 MHz, CHLOROFORM-d) $\delta-62.54 ;[\alpha]_{\mathrm{D}}{ }^{20}=-630\left(c=5.0\right.$ in $\left.\mathrm{CHCl}_{3}\right)$.<smiles>CC(C)(C)S(=O)N1CC[C@H](C(=O)c2ccc(C(F)(F)F)cc2)[C@H]1c1ccc(F)cc1</smiles>

((2S,3S)-1-((R)-tert-butylsulfinyl)-2-(4fluorophenyl)pyrrolidin-3-yl)(4(trifluoromethyl)phenyl)methanone (8e). Compound was synthesized according to General Procedure B. The product was purified using an ISCO teledyne instrument with a $12 \mathrm{~g}$ silica gel column while eluting with hexanes and EtOAc (gradient of 0 to $100 \%$ EtOAc) and was isolated as a white solid (100 mg, $62 \%$ yield). Data for major isomer, UPLC $t r=1.42 \mathrm{~min}$ (Instrument A); HRMS (ESI) $m / z$ calcd for C22H23F4NO2S $[\mathrm{M}+\mathrm{H}]^{+}$442.1458, found 442.1464; ${ }^{1} \mathrm{H}$ NMR (400 MHz, CHLOROFORM-d) $\delta 7.91-7.86$ (m, $J=8.1 \mathrm{~Hz}, 2 \mathrm{H}), 7.70-7.65(\mathrm{~m}, J=8.2 \mathrm{~Hz}, 2 \mathrm{H}), 7.36-7.28(\mathrm{~m}, 2 \mathrm{H}), 7.00$ (t, $J=8.6 \mathrm{~Hz}, 2 \mathrm{H}), 5.13$ $(\mathrm{d}, J=8.5 \mathrm{~Hz}, 1 \mathrm{H}), 4.17-4.05(\mathrm{~m}, 1 \mathrm{H}), 3.94-3.87(\mathrm{~m}, 1 \mathrm{H}), 3.20(\mathrm{td}, J=10.0,6.5 \mathrm{~Hz}, 1 \mathrm{H}), 2.43$ $2.34(\mathrm{~m}, 1 \mathrm{H}), 2.23$ - $2.06(\mathrm{~m}, 1 \mathrm{H}), 1.20-1.10(\mathrm{~m}, 9 \mathrm{H}) ;{ }^{13} \mathrm{C}$ NMR (101 MHz, CHLOROFORMd) $\delta 197.2,162.3(\mathrm{~d}, J=235 \mathrm{~Hz}, 1 \mathrm{C}), 139.0,137.1(\mathrm{~d}, J=3.0 \mathrm{~Hz}, 2 \mathrm{C}), 134.6(\mathrm{q}, J=33 \mathrm{~Hz}, 1 \mathrm{C})$, 129.4 (d, $J=8.0 \mathrm{~Hz}, 2 \mathrm{C}$ ), 128.6 (2C), 125.7 (q, $J=3.7 \mathrm{~Hz}, 1 \mathrm{C}), 124.8,122.1,115.6,115.4,69.7$, 57.4, 56.0, 41.9, 32.1, $23.7(3 \mathrm{C}) ;[\alpha]_{\mathrm{D}}{ }^{20}=-17.4(c=2.4$ in $\mathrm{CHCl} 3)$.

\section{Summary:}

The crystal structure of $\mathbf{8 e}$ was characterized by single crystal X-ray diffraction. The X-ray data were collected using a colorless prism shaped crystal grown from $\mathrm{MeOH}-\mathrm{H}_{2} \mathrm{O}$ at $50^{\circ} \mathrm{C}$. There is one crystallographically independent molecule per asymmetric unit. The stereochemistry of the molecule was determined to be $2 S, 3 S$ and $R$ at sulfure based on the refinement of anomalous scattering. 


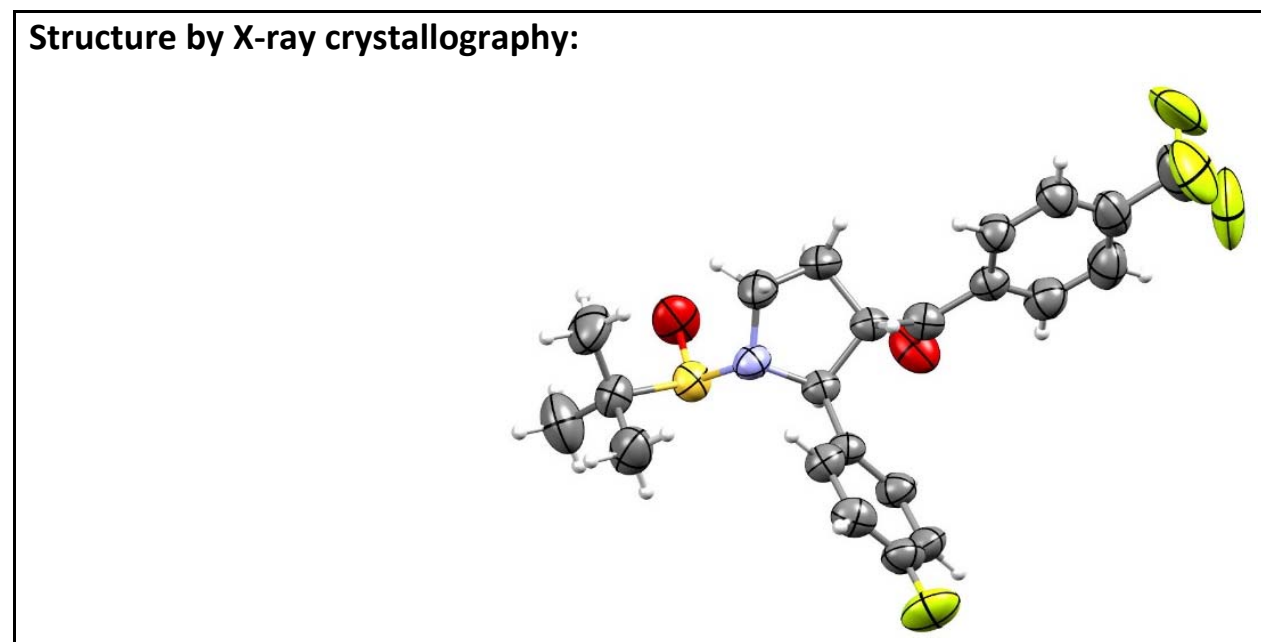

\section{Crystal Data:}

Chemical formula: $\mathrm{C}_{22} \mathrm{H}_{23} \mathrm{~F}_{4} \mathrm{NO}_{2} \mathrm{~S}$

$\mathrm{MW}=441.48$

Crystal system: Monoclinic

Space Group: $\mathrm{P} 2_{1}$

$a=13.3818(3) \AA \quad \alpha=90^{\circ}$

$b=5.8074(1) \AA \quad \beta=112.368(1)^{\circ}$

$c=15.1056(4) \AA \quad \gamma=90^{\circ}$

$V=1085.58(4) \AA^{3}$

Experimental:

Crystallization

Crystal source: $\mathrm{MeOH}-\mathrm{H}_{2} \mathrm{O}$

Crystal description: colorless plate

Crystal size $\left(\mathrm{mm}^{3}\right): 0.30 \times 0.25 \times 0.20$

$\underline{\text { Data Collection }}$

Temperature (K): 296

Instrument: Bruker APEX X8 Prospector with I $\mu \mathrm{S}$

No. of molecules/cell: $Z=2$

No. of unique molecules per asymmetric unit: $Z^{\prime}=1$

Calculated crystal density: $d_{x}=1.794 \mathrm{~g} \mathrm{~cm}^{-3}$

\section{Refinement:}

Final R [I>2sigma(I)]: R1 = 0.0770, wR2 $=0.1970$

Final R (all): R1 $=0.0787, \mathrm{wR} 2=0.1998$

Goodness-of-Fit: $1.071 \quad$ Flack $(x)=-0.02(4) \quad$ P3true $=1.000 \quad$ Hooft $(y)=-0.06(3)$

Treatment of Hydrogen Atoms:

All hydrogen atom positions were calculated using idealized geometry with standard bond lengths and angles during structure refinement. They were assigned isotropic temperature factors and were included in structure factor calculations with fixed parameters. 
<smiles>CC(C)(C)S(=O)N1CC[C@H](C(=O)c2ccccc2)[C@H]1C(F)(F)F</smiles>

((2S,3S)-1-((R)-tert-butylsulfinyl)-2-(trifluoromethyl)pyrrolidin3-yl)(phenyl)methanone (8f). Compound was synthesized according to General Procedure B. The product was purified using an ISCO teledyne instrument with a $12 \mathrm{~g}$ silica gel column while eluting with hexanes and EtOAc (gradient of 0 to $100 \%$ EtOAc) and was isolated as a white solid (111 mg, 80\% yield). Data for major isomer, UPLC $t r=1.29 \mathrm{~min}$ (Instrument A); HRMS (ESI) $\mathrm{m} / z$ calcd for $\mathrm{C} 16 \mathrm{H} 20 \mathrm{~F} 3 \mathrm{NO} 2 \mathrm{~S}[\mathrm{M}+\mathrm{H}]^{+} 348.1240$, found 348.1245; ${ }^{1} \mathrm{H}$ NMR (400 MHz, CHLOROFORM-d) $\delta 7.99(\mathrm{~d}, J=7.4 \mathrm{~Hz}, 2 \mathrm{H}), 7.62(\mathrm{t}, J=7.4 \mathrm{~Hz}, 1 \mathrm{H}), 7.51$ (t, $J=7.7 \mathrm{~Hz}, 2 \mathrm{H}), 4.98-4.89(\mathrm{~m}, 1 \mathrm{H}), 4.13-3.97(\mathrm{~m}, 2 \mathrm{H}), 3.01(\mathrm{td}, J=10.2,6.3 \mathrm{~Hz}, 1 \mathrm{H}), 2.45-$ $2.36(\mathrm{~m}, 1 \mathrm{H}), 1.96-1.82(\mathrm{~m}, 1 \mathrm{H}), 1.28-1.20(\mathrm{~m}, 9 \mathrm{H}) ;{ }^{13} \mathrm{C}$ NMR (101 MHz, CHLOROFORMd) $\delta 195.8,135.2,133.8,128.9$ (2C), 128.7 (2C), 125.9 (q, $J=280 \mathrm{~Hz}, 1 \mathrm{C}), 67.8$ (q, $J=30 \mathrm{~Hz}, 1 \mathrm{C}$ ), 58.7, 47.7, 43.3, 33.2, 23.3 (3C); ${ }^{19} \mathrm{~F}$ NMR (376 MHz, CHLOROFORM-d) $\delta$-74.44 (s, 3F); $[\alpha]_{\mathrm{D}}^{20}=-7.1(c=4.9$ in $\mathrm{CHCl} 3)$.

\section{X-ray structure of 8f:}

The crystal structure of $\mathbf{8 f}$ was characterized by single crystal X-ray diffraction. The X-ray data were collected using a colorless needle shaped crystal grown from $\mathrm{MeOH}-\mathrm{H}_{2} \mathrm{O}$ at $50^{\circ} \mathrm{C}$. There is one crystallographically independent molecule per asymmetric unit. The stereochemistry of the pyrrolidine and sulfur was determined to be $2 S, 3 S$ and $R$, respectively, based on the refinement of anomalous scattering.

\section{Structure by X-ray crystallography:}

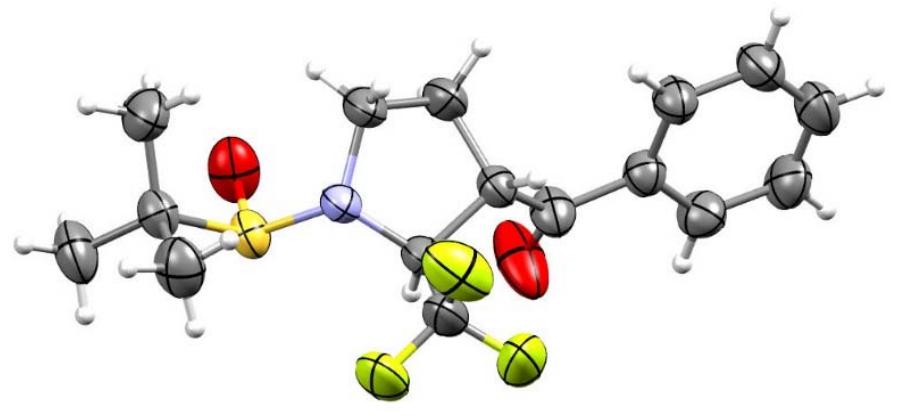




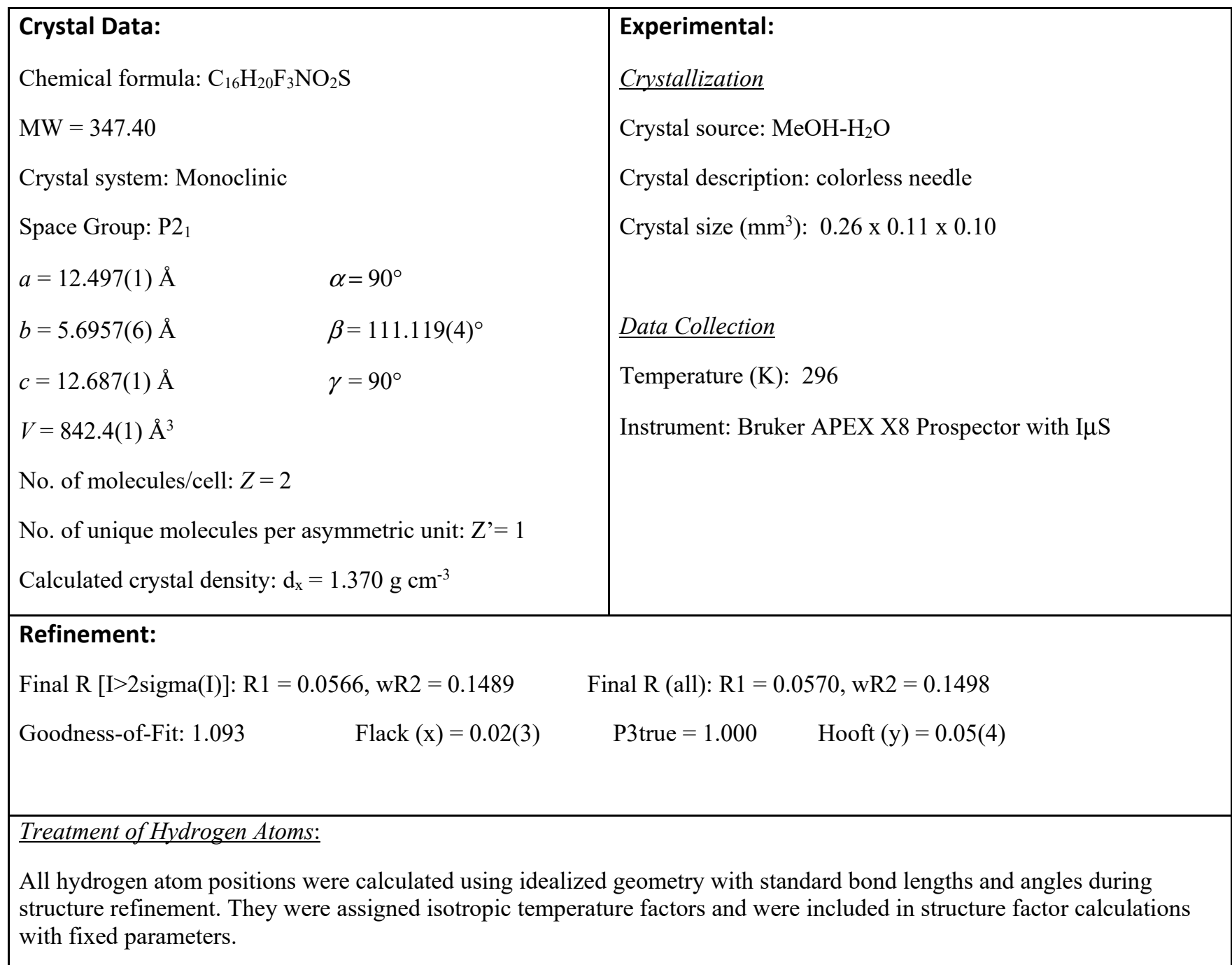<smiles>COC(=O)[C@H]1CCN(S(=O)C(C)(C)C)[C@@H]1C(F)(F)F</smiles>

Methyl (2S,3S)-1-((R)-tert-butylsulfinyl)-2(trifluoromethyl)pyrrolidine-3-carboxylate (8g). Compound was synthesized according to General Procedure B. The product was purified using an ISCO teledyne instrument with a $12 \mathrm{~g}$ silica gel column while eluting with hexanes and EtOAc (gradient of 0 to $100 \%$ EtOAc) and was isolated as a white solid (100 $\mathrm{mg}, 84 \%$ yield). Data for major isomer, UPLC $\mathrm{tr}$ $=1.11 \mathrm{~min}$ (Instrument A); HRMS (ESI) $\mathrm{m} / z$ calcd for $\mathrm{C} 11 \mathrm{H} 18 \mathrm{~F} 3 \mathrm{NO} 3 \mathrm{~S}[\mathrm{M}+\mathrm{H}]^{+} 302.1032$, found 302.1034; ${ }^{1} \mathrm{H}$ NMR (400 MHz, CHLOROFORM-d) $\delta 4.65$ - 4.57 (m, 1H), 3.98 (ddd, $J=11.0,7.8,3.6 \mathrm{~Hz}, 1 \mathrm{H}), 3.84-3.59$ (m, 3H), 3.17 (td, $J=8.2,5.6 \mathrm{~Hz}, 1 \mathrm{H}), 2.91$ (ddd, $J=10.8$, 9.3, $6.3 \mathrm{~Hz}, 1 \mathrm{H}), 2.33-2.20(\mathrm{~m}, 1 \mathrm{H}), 2.11-1.95(\mathrm{~m}, 1 \mathrm{H}), 1.29-1.18(\mathrm{~m}, 9 \mathrm{H}) ;{ }^{13} \mathrm{C} \mathrm{NMR}(101$ MHz, CHLOROFORM-d) $\delta 171.5,125.4$ (q, $J=280 \mathrm{~Hz}, 1 \mathrm{C}), 68.8$ (q, $J=31 \mathrm{~Hz}, 1 \mathrm{C}), 58.7,52.8$, 45.0, 42.8, 31.5, 23.3 (3C); ${ }^{19} \mathrm{~F}$ NMR (376 MHz, CHLOROFORM-d) $\delta-75.05$ (s, 3F); $[\alpha]_{\mathrm{D}}{ }^{20}=-$ $46(c=4.2$ in $\mathrm{CHCl} 3)$. 
<smiles>CCOC(=O)[C@H]1CCN(S(=O)C(C)(C)C)[C@@H]1C(F)(F)F</smiles>

\section{Ethyl (2S,3S)-1-((R)-tert-butylsulfinyl)-2-} (trifluoromethyl)pyrrolidine-3-carboxylate(8h). Compound was synthesized according to General Procedure B. The product was purified using an ISCO teledyne instrument with a $12 \mathrm{~g}$ silica gel column while eluting with hexanes and EtOAc (gradient of 0 to $100 \%$ 1.21 min (Instrument A); HRMS (ESI) $\mathrm{m} / z$ calcd for $\mathrm{C} 12 \mathrm{H} 20 \mathrm{~F} 3 \mathrm{NO} 3 \mathrm{~S}[\mathrm{M}+\mathrm{H}]^{+} 316.1189$, found 316.1190; ${ }^{1} \mathrm{H}$ NMR (400 MHz, CHLOROFORM-d) $\delta 4.64-4.56$ (m, 1H), 4.23 (q, $J=7.2 \mathrm{~Hz}$, 2H), 3.98 (ddd, $J=11.0,7.9,3.5 \mathrm{~Hz}, 1 \mathrm{H}$ ), 3.15 (td, $J=8.2,5.7 \mathrm{~Hz}, 1 \mathrm{H}$ ), 2.91 (ddd, $J=10.8,9.3,6.4$ $\mathrm{Hz}, 1 \mathrm{H}), 2.33-2.23(\mathrm{~m}, 1 \mathrm{H}), 2.05(\mathrm{dq}, J=12.4,8.7 \mathrm{~Hz}, 1 \mathrm{H}), 1.38-1.20(\mathrm{~m}, 12 \mathrm{H}) ;{ }^{13} \mathrm{C}$ NMR (101 MHz, CHLOROFORM-d) $\delta 171.0,125.5$ (q, $J=279 \mathrm{~Hz}, 1 \mathrm{C}$ ), 68.8 (q, $J=31 \mathrm{~Hz}, 1 \mathrm{C}$ ), 61.8, 58.7, 45.2, 42.8, 31.5, 23.3 (3C), 14.1; ${ }^{19}$ F NMR (376 MHz, CHLOROFORM-d) $\delta$-74.99 (s, $3 \mathrm{~F}) ;[\alpha]_{\mathrm{D}^{20}}=-44(c=4.2$ in $\mathrm{CHCl} 3)$.

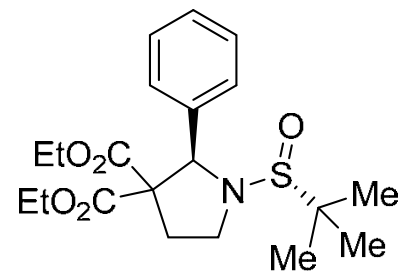

Diethyl (R)-1-((R)-tert-butylsulfinyl)-2-phenylpyrrolidine-3,3dicarboxylate (8ia). Compound was synthesized according to General Procedure B with the exception that after $2 \mathrm{~h}$ reaction time at $-78^{\circ} \mathrm{C}$, the reaction was allowed to warm slowly overnight. The product was purified using an ISCO teledyne instrument with a $12 \mathrm{~g}$ silica gel column while eluting with hexanes and EtOAc (gradient of 0 to $100 \%$

EtOAc) and the diastereoisomer mixture was isolated as a white solid (119 $\mathrm{mg}, 75 \%$ yield). The diastereoisomers were then separated for characterization using SFC. SFC Prep conditions: Column: Chiralpak OD, 30 x $250 \mathrm{~mm}, 5$ micron; Flow Rate: $100 \mathrm{~mL} / \mathrm{min}$; Oven Temperature: 40 C; BPR Setting: 120 bar; UV wavelength: $220 \mathrm{~nm}$; Mobile Phase: 90\% CO2 / 10\% IPA w/0.1\%DEA (isocratic); Injection: $300 \mathrm{uL}$ of $110.4 \mathrm{mg} / 3 \mathrm{~mL} \mathrm{MeOH}$ Data for major isomer 8ia, UPLC $t r=1.33$ min (Instrument A); HRMS (ESI) $\mathrm{m} / \mathrm{z}$ calcd for C20H29NO5S $[\mathrm{M}+\mathrm{H}]^{+}$396.1839, found 396.1847; ${ }^{1} \mathrm{H}$ NMR (CHLOROFORM-d, 400MHz) $\delta$ 7.21-7.30 (m, 5H), 5.55 (s, 1H), 4.19-4.30 (m, 2H), 3.78-3.90 (m, 2H), 3.58 (dq, J=10.7, 7.2 Hz, $1 \mathrm{H}$ ), 3.29 (ddd, $J=10.5,7.7,5.9 \mathrm{~Hz}, 1 \mathrm{H}$ ), 2.79 (dt, $J=13.2,7.3 \mathrm{~Hz}, 1 \mathrm{H}$ ), 2.33 (ddd, $J=13.4,8.0$, $5.9 \mathrm{~Hz}, 1 \mathrm{H}), 1.27$ (t, $J=7.1 \mathrm{~Hz}, 3 \mathrm{H}), 1.08(\mathrm{~s}, 9 \mathrm{H}), 0.88 \mathrm{ppm}(\mathrm{t}, J=7.1 \mathrm{~Hz}, 3 \mathrm{H}) ;{ }^{13} \mathrm{C}$ NMR (CHLOROFORM-d, 101MHz) $\delta$ 169.6, 168.3, 139.3, 128.1 (2C), 127.9 (2C), 127.8, 72.7, 65.4, $62.1,61.5,57.7,40.5,33.3,23.5(3 \mathrm{C}), 14.0,13.5 \mathrm{ppm} ;[\alpha]_{\mathrm{D}}^{20}=22(c=2.50$ in DMSO $)$.

Data for minor 8ib, HRMS (ESI) $\mathrm{m} / \mathrm{z}$ found $396.1845 ;{ }^{1} \mathrm{H}$ NMR (CHLOROFORM-d, 400MHz) $\delta 7.23-7.33(\mathrm{~m}, 2 \mathrm{H}), 5.54(\mathrm{~s}, 1 \mathrm{H}), 4.21-4.35(\mathrm{~m}, 1 \mathrm{H}), 4.08(\mathrm{ddd}, J=10.5,7.7,4.8 \mathrm{~Hz}, 1 \mathrm{H}), 3.73$ (dq, $J=10.7,7.2 \mathrm{~Hz}, 1 \mathrm{H}), 3.49-3.59$ (m, 1H), 3.24-3.34 (m, 1H), $2.70(\mathrm{dt}, J=13.3,7.9 \mathrm{~Hz}, 1 \mathrm{H})$, 2.34 (ddd, $J=13.2,6.5,4.8 \mathrm{~Hz}, 1 \mathrm{H}), 1.29(\mathrm{t}, J=7.1 \mathrm{~Hz}, 1 \mathrm{H}), 1.10$ (s, 3H), $0.92 \mathrm{ppm}(\mathrm{t}, J=7.2 \mathrm{~Hz}$, $1 \mathrm{H}) ;{ }^{13} \mathrm{C}$ NMR (CHLOROFORM-d, 101MHz) $\delta 169.9,168.0,138.9,128.5(2 \mathrm{C}), 128.1(2 \mathrm{C})$, $128.0,66.6,66.0,62.1,61.5,58.4,47.3,32.1,23.2(3 \mathrm{C}), 14.1,13.6 \mathrm{ppm} ;[\alpha] \mathrm{D}^{20}=-58(c=2.50$ in DMSO). 
Summary: Single crystal X-ray diffraction analysis has determined the structure and absolute configuration of 8ia. Crystals of the title compound grew as small needles from an aqueous acetone solution at room temperature. The crystal structure is neat (unsolvated/anhydrous) and belongs to the $P 2_{1}$ crystallographic space group with two symmetry independent conformers in the asymmetric unit $\left(Z^{\prime}=2\right)$. One conformer shows positional disorder of the ethyl ester side chain; disorder ratio refined to $~ 55: 45$. Analysis of the anomalous dispersion signal within the X-ray diffraction data show the enantiomer shown below as the correct stereochemical configuration; $\operatorname{Flack}(x)=0.02(3)$.

\section{Structure:}

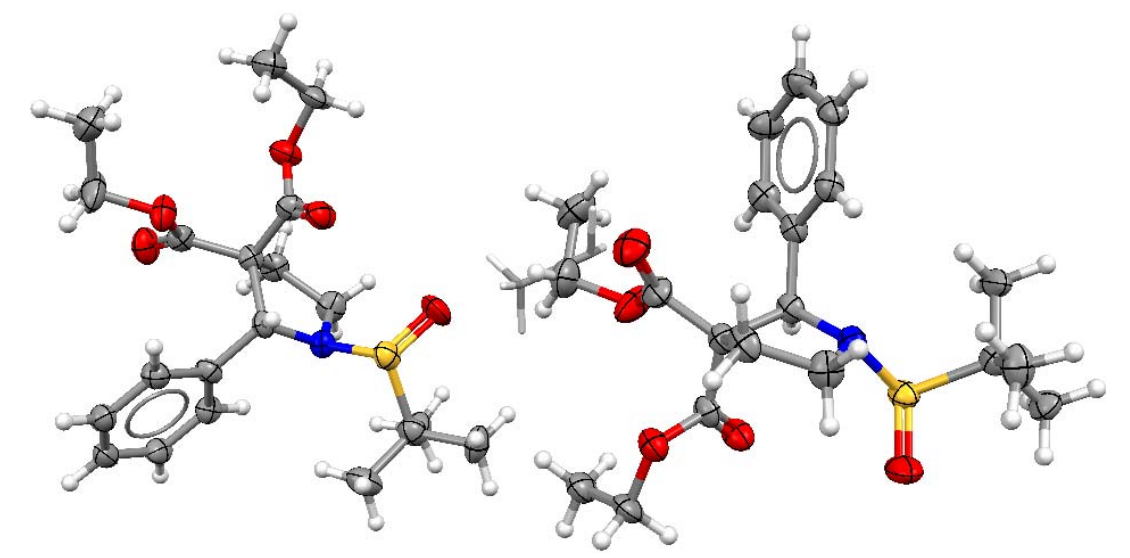

\section{Crystal Data:}

Chemical formula: $\mathrm{C}_{20} \mathrm{H}_{29} \mathrm{NO}_{5} \mathrm{~S}$

$F W=395.50$

Crystal system: Monoclinic

Space Group: $P 2_{1}$

$a=18.0493(12) \AA \quad \alpha=90^{\circ}$

$b=5.9821(5) \AA \quad \beta=113.270(4)^{\circ}$

$c=20.7805(17) \AA \quad \gamma=90^{\circ}$

$V=2061.2(3) \AA^{3}$

No. of molecules per cell $(Z)=4$

No. of molecules per asymmetric unit $\left(Z^{\prime}\right)=2$

Calculated crystal density $\left(\rho_{\text {calc }}\right)=1.274 \mathrm{~g} / \mathrm{cm}^{3}$

\section{Experimental:}

\section{Crystallization}

Crystal growth solvent(s): acetone/water

Crystal morphology: needle

Crystal size $\left(\mathrm{mm}^{3}\right): 0.27 \times 0.03 \times 0.015$

Data Collection

Temperature (K): 173.15

Instrument Type: Kappa2000 Goniometer, APEXII

Detector, MicrostarH Rotating Anode Genterator (Cu) 

Refinement:
Final R indices [l>2sigma(I)]: R1 $=0.0575, \mathrm{wR} 2=0.1314$
Final R indices [all data]: $\mathrm{R} 1=0.0916, \mathrm{WR} 2=0.1496$
Goodness-of-fit on $\mathrm{F}^{2}=1.035 \quad$ Flack $(\mathrm{x})=0.02(3)$
$\begin{aligned} & \text { Treatment of Hydrogen Atoms: All hydrogen atoms were calculated from an idealized geometry with } \\ & \text { standard bond lengths and angles and refined using a riding model. }\end{aligned}$<smiles>CCOC(C)(C)[C@]1(C)CCN(S(=O)C(C)(C)C)[C@@H]1c1ccccc1</smiles>

Ethyl (2R,3S)-1-((R)-tert-butylsulfinyl)-3-cyano-2phenylpyrrolidine-3-carboxylate (8j). Compound was synthesized according to General Procedure B with the exception that after $2 \mathrm{~h}$ reaction time at $-78{ }^{\circ} \mathrm{C}$, the reaction was allowed to warm slowly overnight. The product was purified using an ISCO teledyne instrument with a $12 \mathrm{~g}$ silica gel column while eluting with hexanes and EtOAc (gradient of 0 to $100 \%$ EtOAc) and was isolated as a white solid (90 mg, 65\% yield). Data for major isomer, UPLC $t r=1.22 \mathrm{~min}$ (Instrument A); HRMS (ESI) $\mathrm{m} / z$ calcd for C18H24N2O3S $[\mathrm{M}+\mathrm{H}]^{+}$349.1580, found 349.1586; ${ }^{1} \mathrm{H}$ NMR (CHLOROFORM-d, 400MHz) $\delta 7.32-7.45$ (m, $5 \mathrm{H}), 5.47(\mathrm{~s}, 1 \mathrm{H}), 4.33-4.42(\mathrm{~m}, 2 \mathrm{H}), 3.84-3.90(\mathrm{~m}, 2 \mathrm{H}), 2.72-2.80(\mathrm{~m}, 1 \mathrm{H}), 2.37$ (dt, $J=13.0,8.1$ $\mathrm{Hz}, 1 \mathrm{H}), 1.37-1.44(\mathrm{~m}, 3 \mathrm{H}), 0.99 \mathrm{ppm}(\mathrm{s}, 9 \mathrm{H}) ;{ }^{13} \mathrm{C}$ NMR (CHLOROFORM-d, 101MHz) $\delta 166.4$, $138.1,128.7,127.7,116.8,63.8,63.6,58.1,54.2,53.9,33.1,22.8,22.6,14.0 \mathrm{ppm} ;[\alpha]_{\mathrm{D}}{ }^{20}=40$ $(c=2.30$ in $\mathrm{CHCl3})$.

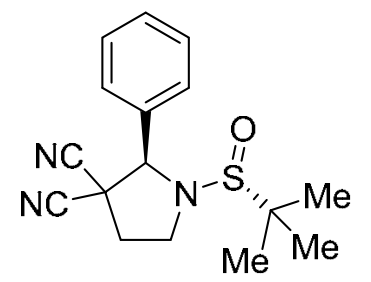

(R)-1-((R)-tert-butylsulfinyl)-2-phenylpyrrolidine-3,3-dicarbonitrile (8k). Compound was synthesized according to General Procedure B with the exception that after $2 \mathrm{~h}$ reaction time at $-78^{\circ} \mathrm{C}$, the reaction was allowed to warm slowly overnight. The product was purified using an ISCO teledyne instrument with a $12 \mathrm{~g}$ silica gel column while eluting with hexanes and EtOAc (gradient of 0 to $100 \%$ EtOAc) and was isolated as a white solid (74 mg, 62\% yield). Data for major isomer, UPLC $t r=1.11 \mathrm{~min}$ (Instrument A); HRMS (ESI) $m / z$ calcd for C16H19N3OS $[\mathrm{M}+\mathrm{H}]^{+} 302.1322$, found 302.1322; ${ }^{1} \mathrm{H}$ NMR (400 MHz, CHLOROFORM-d) $\delta 7.51$ - $7.28(\mathrm{~m}, 5 \mathrm{H}), 5.53(\mathrm{~s}, 1 \mathrm{H}), 4.05$ - $3.96(\mathrm{~m}, 1 \mathrm{H}), 3.93$ - 3.82 $(\mathrm{m}, 1 \mathrm{H}), 2.75-2.63(\mathrm{~m}, 2 \mathrm{H}), 1.07(\mathrm{~s}, 9 \mathrm{H}) ;{ }^{13} \mathrm{C}$ NMR (101 MHz, CHLOROFORM-d) $\delta 135.9$, 129.7, 129.2 (2C), 127.7 (2C), 114.3, 112.9, 65.1, 58.7, 51.9, 41.8, 36.8, $22.7(3 \mathrm{C}) ;[\alpha]_{\mathrm{D}}^{20}=109$ $(c=5.0$ in $\mathrm{CHCl} 3)$. Data for minor isomer, HRMS (ESI) $\mathrm{m} / z$ calcd for $\mathrm{C} 16 \mathrm{H} 20 \mathrm{~N} 3 \mathrm{OS}[\mathrm{M}+\mathrm{H}]^{+}$ 302.1322, found 302.1322; ${ }^{1} \mathrm{H}$ NMR (400 MHz, CHLOROFORM-d) $\delta 7.57$ - 7.38 (m, 5H), 5.01 (s, 1H), $4.32-4.11(\mathrm{~m}, 1 \mathrm{H}), 3.34$ (td, $J=10.6,6.4 \mathrm{~Hz}, 1 \mathrm{H}), 2.85$ (ddd, $J=12.9,6.4,2.0 \mathrm{~Hz}, 1 \mathrm{H})$, $2.72-2.59(\mathrm{~m}, 1 \mathrm{H}), 1.22(\mathrm{~s}, 9 \mathrm{H})$. 
<smiles>CC(C)(C)[S@@](=O)N1CC[C@](C#N)(C(=O)c2ccccc2)[C@H]1c1ccccc1</smiles>

(2R,3S)-3-benzoyl-1-((R)-tert-butylsulfinyl)-2-phenylpyrrolidine3-carbonitrile (81). Compound was synthesized according to General Procedure B with the exception that after $2 \mathrm{~h}$ reaction time at $-78^{\circ} \mathrm{C}$, the reaction was allowed to warm slowly overnight. The product was purified using an ISCO teledyne instrument with a $12 \mathrm{~g}$ silica gel column while eluting with hexanes and EtOAc (gradient of 0 to $100 \%$ EtOAc) and was isolated as a white solid (82 $\mathrm{mg}, 54 \%$ yield). Data for major isomer, UPLC $t r=1.28 \mathrm{~min}$ (Instrument A); HRMS (ESI) $m / z$ calcd for C22H24N2O2S [M+H] ${ }^{+}$381.1631, found 381.1639; ${ }^{1} \mathrm{H}$ NMR (CHLOROFORM-d, 400MHz) $\delta$ 8.26-8.31 (m, 2H), 7.64-7.70 (m, 1H), 7.40-7.60 (m, 7H), 3.93-4.00 (m, 1H), 3.79-3.88 (m, 1H), 2.96-3.04 (m, 1H), 2.60 (dt, $J=12.9,8.4 \mathrm{~Hz}, 1 \mathrm{H}), 0.97 \mathrm{ppm}(\mathrm{s}, 9 \mathrm{H}) ;{ }^{13} \mathrm{C}$ NMR (CHLOROFORMd, 101MHz) $\delta 189.9,138.7,134.2,133.3,129.7(2 \mathrm{C}), 129.0(2 \mathrm{C}), 128.9(2 \mathrm{C}), 127.8(2 \mathrm{C}), 118.5$, 64.0, 58.2, 56.8, 52.7, 52.7, 34.1, $22.6(3 \mathrm{C}) \mathrm{ppm} ;[\alpha]_{\mathrm{D}}{ }^{20}=-79(c=4.20$ in $\mathrm{CHCl} 3)$. Minor isomer wasn't isolated clean so no data is reported.

\section{Summary:}

The crystal structure of the minor isomer of $\mathbf{8 1}$ was characterized by single crystal $\mathrm{X}$-ray diffraction. The X-ray data were collected using a colorless needle shaped crystal grown from $\mathrm{MeOH}-\mathrm{H}_{2} \mathrm{O}$ at $50^{\circ} \mathrm{C}$. There are two crystallographically independent molecules per asymmetric unit. The stereochemistry of the pyrrolidine was determined to be $2 S, 3 R$ based on the refinement of anomalous scattering.

\section{Structure by X-ray crystallography:}

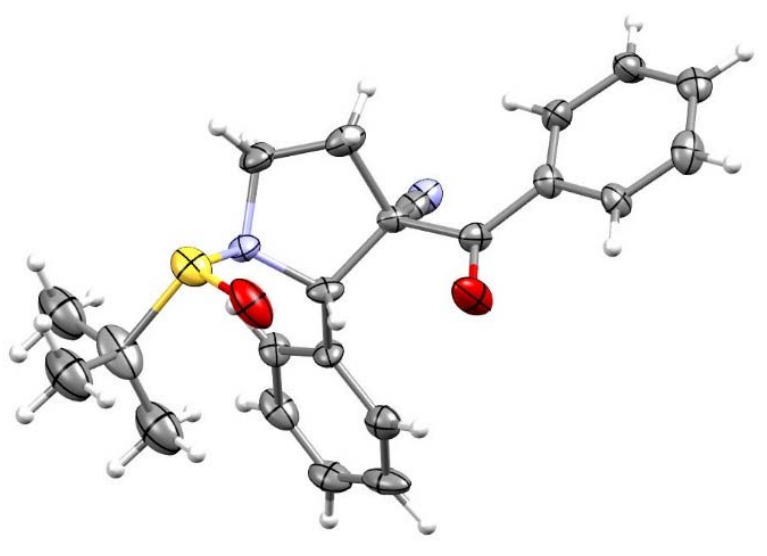




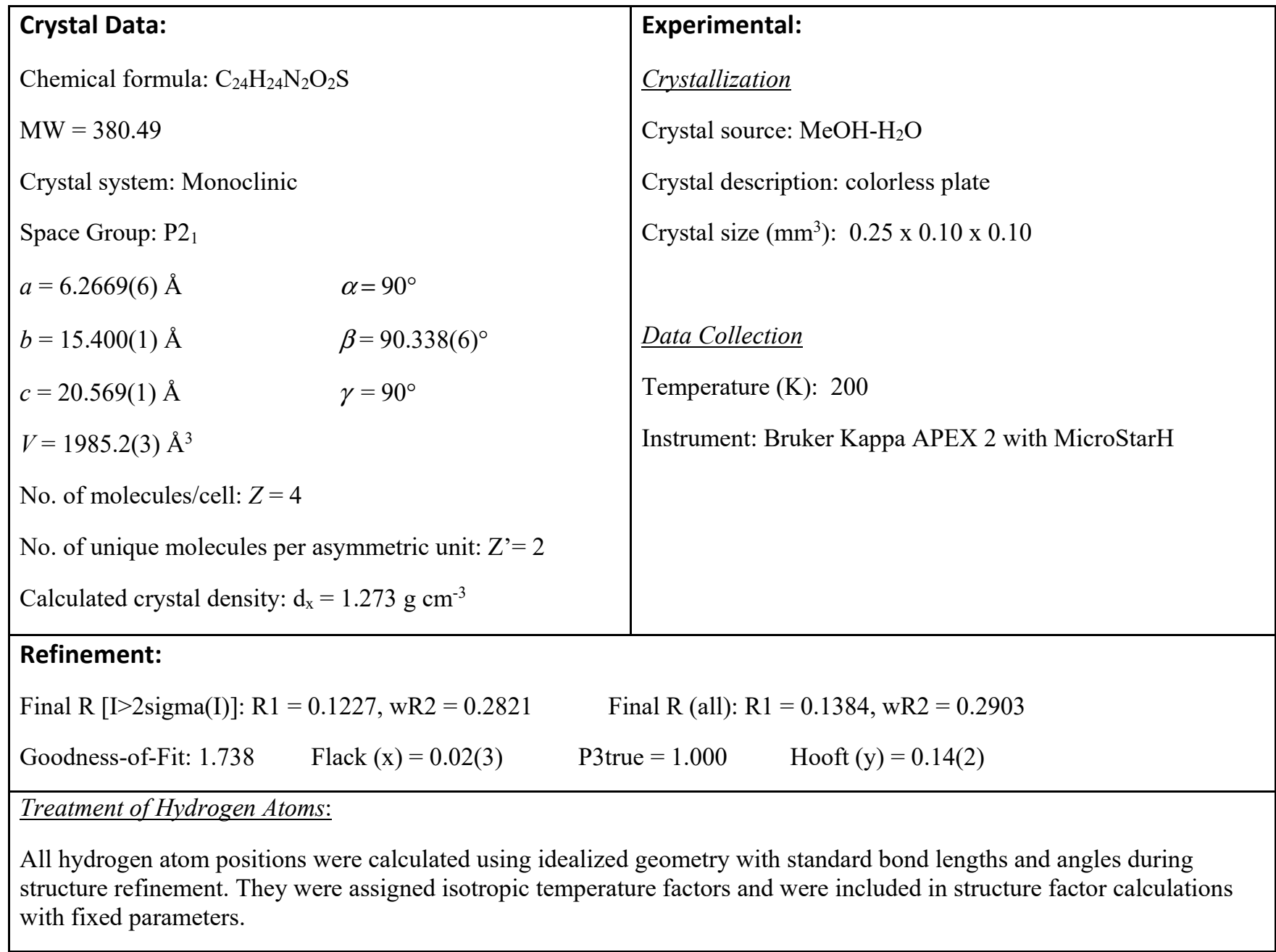<smiles>CCOC(=O)C1(C(=O)OCC)CCN(S(=O)c2c(C(C)C)cc(C(C)C)cc2C(C)C)[C@@H]1c1ccccc1</smiles>

\section{Diethyl (R)-2-phenyl-1-((R)-(2,4,6-} triisopropylphenyl)sulfinyl)pyrrolidine-3,3-dicarboxylate (8m). Compound was synthesized according to General Procedure B with the exception that after $2 \mathrm{~h}$ reaction time at $-78^{\circ} \mathrm{C}$, the reaction was allowed to warm slowly overnight. The product was purified using an ISCO teledyne instrument with a $12 \mathrm{~g}$ silica gel column while eluting with hexanes and EtOAc (gradient of 0 to $100 \%$ EtOAc) and was isolated as a white solid (120 mg, 68\% yield). UPLC $t r=$ $1.85 \mathrm{~min}$ (Instrument A); HRMS (ESI) $\mathrm{m} / z$ calcd for C31H43NO5S [M+Na] ${ }^{+}$564.2754, found 564.2763; ${ }^{1} \mathrm{H}$ NMR (CHLOROFORM-d, 400MHz) $\delta$ 7.24-7.37 (m, 5H), 7.00 (s, 2H), 5.61 (s, $1 \mathrm{H}), 4.19-4.39$ (m, 2H), 3.58-3.83 (m, 4H), 3.49 (dq, $J=10.7,7.2 \mathrm{~Hz}, 1 \mathrm{H}), 3.26$ (td, $J=8.2,1.6$ $\mathrm{Hz}, 1 \mathrm{H}), 2.80-2.95(\mathrm{~m}, 2 \mathrm{H}), 2.40-2.48(\mathrm{~m}, 1 \mathrm{H}), 1.20-1.30(\mathrm{~m}, 9 \mathrm{H}), 1.09(\mathrm{t}, J=6.6 \mathrm{~Hz}, 12 \mathrm{H}), 0.81$ ppm (t, $J=7.2 \mathrm{~Hz}, 3 \mathrm{H}) ;{ }^{13} \mathrm{C}$ NMR (CHLOROFORM-d, 101MHz) $\delta 170.0,168.2,151.6,149.4$, 138.2, 133.1, 129.1, 128.2, 127.8, 122.8, 67.8, 65.7, 62.2, 61.5, 42.6, 34.2, 32.5, 27.1, 24.5, 23.9, 23.8, 23.8, 14.0, $13.4 \mathrm{ppm} ; \alpha]_{\mathrm{D}^{20}}=14(c=0.36$ in $\mathrm{AcCN})$. 
<smiles>CCOC[C@@]1(C#N)CCN(S(=O)c2c(C(C)C)cc(C(C)C)cc2C(C)C)[C@H]1c1ccccc1</smiles>

\section{Ethyl (2R,3S)-3-cyano-2-phenyl-1-((R)-(2,4,6-} triisopropylphenyl)sulfinyl)pyrrolidine-3-carboxylate (8n). Compound was synthesized according to General Procedure B with the exception that after $2 \mathrm{~h}$ reaction time at $-78^{\circ} \mathrm{C}$, the reaction was allowed to warm slowly overnight. The product was purified using an ISCO teledyne instrument with a $12 \mathrm{~g}$ silica gel column while and was isolated as a white solid (150 $\mathrm{mg}, 61 \%$ yield). UPLC $t r=1.73 \mathrm{~min}$ (Instrument A); HRMS (ESI) $m / z$ calcd for C29H38N2O3S $[\mathrm{M}+\mathrm{H}]^{+} 495.2676$, found 495.2671; ${ }^{1} \mathrm{H}$ NMR (CHLOROFORM-d, 400MHz) $\delta$ 7.53-7.59 (m, 2H), 7.40-7.47 (m, 3H), $7.06(\mathrm{~s}, 2 \mathrm{H}), 4.95(\mathrm{~s}$, $1 \mathrm{H}), 4.25$ (dtt, $J=10.7,7.1,3.5 \mathrm{~Hz}, 2 \mathrm{H}), 3.81-3.99$ (m, 3H), 3.30 (ddd, $J=9.7,8.0,5.4 \mathrm{~Hz}, 1 \mathrm{H})$, 2.75-2.92 (m, 2H), 2.57 (ddd, $J=13.1,7.8,6.7 \mathrm{~Hz}, 1 \mathrm{H}), 1.13-1.28(\mathrm{~m}, 21 \mathrm{H}) ;{ }^{13} \mathrm{C}$ NMR

(CHLOROFORM-d, 101MHz) $\delta 166.4,152.3,149.7,133.5,131.7,129.9,129.2,128.5,123.1$, $117.5,70.5,63.5,54.4,42.5,35.0,34.2,27.1,24.9,23.7,23.7,23.7,14.0 \mathrm{ppm} ;[\alpha]_{\mathrm{D}}{ }^{20}=-32(c=$ 2.54 in $\mathrm{AcCN})$.

\section{Summary: \\ The crystal structure of 80 was characterized by single crystal X-ray diffraction. The X-ray data were collected using a colorless needle shaped crystal grown from $\mathrm{MeOH}-\mathrm{H}_{2} \mathrm{O}$. There is one molecule per asymmetric unit. The stereochemistry of the $\mathrm{S}$ atom was determined to be $(2 R, 3 S)$ and $R$ at sulfure based on the refinement of anomalous scattering.}

\section{Structure by X-ray crystallography:}






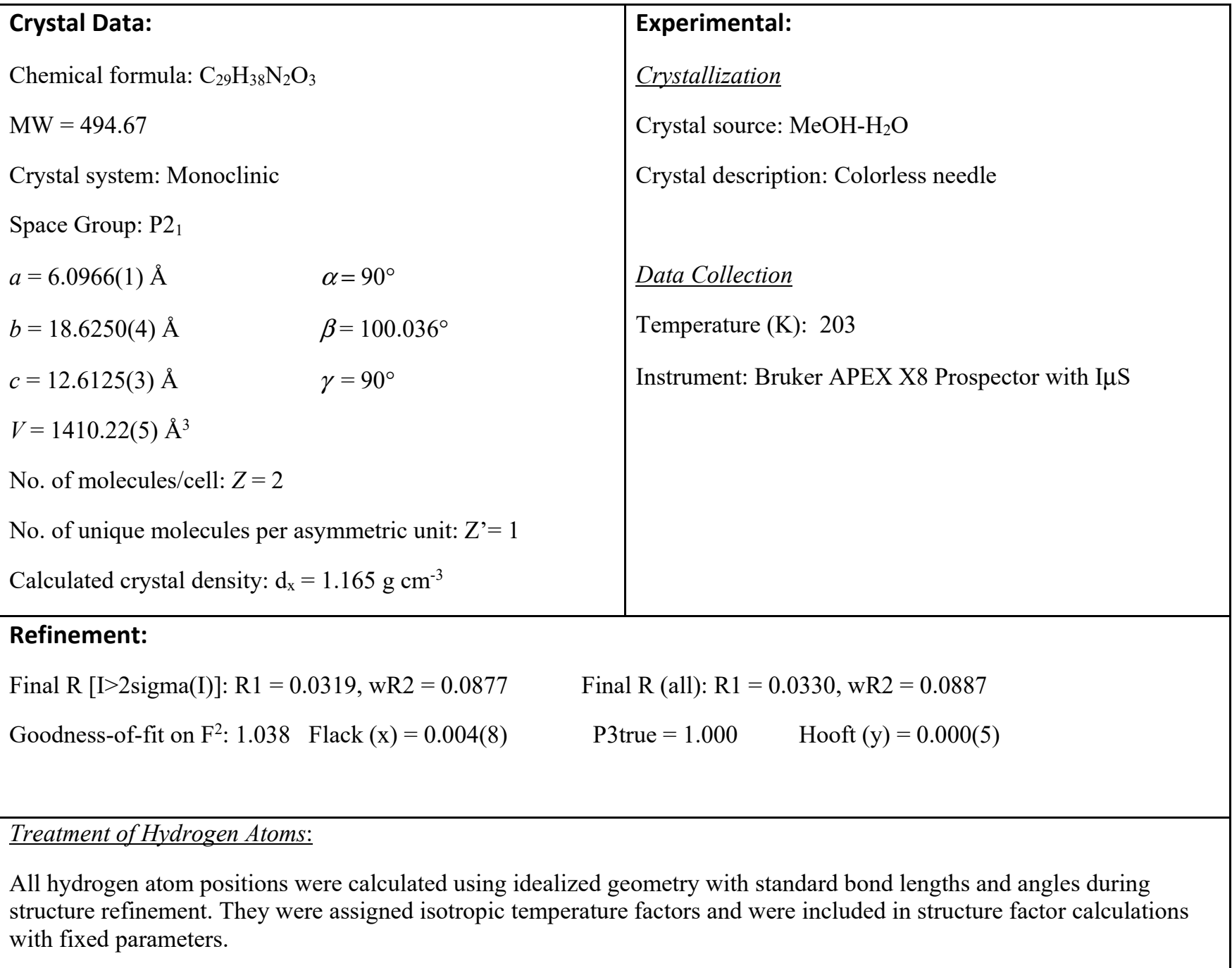

(R)-2-phenyl-1-((R)-(2,4,6-triisopropylphenyl)sulfinyl)pyrrolidine-
3,3-dicarbonitrile (80). Compound was synthesized according to
General Procedure B with the exception that after $2 \mathrm{~h}$ reaction time at -
$78^{\circ} \mathrm{C}$, the reaction was allowed to warm slowly overnight. The
product was purified using an ISCO teledyne instrument with a $12 \mathrm{~g}$
silica gel column while eluting with hexanes and EtOAc (gradient of 0
to $100 \%$ EtOAc) and was isolated as a white solid (86 $\mathrm{mg}, 67 \%$ yield). UPLC $t r=1.68 \mathrm{~min}$ (Instrument A); HRMS (ESI) $m / z$ calcd for $\mathrm{C} 27 \mathrm{H} 33 \mathrm{~N} 3 \mathrm{OS}[\mathrm{M}+\mathrm{H}]^{+}$ 448.2417, found 448.2423; ${ }^{1} \mathrm{H}$ NMR (CHLOROFORM-d, 400MHz) $\delta 7.58-7.63(\mathrm{~m}, 2 \mathrm{H}), 7.46-$ $7.55(\mathrm{~m}, 3 \mathrm{H}), 7.08(\mathrm{~s}, 2 \mathrm{H}), 4.89(\mathrm{~s}, 1 \mathrm{H}), 3.93-4.10(\mathrm{~m}, 1 \mathrm{H}), 3.87$ (br s, 2H), 3.36 (dt, $J=10.0,6.8$ $\mathrm{Hz}, 1 \mathrm{H}), 2.77-2.92(\mathrm{~m}, 3 \mathrm{H}), 1.15-1.29(\mathrm{~m}, 18 \mathrm{H}) ;{ }^{13} \mathrm{C}$ NMR (CHLOROFORM-d, 101MHz) $\delta$ 152.8, 149.8, 131.4, 131.0, 130.8, 129.0, 129.0, 123.3, 113.8, 113.5, 71.8, 42.0, 41.4, 36.4, 34.2, $27.2,24.9,23.7 \mathrm{ppm} ;[\alpha]_{\mathrm{D}}{ }^{20}=-59(c=1.76$ in $\mathrm{AcCN})$. 


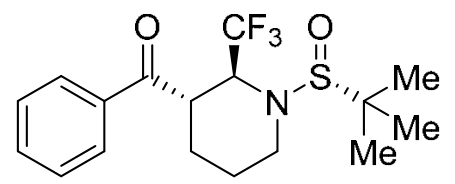

((2S,3S)-1-((R)-tert-butylsulfinyl)-2-(trifluoromethyl)piperidin-3yl)(phenyl)methanone (8p). Compound was synthesized according to General Procedure B. The product was purified using an ISCO teledyne instrument with a $12 \mathrm{~g}$ silica gel column while eluting with hexanes and EtOAc (gradient of 0 to 100\% EtOAc) and was isolated as a white solid (108 mg combined, 75\% yield). Data for major trans- isomer, UPLC $t r=1.30 \mathrm{~min}$ (Instrument A); HRMS (ESI) $\mathrm{m} / \mathrm{z}$ calcd for $\mathrm{C} 17 \mathrm{H} 22 \mathrm{NO} 2 \mathrm{~F} 3 \mathrm{~S}[\mathrm{M}+\mathrm{H}]^{+}$362.1396, found $362.1401 ;{ }^{1} \mathrm{H}$ NMR $(400 \mathrm{MHz}$, CHLOROFORM-d) $\delta 7.95(\mathrm{~d}, J=7.0 \mathrm{~Hz}, 2 \mathrm{H}), 7.64-7.57(\mathrm{~m}, 1 \mathrm{H}), 7.51(\mathrm{t}, J=7.6 \mathrm{~Hz}, 2 \mathrm{H}), 4.66$ $(\mathrm{qd}, J=9.1,3.5 \mathrm{~Hz}, 1 \mathrm{H}), 3.79-3.71(\mathrm{~m}, 2 \mathrm{H}), 3.07-2.99(\mathrm{~m}, 1 \mathrm{H}), 2.14-2.05(\mathrm{~m}, 1 \mathrm{H}), 1.96-1.77$ $(\mathrm{m}, 1 \mathrm{H}), 1.76-1.61(\mathrm{~m}, 2 \mathrm{H}), 1.53-1.36(\mathrm{~m}, 1 \mathrm{H}), 1.30-1.23(\mathrm{~m}, 9 \mathrm{H}) ;{ }^{13} \mathrm{C}$ NMR $(101 \mathrm{MHz}$ CHLOROFORM-d) $\delta 199.3,135.1,133.4,128.9$ (2C), 128.5 (2C), 126.4 (q, J=284 Hz, 1C), 60.9 (q, $J=29 \mathrm{~Hz}, 1 \mathrm{C}), 59.6,40.3,37.7,23.7$ (3C), 23.3, 21.5.

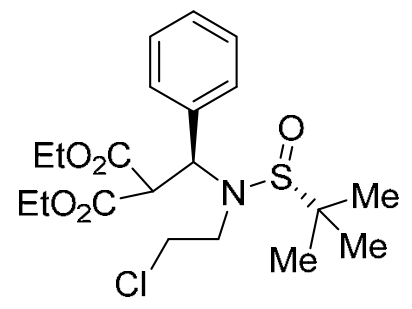

Diethyl 2-((S)-(((R)-tert-butylsulfinyl)(2chloroethyl)amino)(phenyl)methyl)malonate (9a). Compound was synthesized according to General Procedure B. The product was purified using an ISCO teledyne instrument with a $12 \mathrm{~g}$ silica gel column while eluting with hexanes and EtOAc (gradient of 0 to $100 \%$ EtOAc) and was isolated as a diastereomeric mixture of $\mathbf{9 a}$ and $\mathbf{9 b}$ ( $161 \mathrm{mg}, 68 \%$ yield). The diastermeric mixture was separated via preparative LC/MS with the following conditions: Column: XBridge C18, $200 \mathrm{~mm} \times 19 \mathrm{~mm}, 5-$ $\mu \mathrm{m}$ particles; Mobile Phase A: 5:95 acetonitrile: water with 0.1\% trifluoroacetic acid; Mobile Phase B: 95:5 acetonitrile: water with 0.1\% trifluoroacetic acid; Gradient: a 0-minute hold at $40 \%$ B, $40-80 \%$ B over 25 minutes, then a 4-minute hold at $100 \%$ B; Flow Rate: $20 \mathrm{~mL} / \mathrm{min}$; Column Temperature: $25 \mathrm{C}$. Fraction collection was triggered by MS and UV signals. Fractions containing the desired product were combined and dried via centrifugal evaporation. The material was further purified via preparative LC/MS with the following conditions: Column: XBridge C18, $200 \mathrm{~mm}$ x $19 \mathrm{~mm}, 5-\mu \mathrm{m}$ particles; Mobile Phase A: 5:95 acetonitrile: water with 10-mM ammonium acetate; Mobile Phase B: 95:5 acetonitrile: water with 10-mM ammonium acetate; Gradient: a 0-minute hold at 30\% B, 30-70\% B over 20 minutes, then a 4-minute hold at $100 \%$ B; Flow Rate: $20 \mathrm{~mL} / \mathrm{min}$; Column Temperature: $25 \mathrm{C}$. Fraction collection was triggered by MS signals. Fractions containing the desired product were combined and dried via centrifugal evaporation. The material was further purified via preparative LC/MS with the following conditions: Column: XBridge C18, $200 \mathrm{~mm}$ x $19 \mathrm{~mm}$, 5- $\mu \mathrm{m}$ particles; Mobile Phase A: 5:95 acetonitrile: water with 10-mM ammonium acetate; Mobile Phase B: 95:5 acetonitrile: water with 10-mM ammonium acetate; Gradient: a 0-minute hold at 35\% B, 35-75\% B over 25 minutes, then a 4-minute hold at $100 \%$ B; Flow Rate: $20 \mathrm{~mL} / \mathrm{min}$; Column Temperature: $25 \mathrm{C}$. Fraction collection was triggered by MS signals. Fractions containing the desired product were combined and dried via centrifugal evaporation. Data for major isomer 9a, UPLC tr $=1.33 \mathrm{~min}$ (Instrument A); HRMS (ESI) $\mathrm{m} / \mathrm{z}$ calcd for $\mathrm{C} 20 \mathrm{H} 30 \mathrm{ClNO} 5 \mathrm{~S}[\mathrm{M}+\mathrm{H}]^{+} 432.1606$, found 432.1616; ${ }^{1} \mathrm{H}$ NMR (CHLOROFORM-d, 400MHz) $\delta$ 7.44-7.49 (m, 2H), 7.32-7.39 (m, 3H), 4.72 (d, $J=11.8$ $\mathrm{Hz}, 1 \mathrm{H}), 4.38(\mathrm{~d}, J=11.9 \mathrm{~Hz}, 1 \mathrm{H}), 4.22(\mathrm{qd}, J=7.1,2.4 \mathrm{~Hz}, 2 \mathrm{H}), 3.79-4.01(\mathrm{~m}, 3 \mathrm{H}), 3.47-3.63$ (m, $2 \mathrm{H}), 2.76-2.86(\mathrm{~m}, 1 \mathrm{H}), 1.30(\mathrm{t}, J=7.2 \mathrm{~Hz}, 3 \mathrm{H}), 1.19$ (s, 9H), $0.86(\mathrm{t}, J=7.1 \mathrm{~Hz}, 3 \mathrm{H}) ;{ }^{13} \mathrm{C}$ NMR (CHLOROFORM-d, 101MHz) $\delta$ 166.9, 165.9, 135.0, 129.1, 129.0 (2C), 128.7 (2C), 65.0, 62.2, $61.8,58.2,56.7,44.8,40.6,23.6(3 \mathrm{C}), 14.0,13.5 \mathrm{ppm} ;[\alpha]_{\mathrm{D}}^{20}=-11.5$ ( $c=2.50$ in DMSO). 


\section{Reaction on a $1 \mathrm{~g}$ scale}<smiles>CC(C)(C)[S@](=O)N1CC[C@]2(S(=O)(=O)c3ccc(F)cc3)c3ccc(I)cc3CC[C@H]12</smiles>

(3aR,9bR)-3-((R)-tert-butylsulfinyl)-9b-((4fluorophenyl)sulfonyl)-7-iodo-2,3,3a,4,5,9b-hexahydro- $1 \mathrm{H}-$ benzo[e]indole (3e). To a solution of 4-((4-fluorophenyl)sulfonyl)7-iodo-1,2-dihydronaphthalene (1 g, $2.41 \mathrm{mmol})$ and $(\mathrm{R})-\mathrm{N}-(2-$ chloroethyl)-2-methylpropane-2-sulfinamide $(0.887 \mathrm{~g}, 4.83 \mathrm{mmol})$ in THF $(35 \mathrm{~mL})$ cooled in a dry ice-acetone bath was added a $1 \mathrm{M}$ solution of KOtBu in THF $(4.8 \mathrm{~mL})$ dropwise over $30 \mathrm{~min}$. The reaction mixture was stirred in the dry ice-acetone bath for an additional $30 \mathrm{~min}$. The reaction was completed. The reaction mixture was quenched with satd. $\mathrm{NH} 4 \mathrm{Cl}$, then extracted with ethyl acetate $(20 \mathrm{mLx} 3)$. The combined organic phases was washed with water, then brine, then concentrated. The product was purified using an ISCO teledyne instrument with a $80 \mathrm{~g}$ silica gel column while eluting with hexanes and EtOAc (gradient of 0 to $100 \%$ EtOAc) and was isolated as a white solid ( $1.11 \mathrm{~g}, 82 \%$ yield).

\section{Recrystallization}

The above material containing $2.8 \%$ of the minor diastereomer was recrystalized from $10 \mathrm{~mL}$ of ethyl acetate to give $833 \mathrm{mg}$ white crystal as first crop which contains $<0.1 \%$ of the minor diastereomer. The amount of minor diastereomer was determined by analytical SFC (Column: Chiralpak ID $\left(0.46^{\prime} 25 \mathrm{~cm}, 5 \mu \mathrm{m}\right)$, BPR pressure: 140 bars, Temperature: $40^{\circ} \mathrm{C}$, Flow rate: 3 $\mathrm{mL} / \mathrm{min}$, Mobile Phase: $\mathrm{CO} 2 / \mathrm{MeOH}$ (60/40), Detector Wavelength: $220 \mathrm{~nm}$ )

Analytical SFC:

$1 \mathrm{~g}$ scale reaction after silica gel purification



After recrystallization 


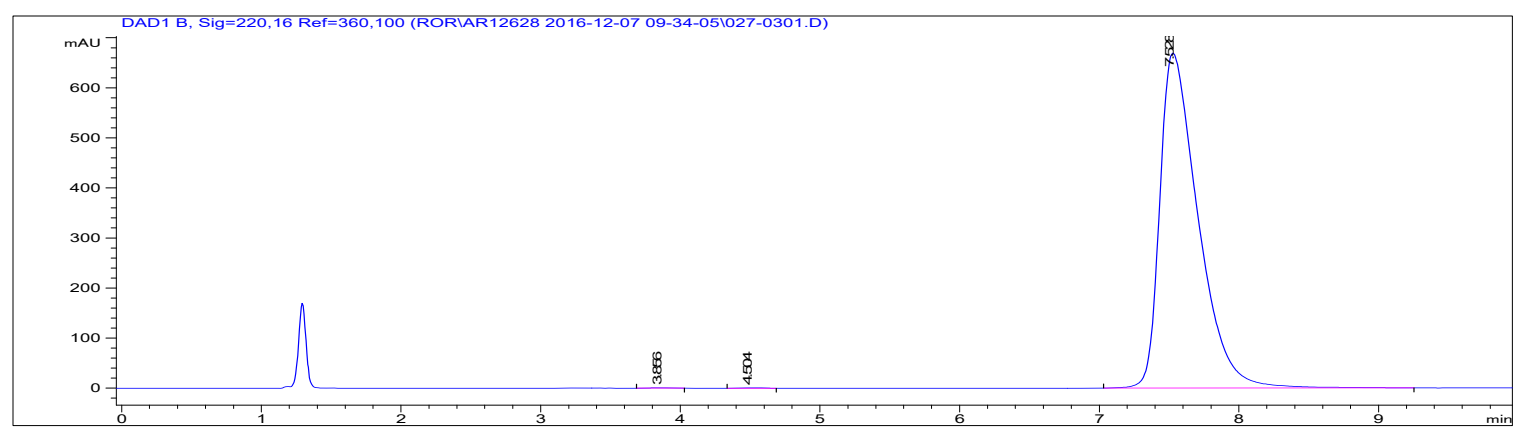

\section{Characterization data}

\section{${ }^{1} \mathrm{H}$ and ${ }^{13} \mathrm{C}$ Spectra}

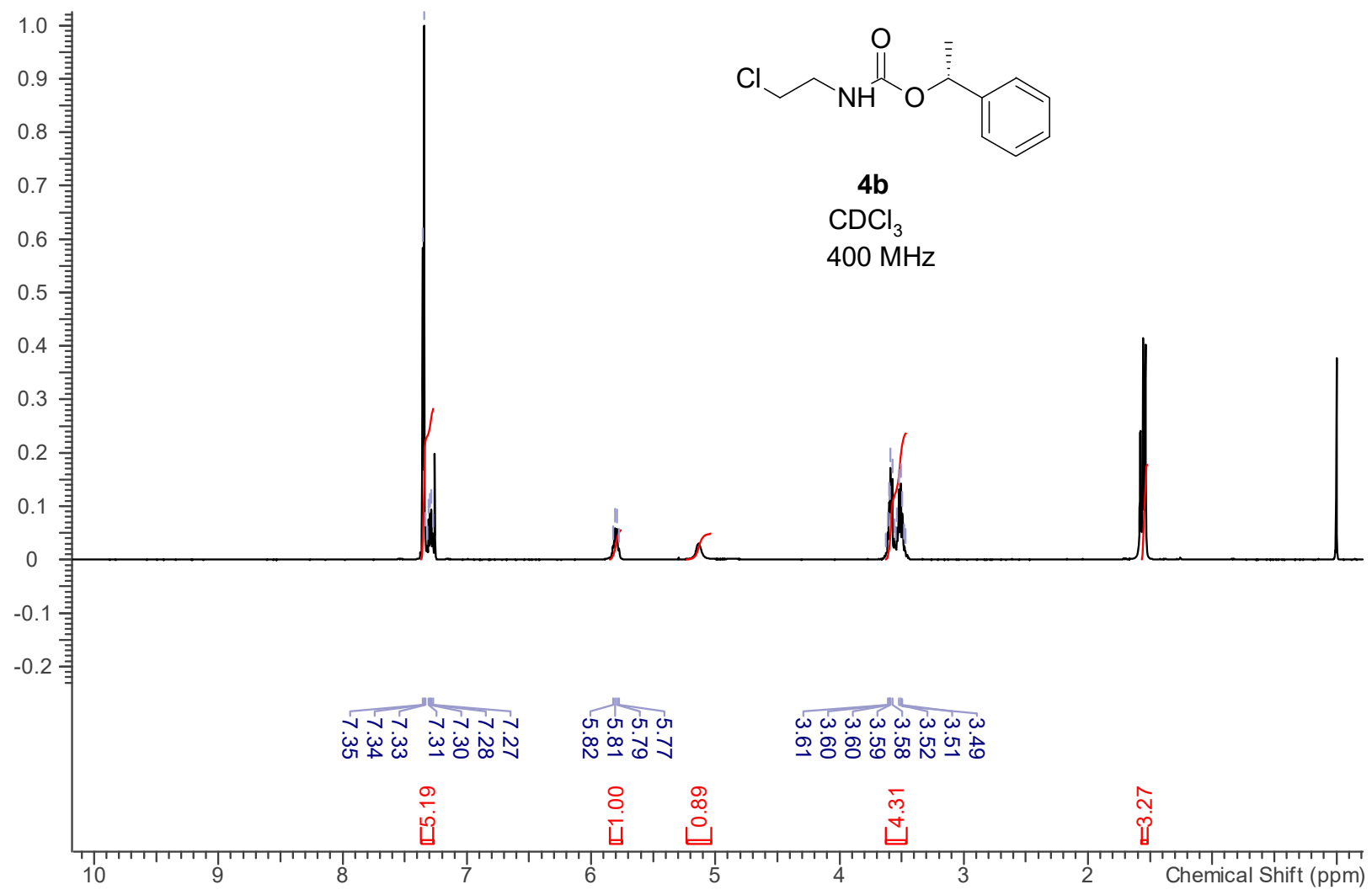



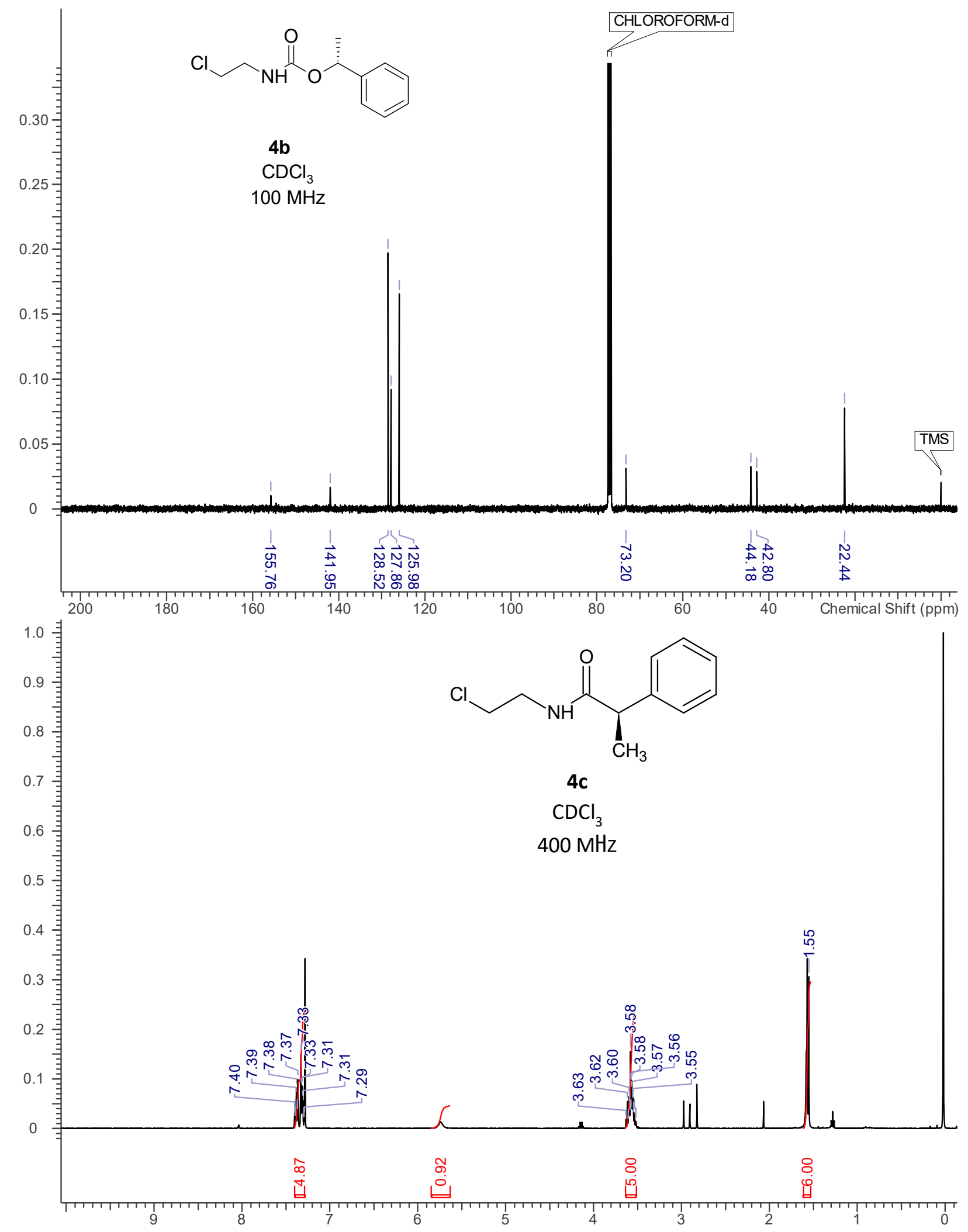


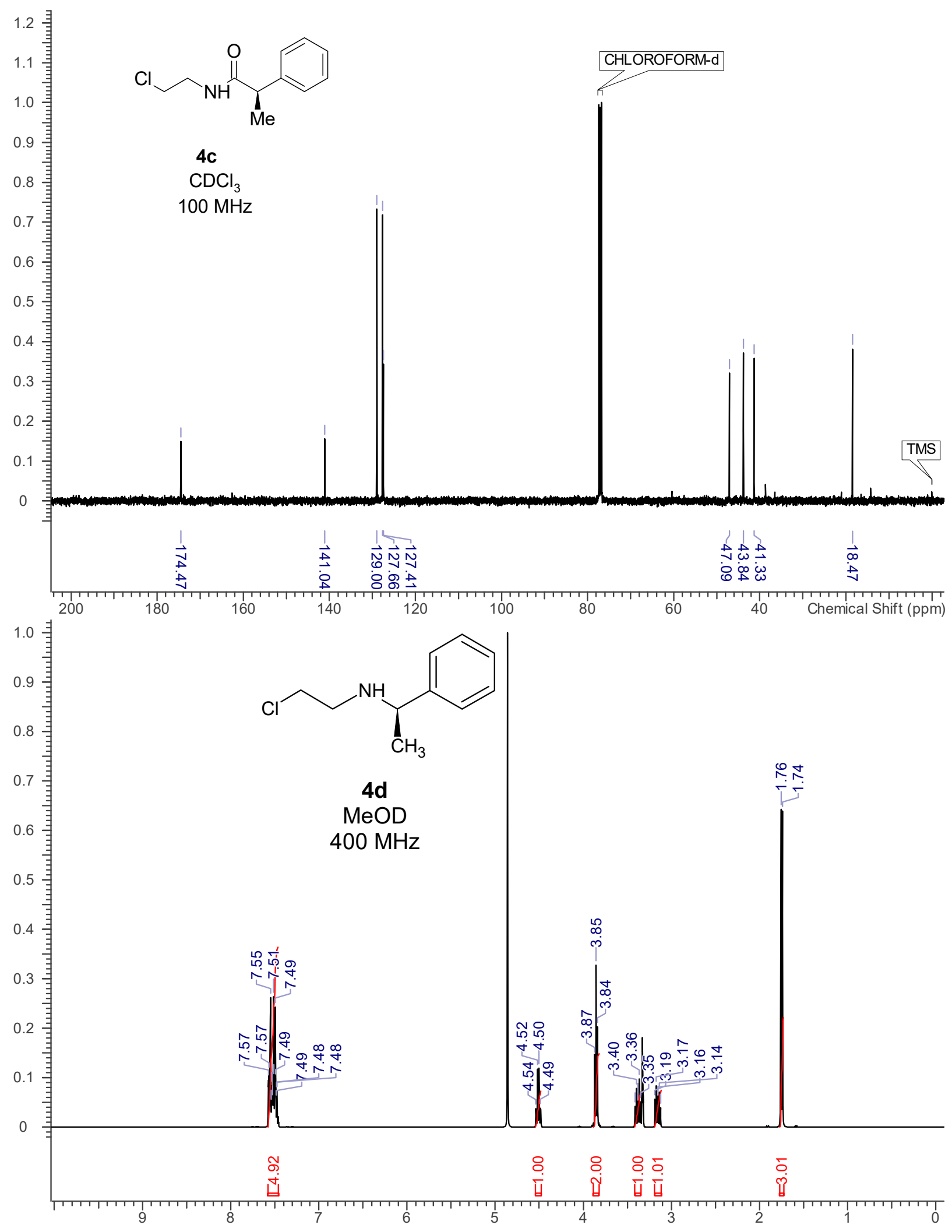



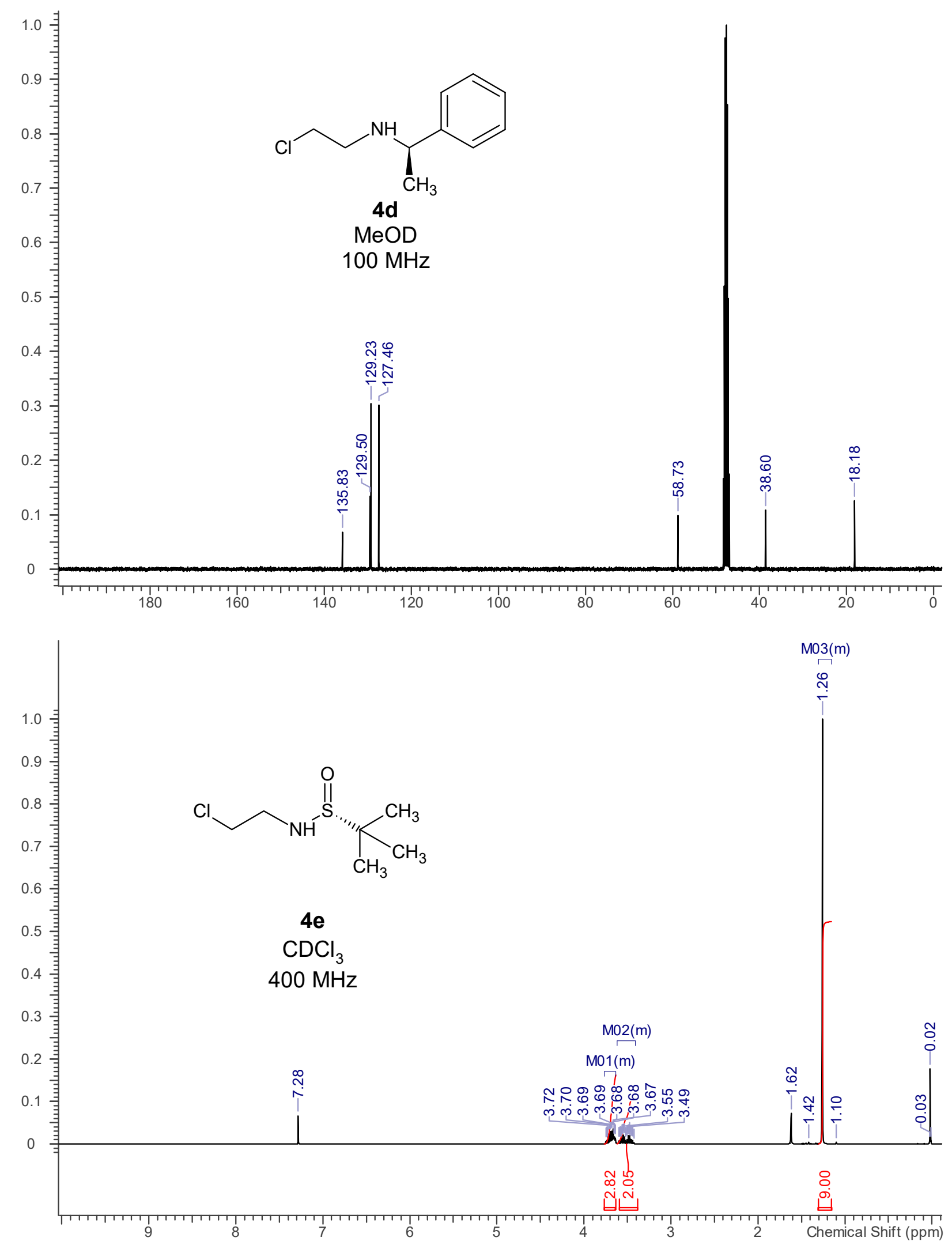

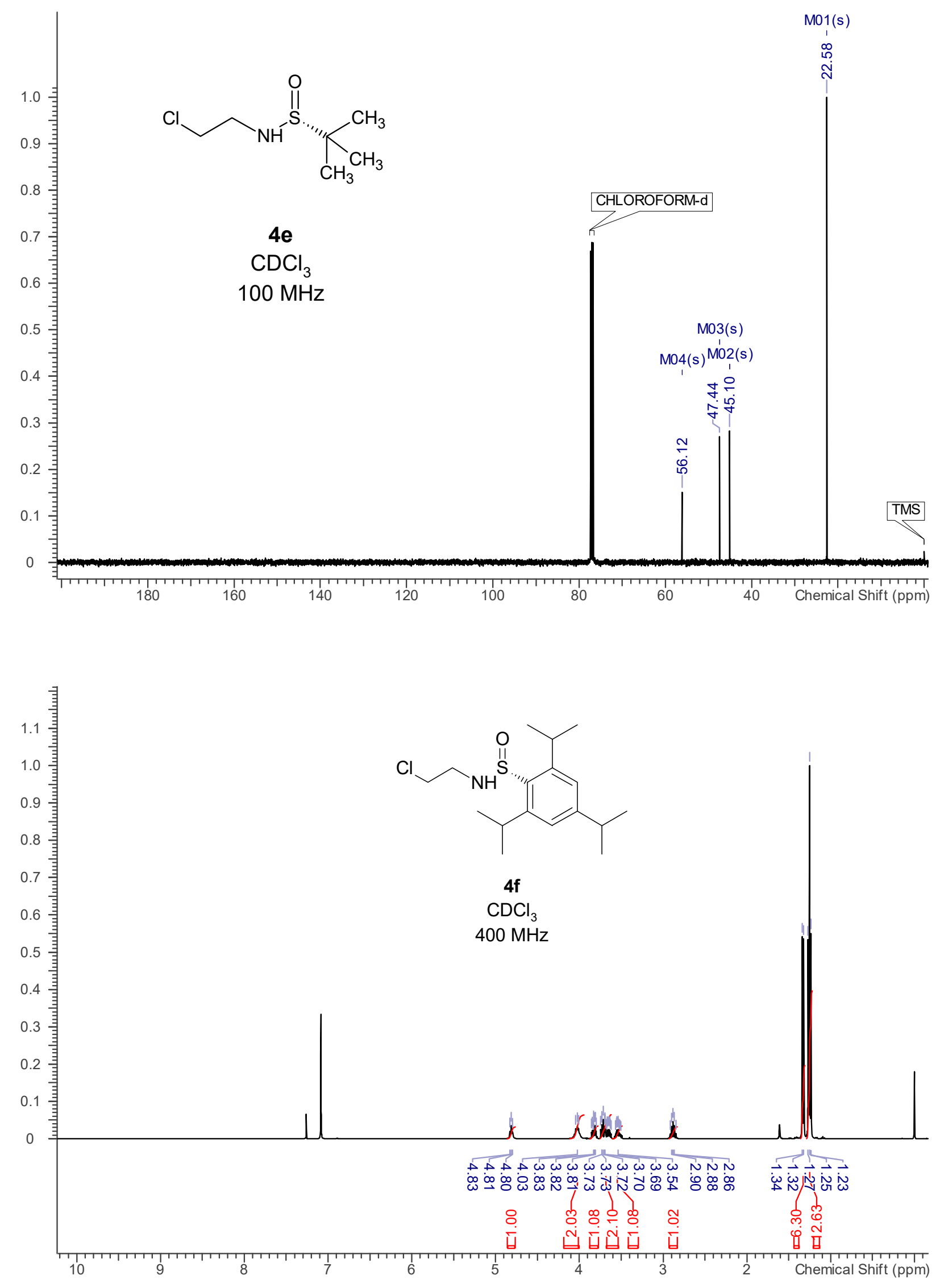


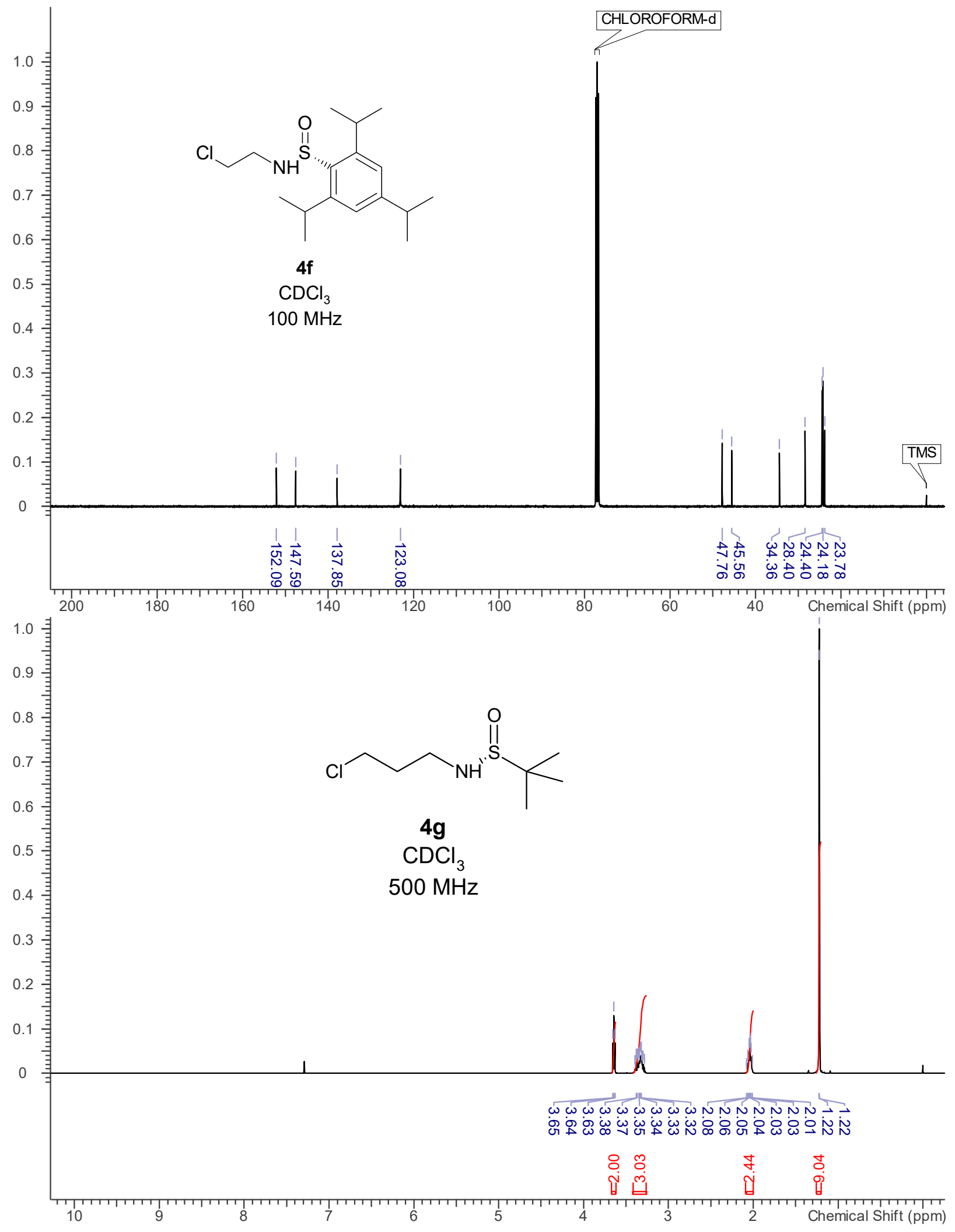




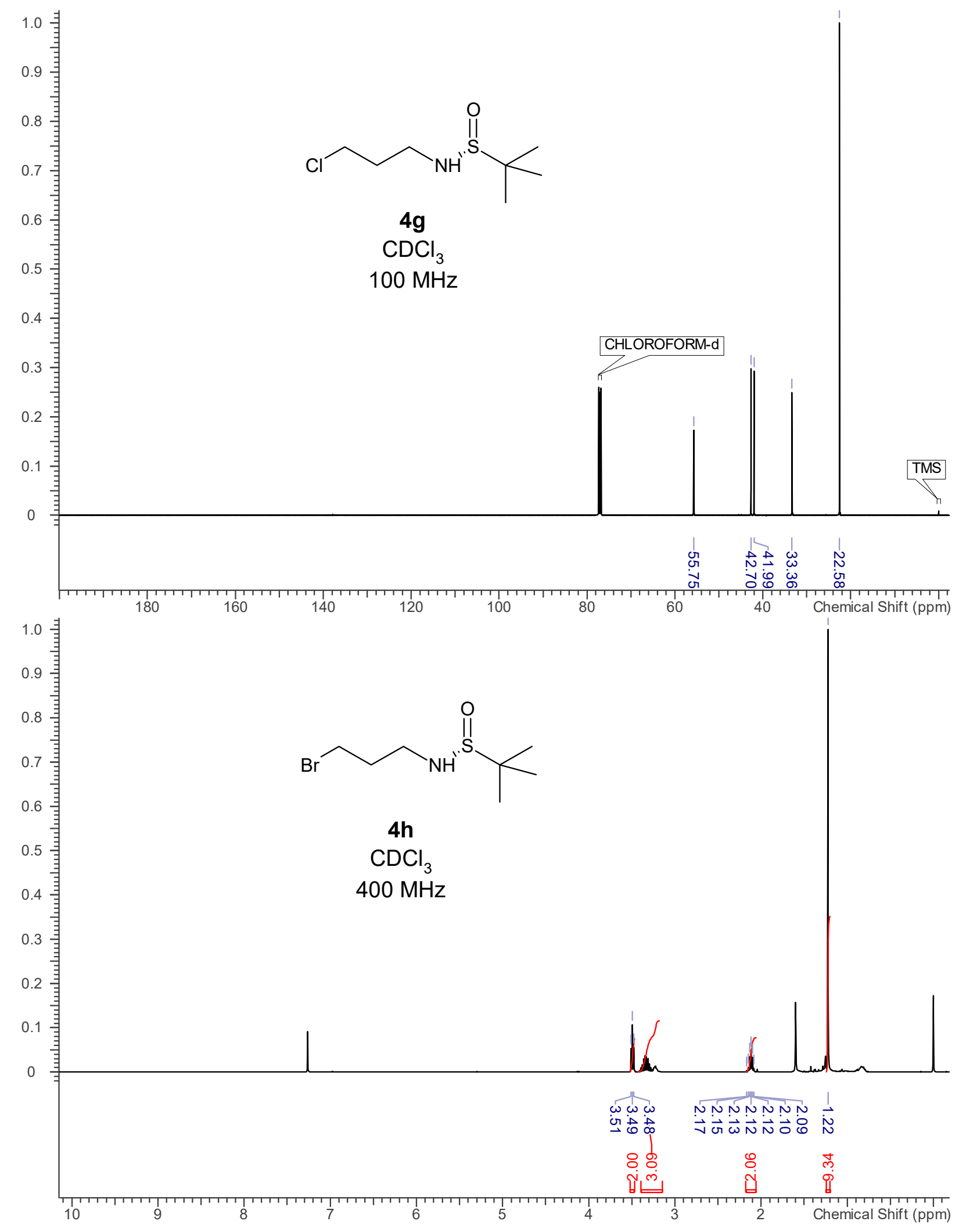









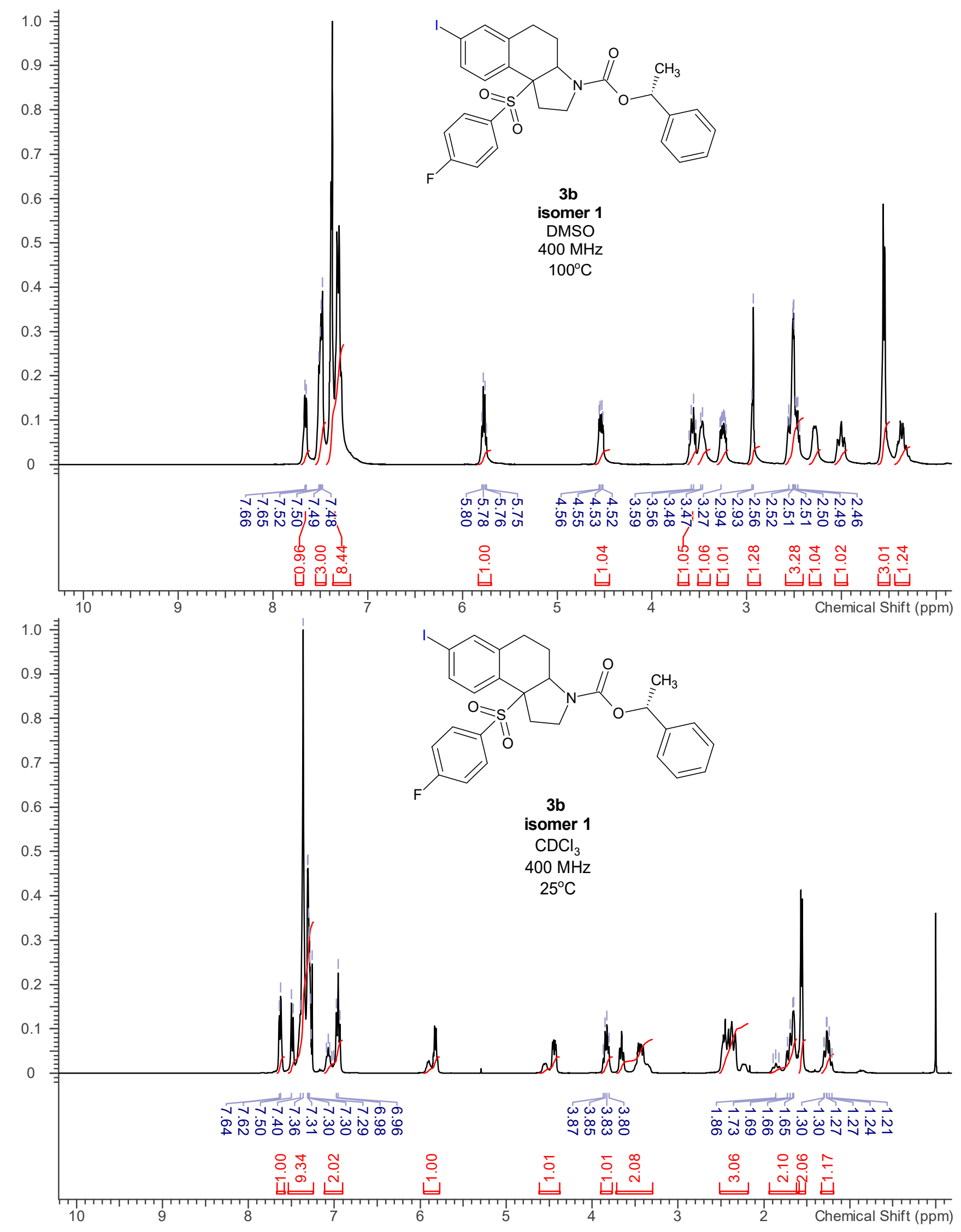



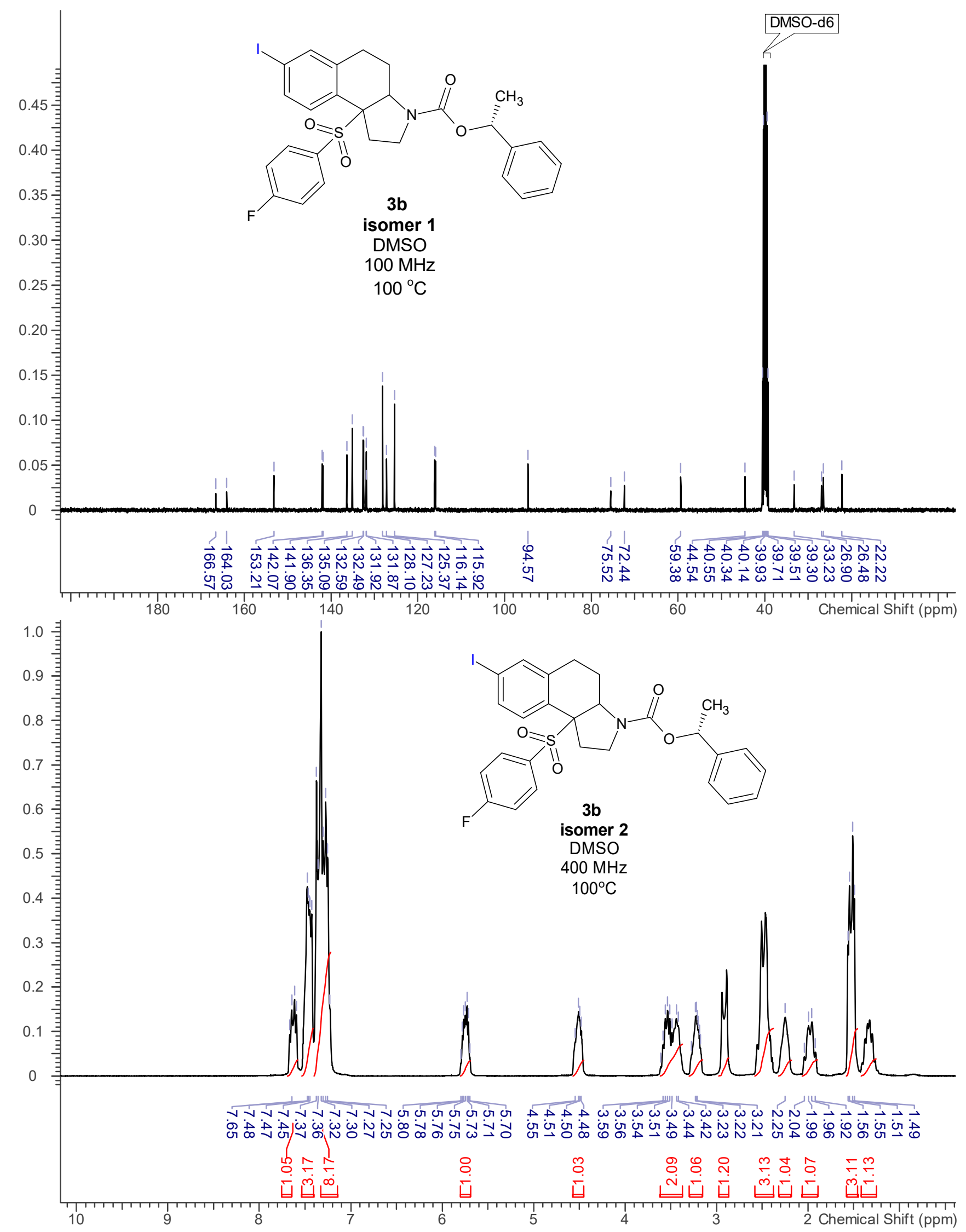


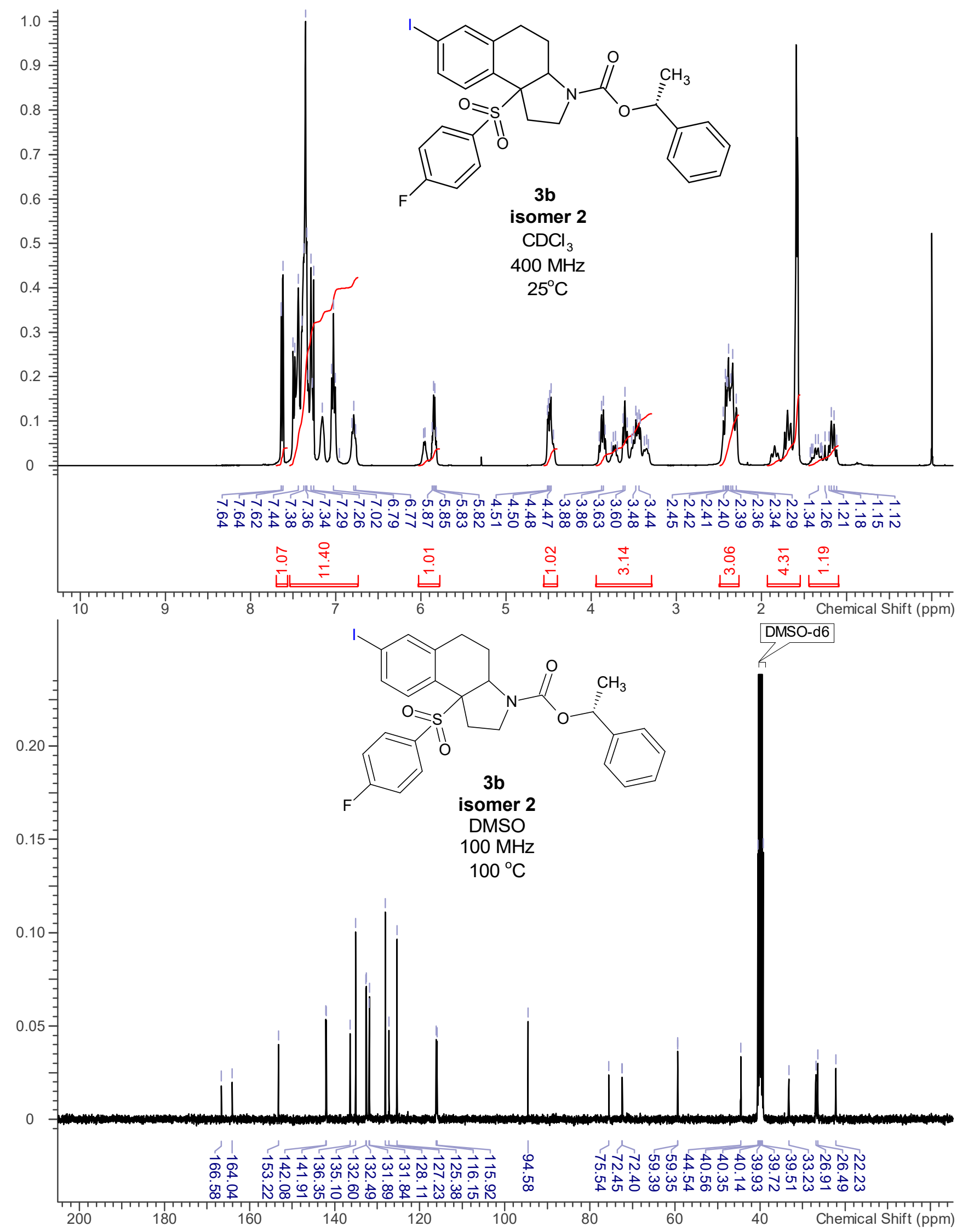



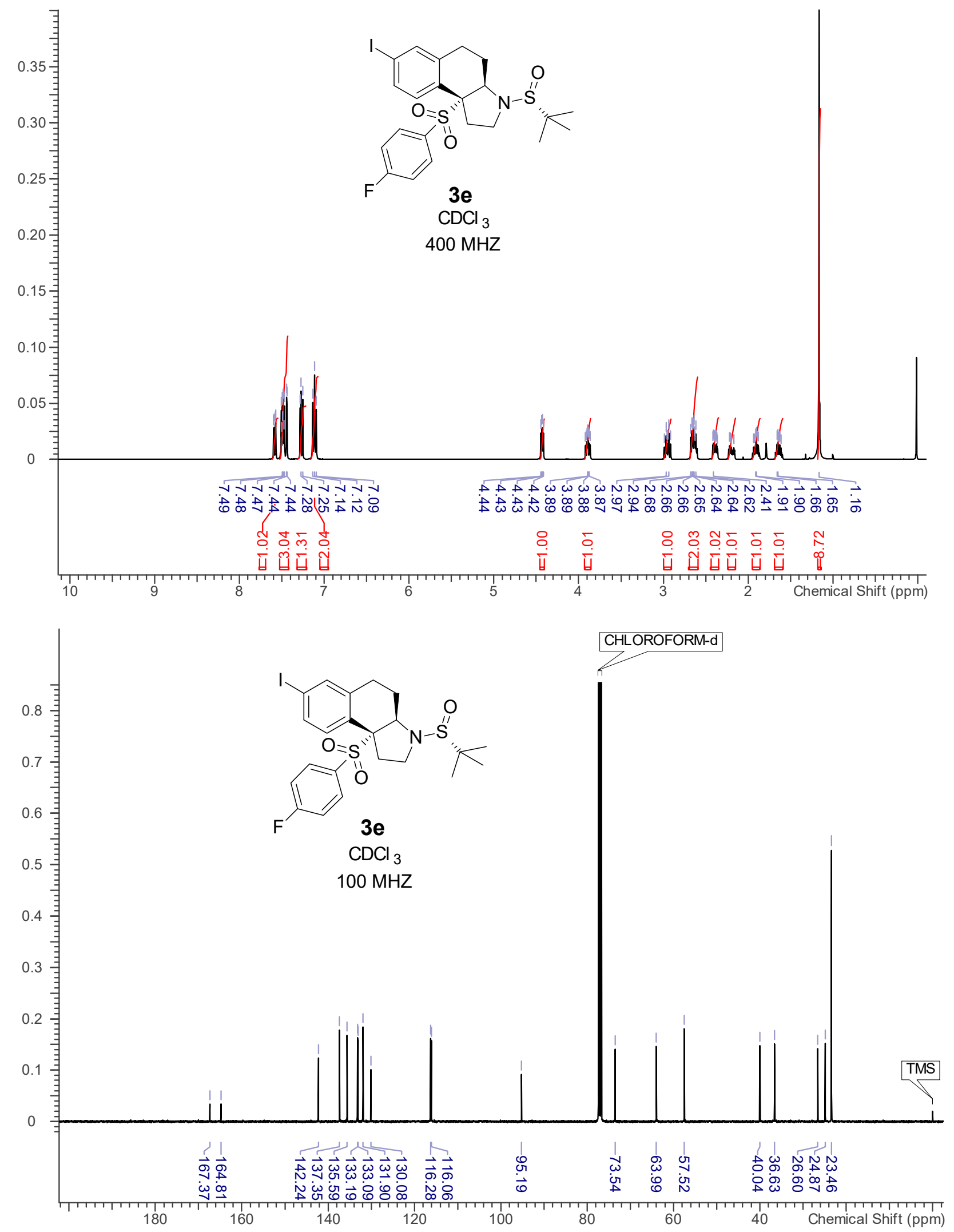


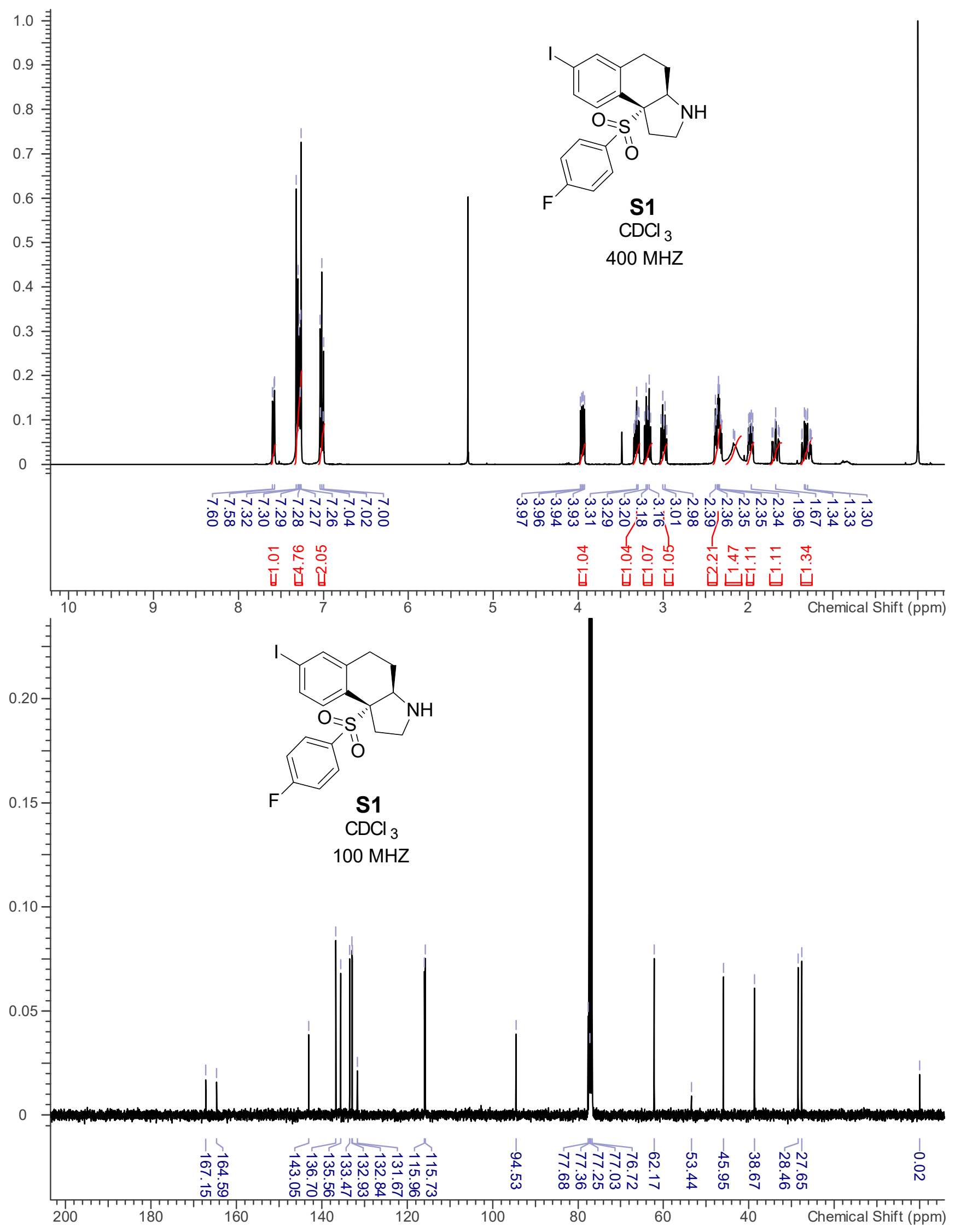




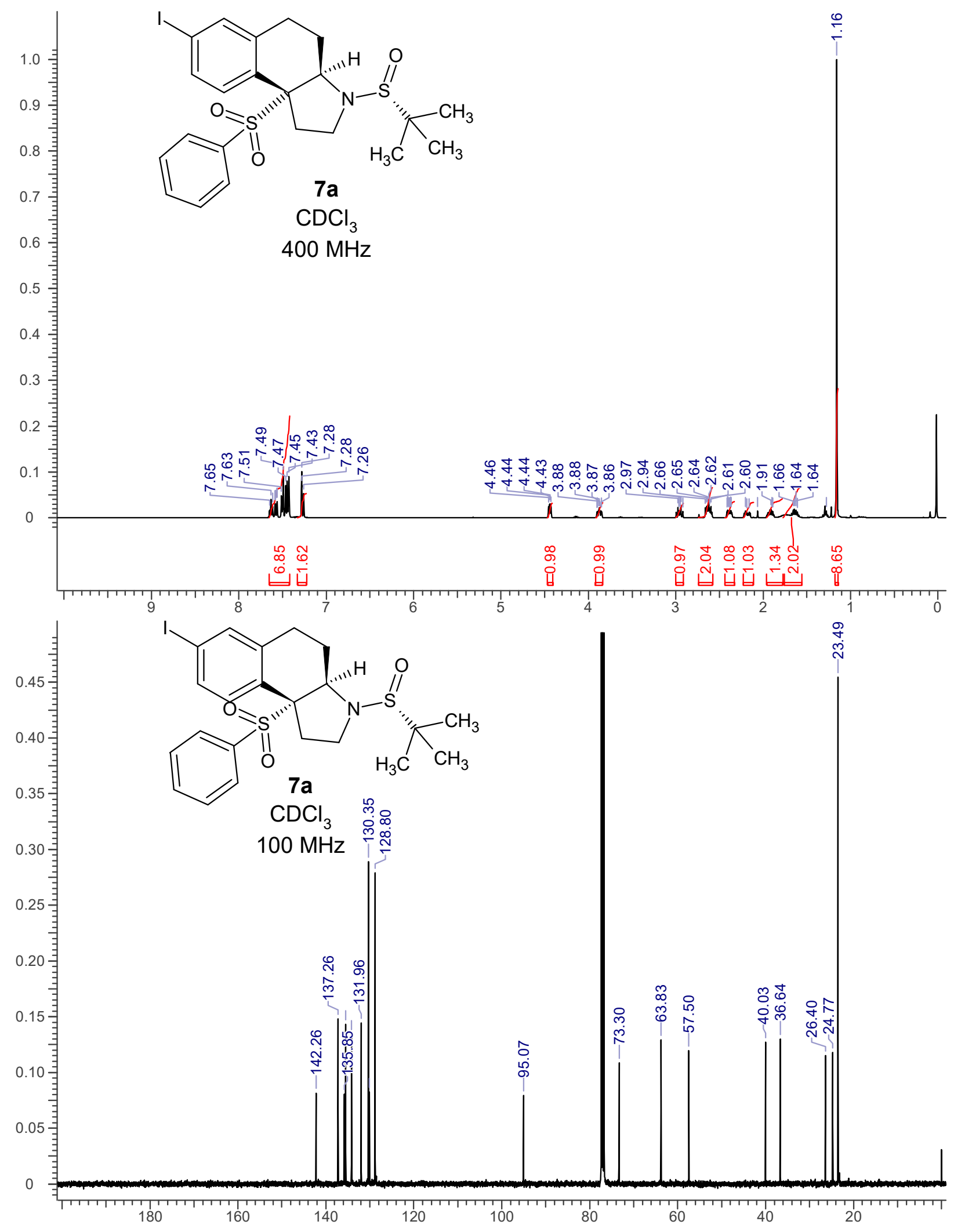



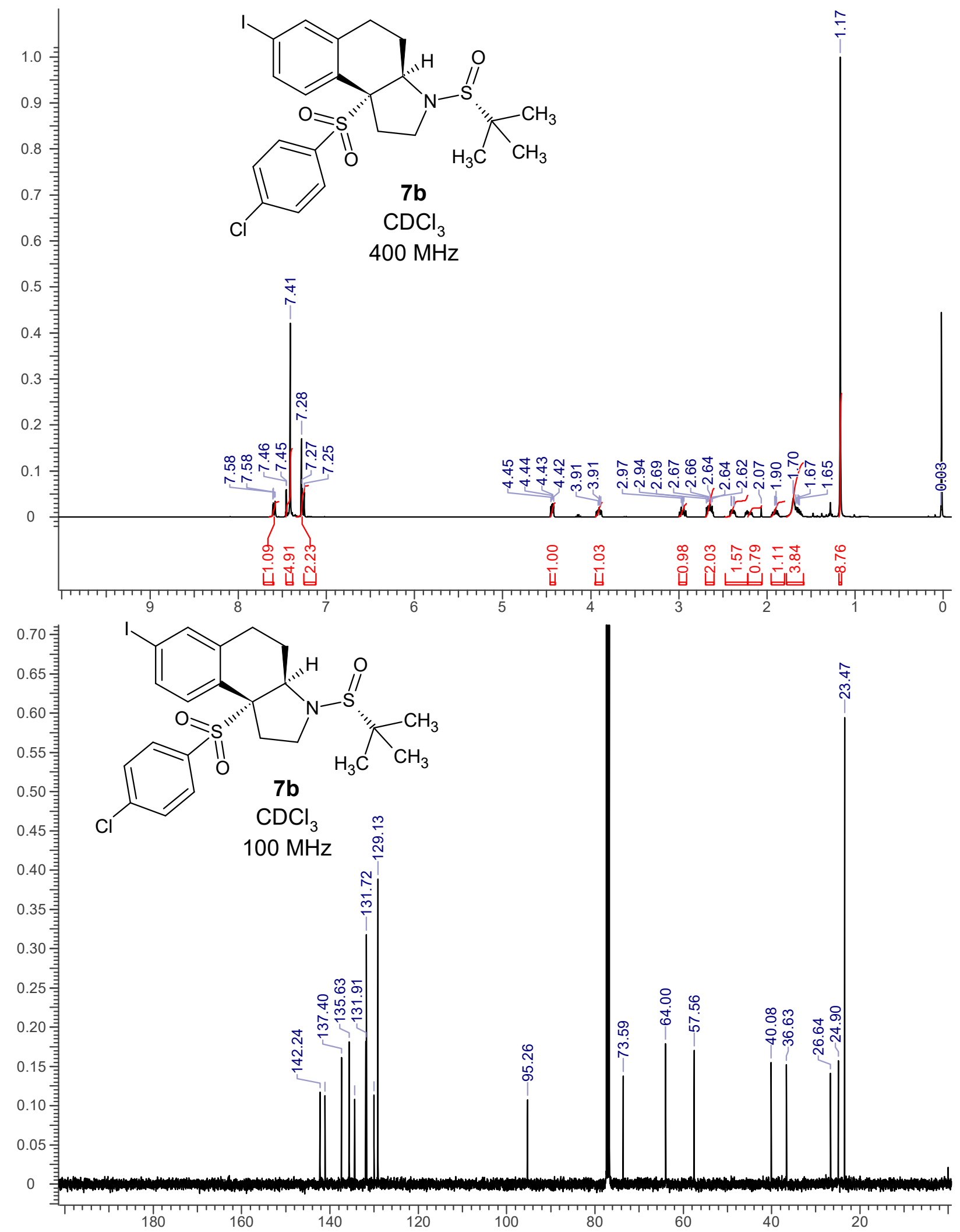


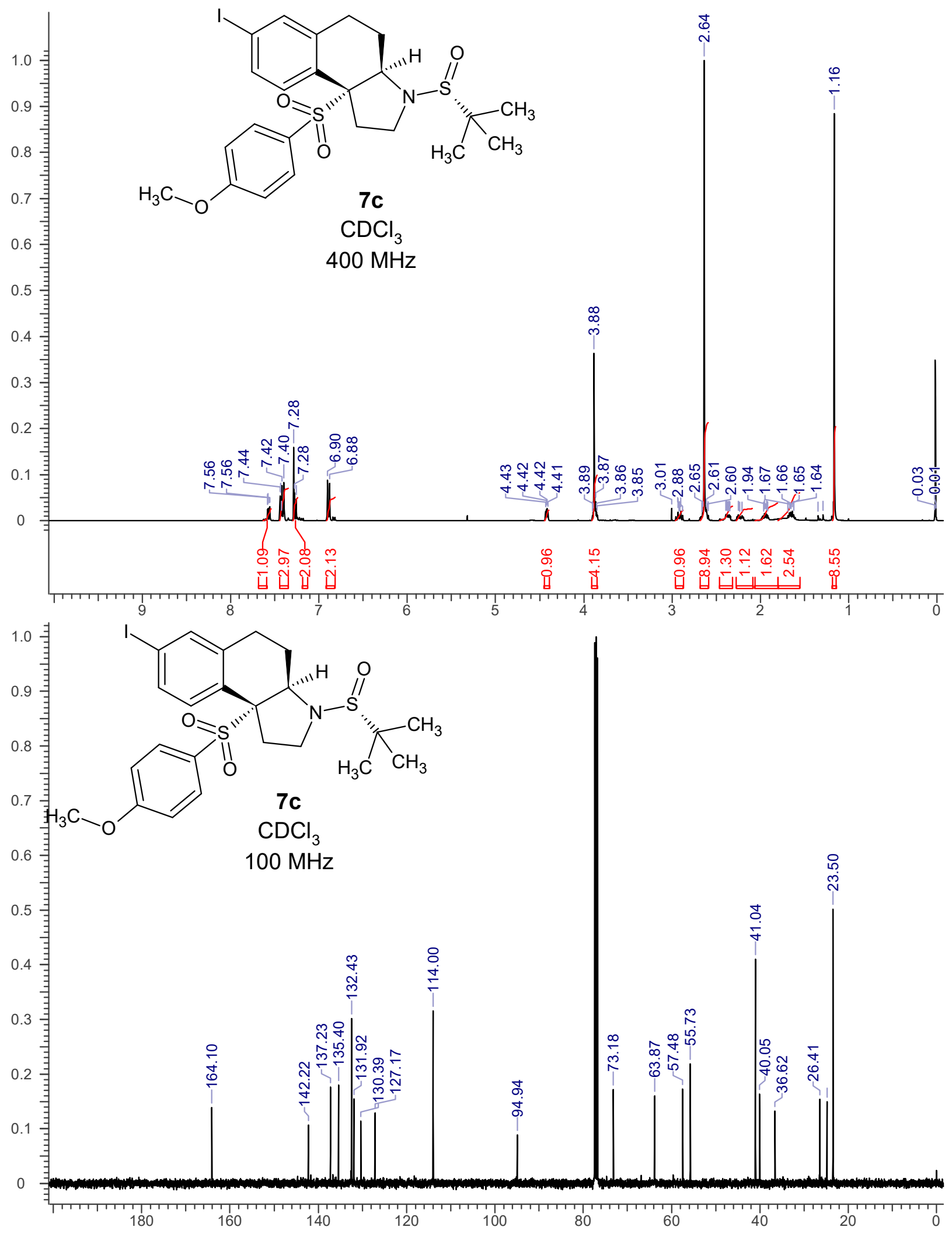




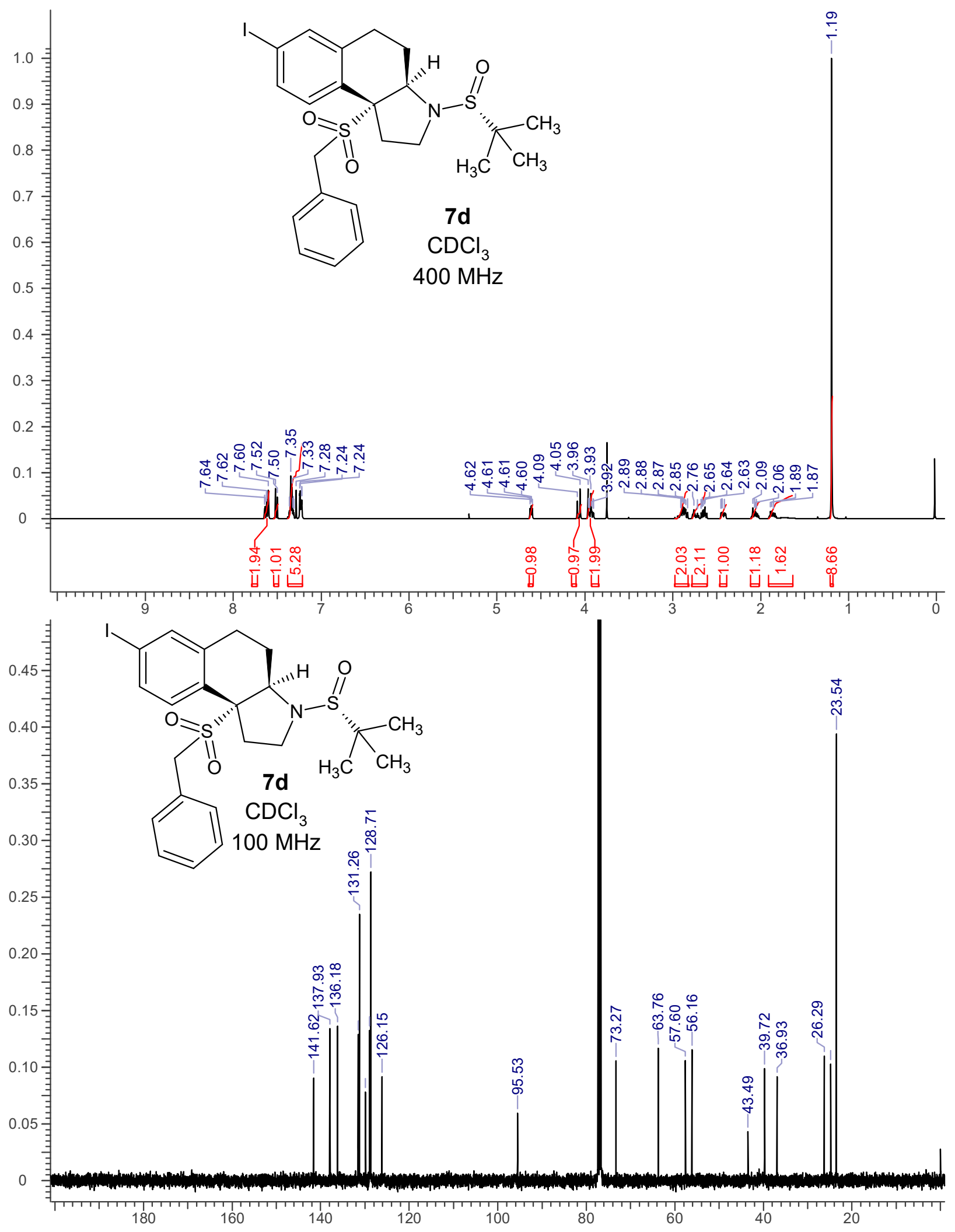






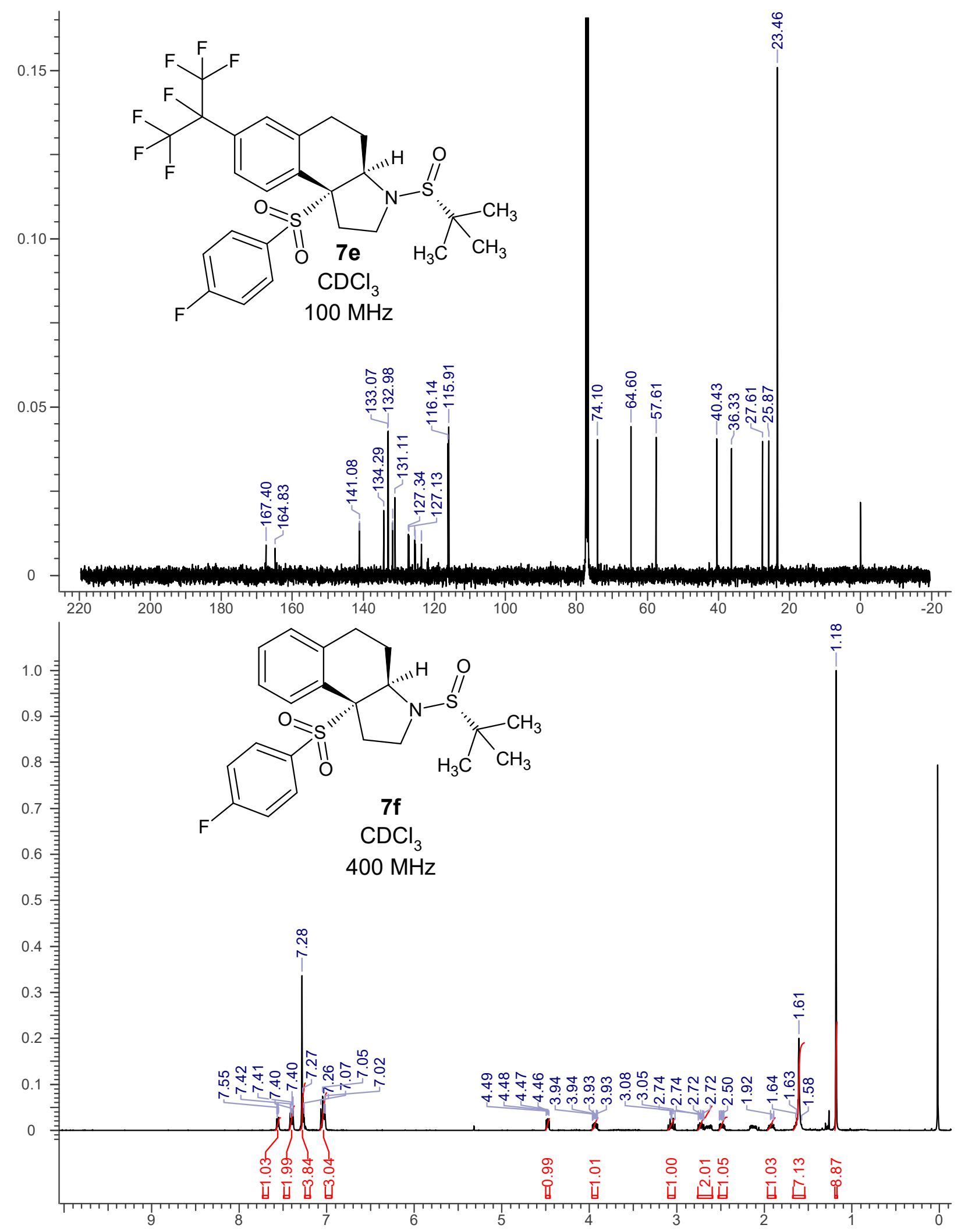




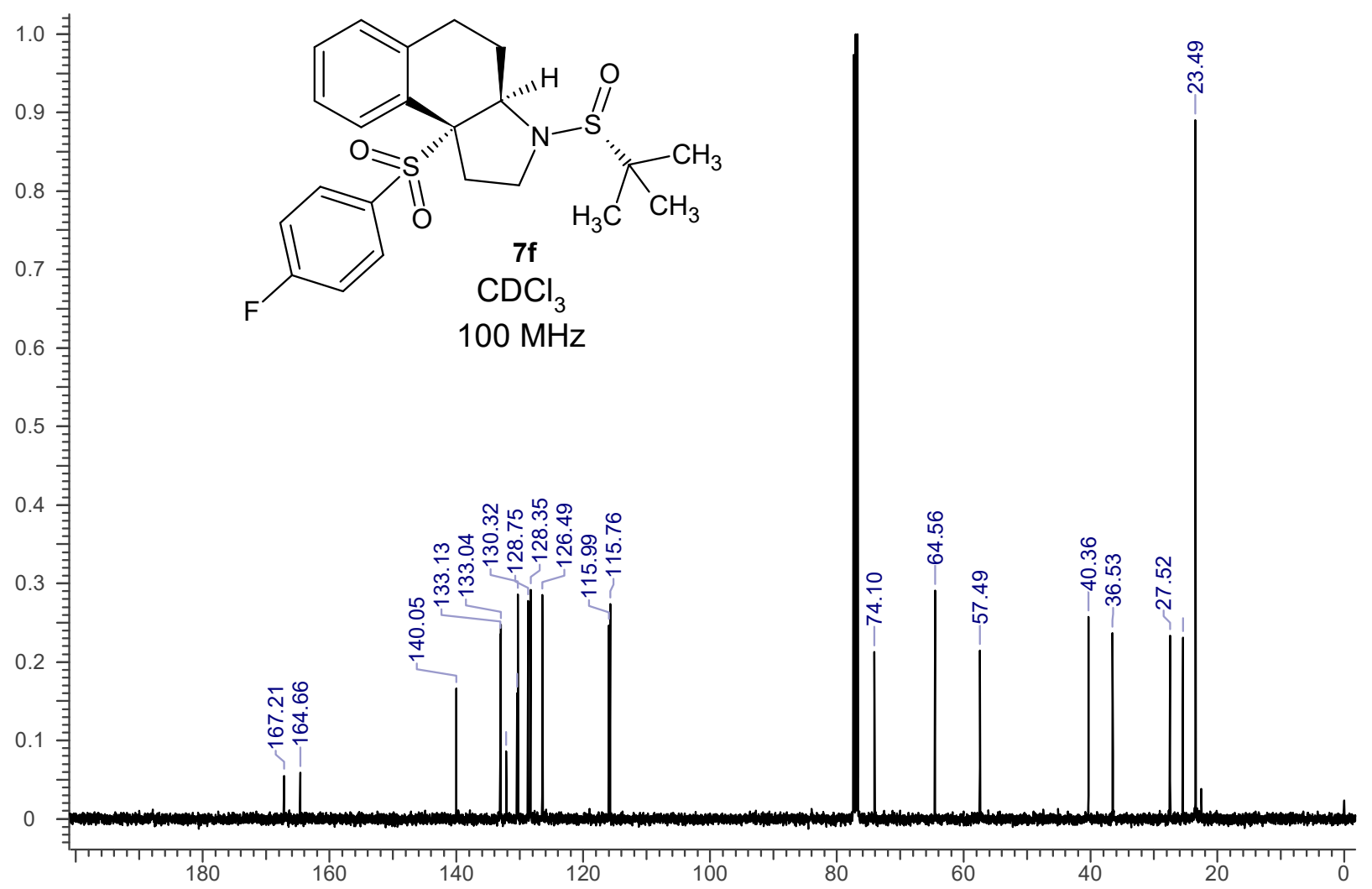




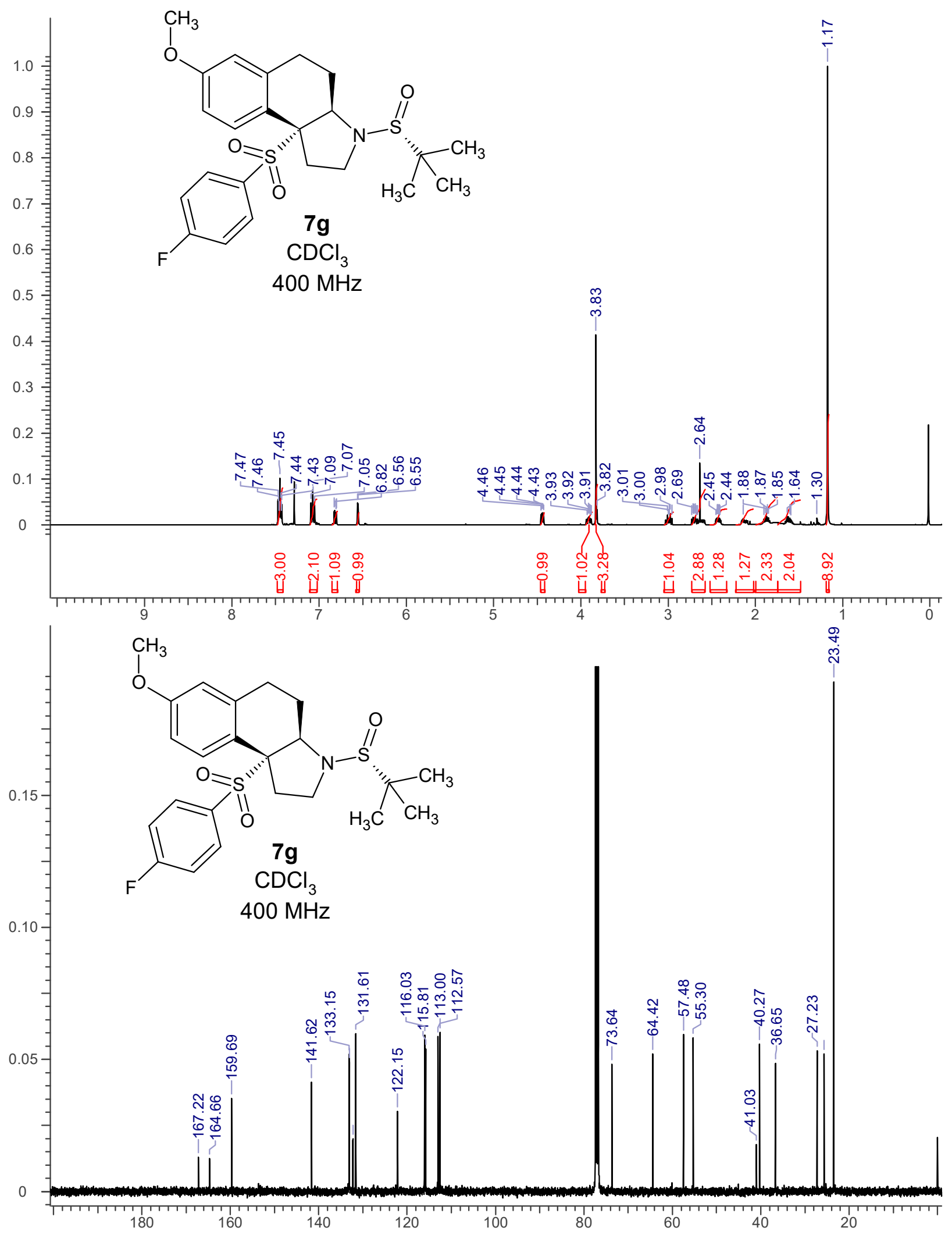




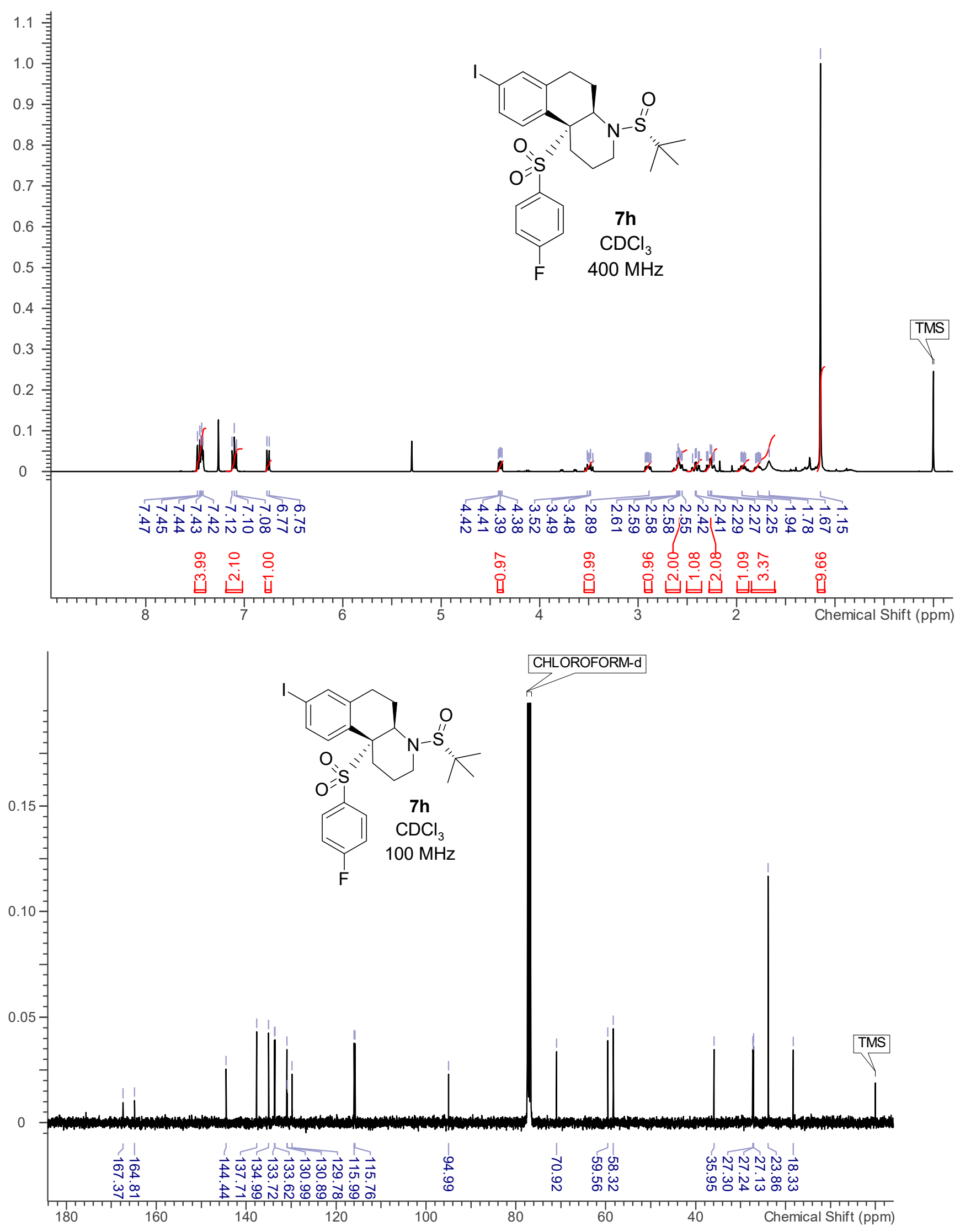




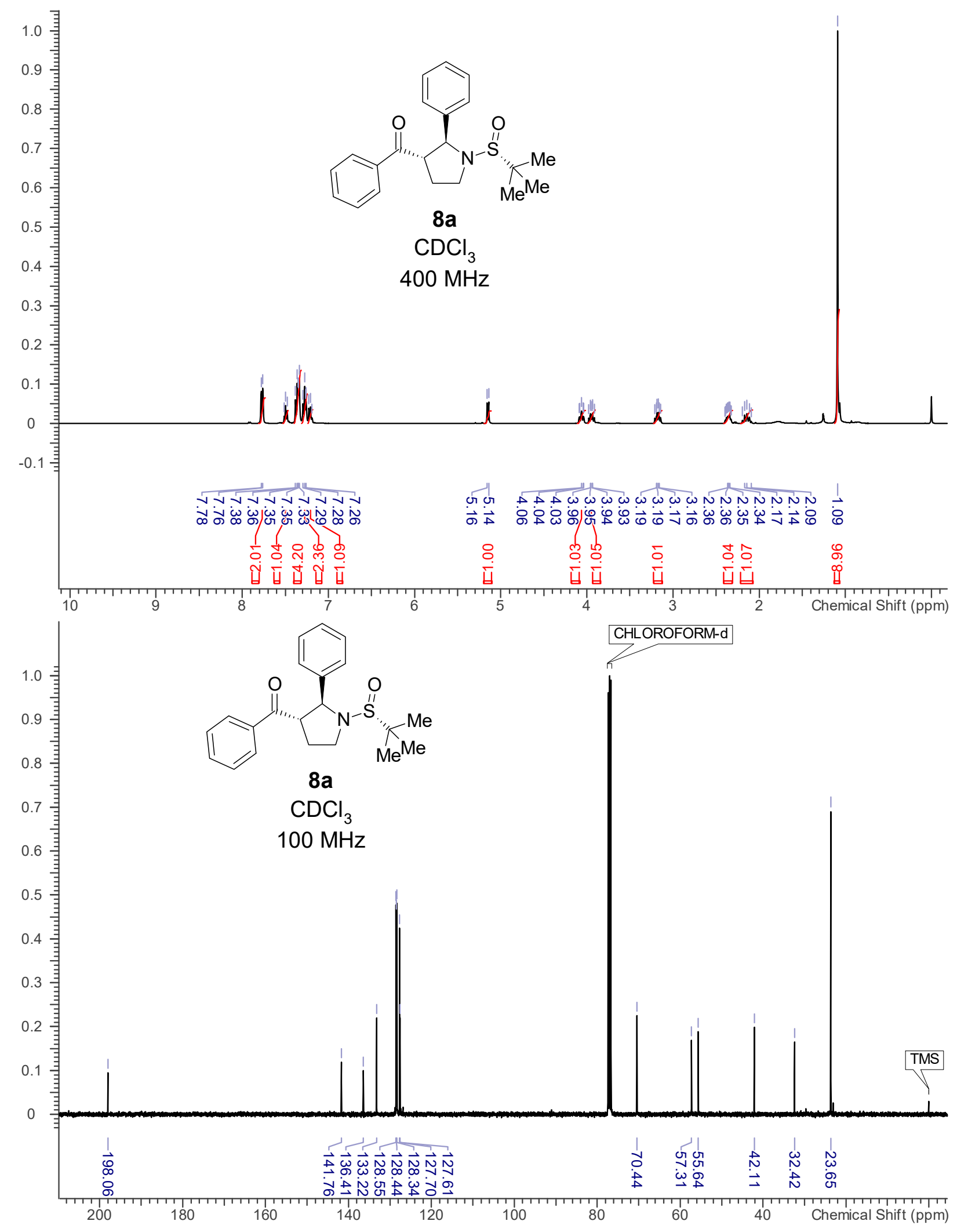




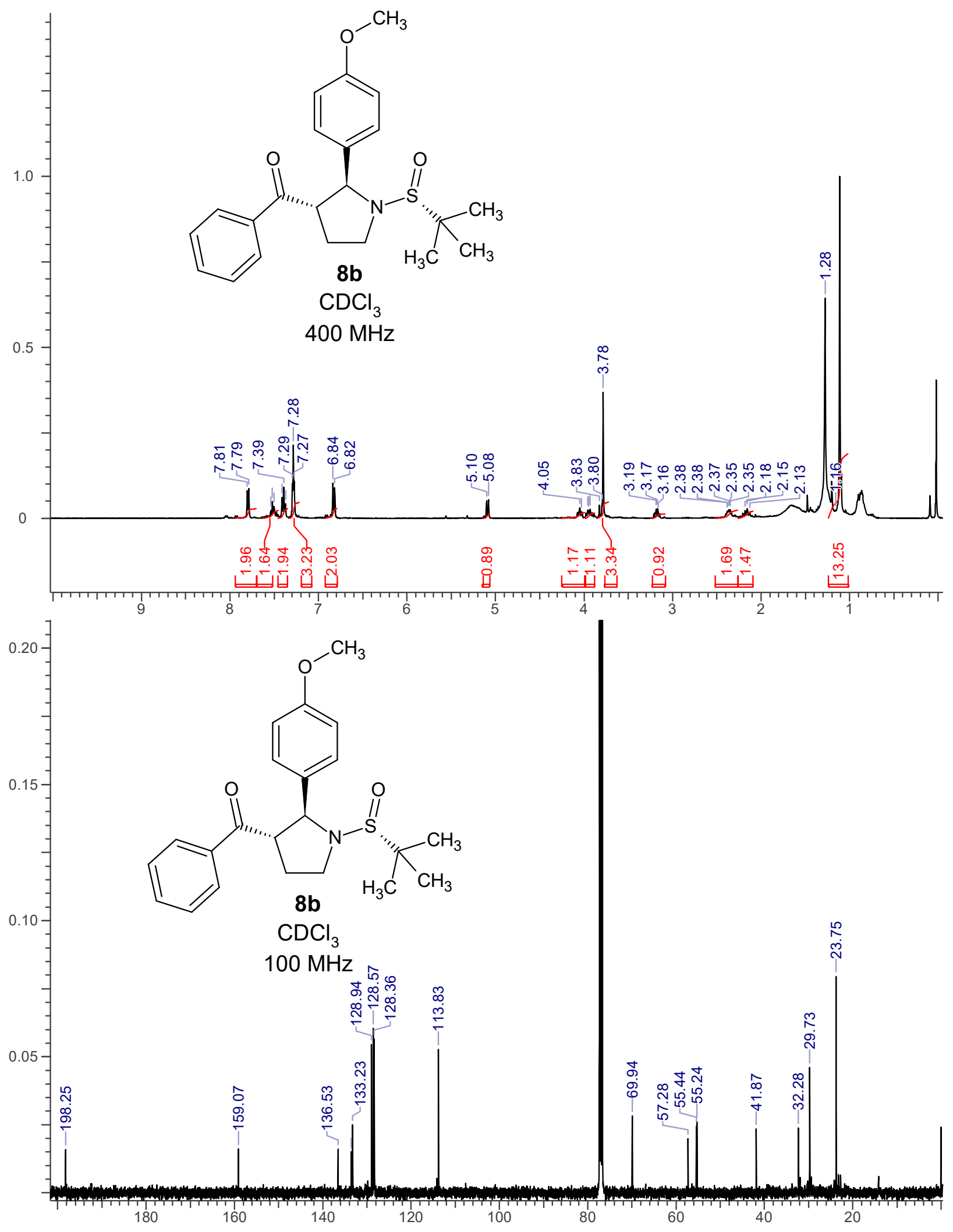



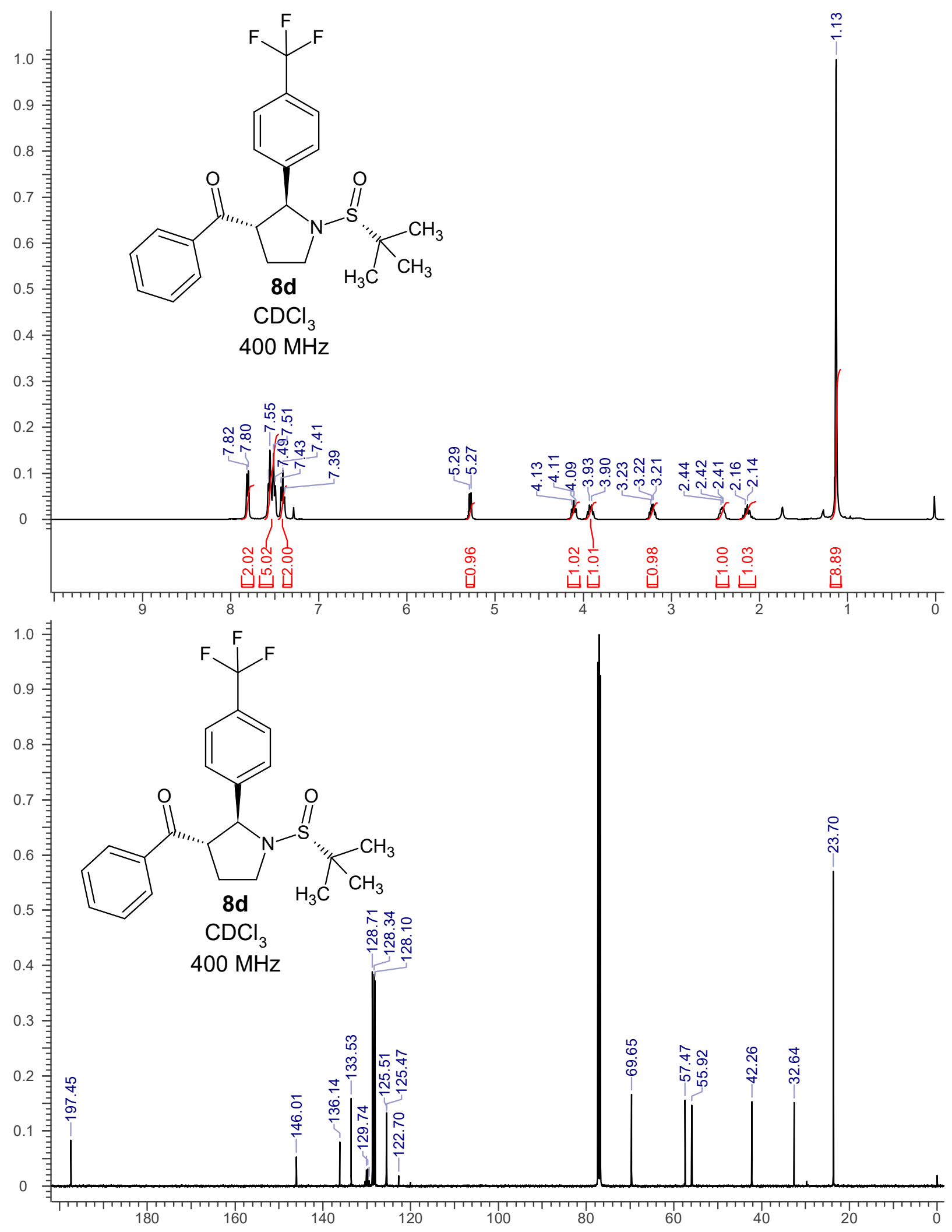


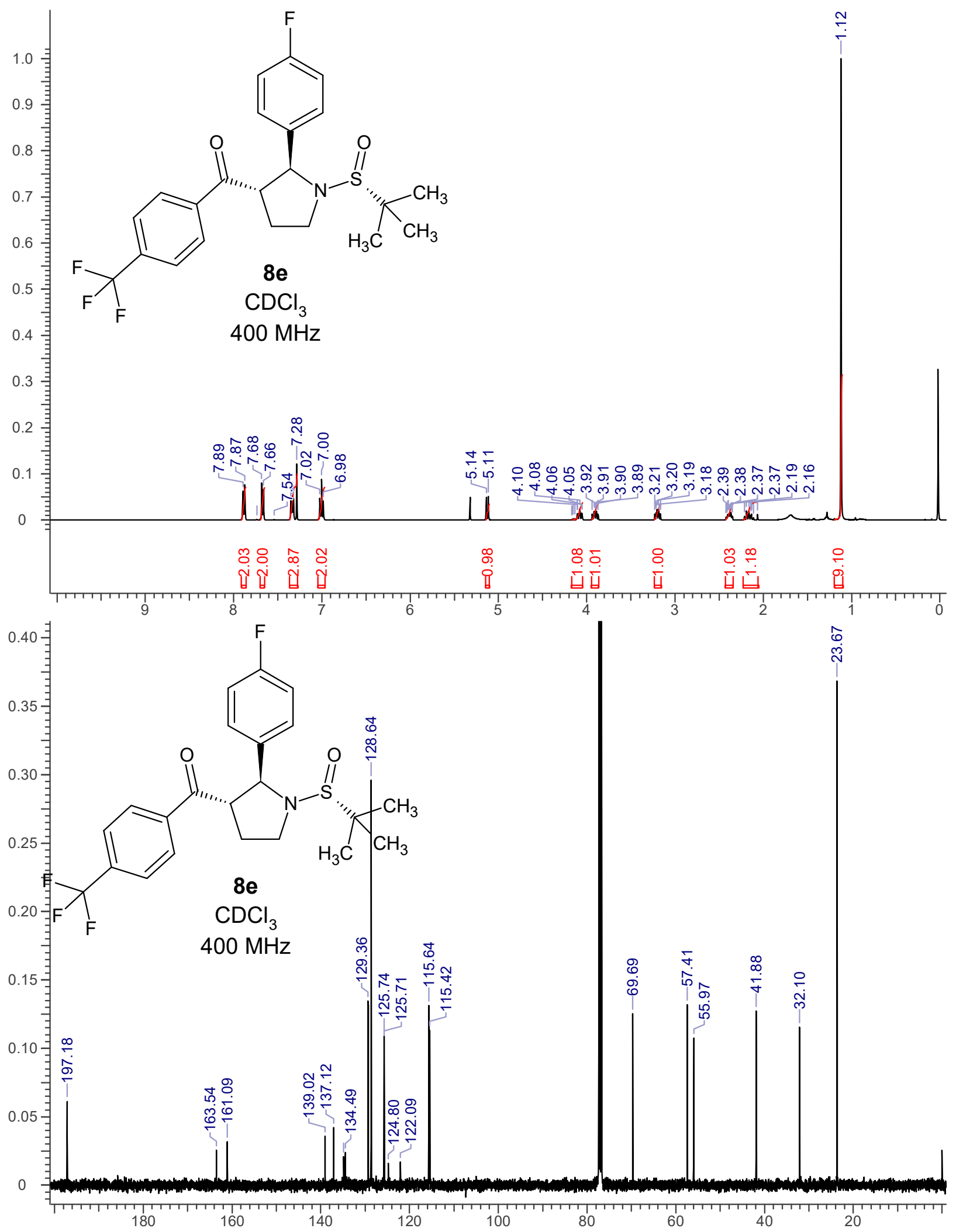




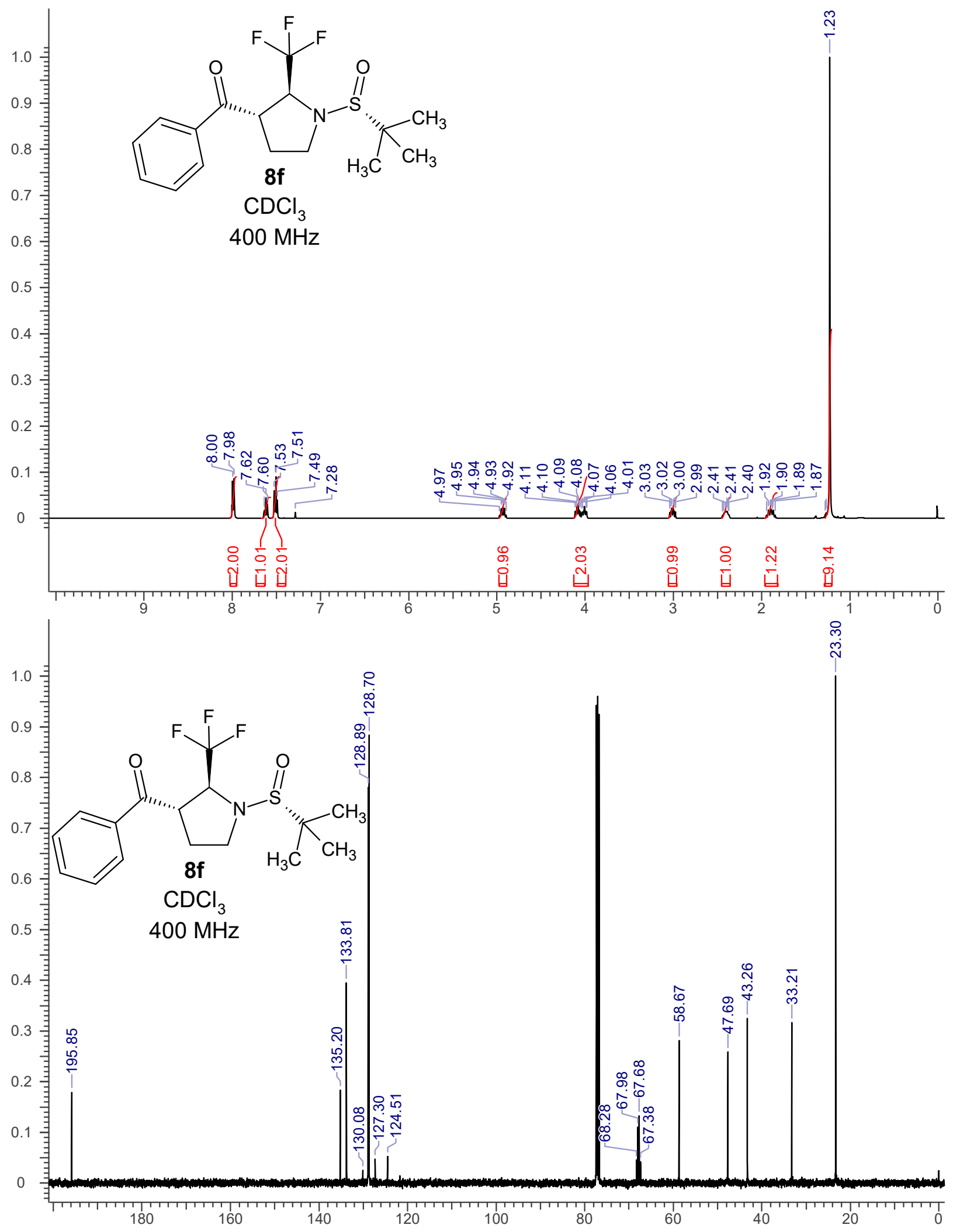




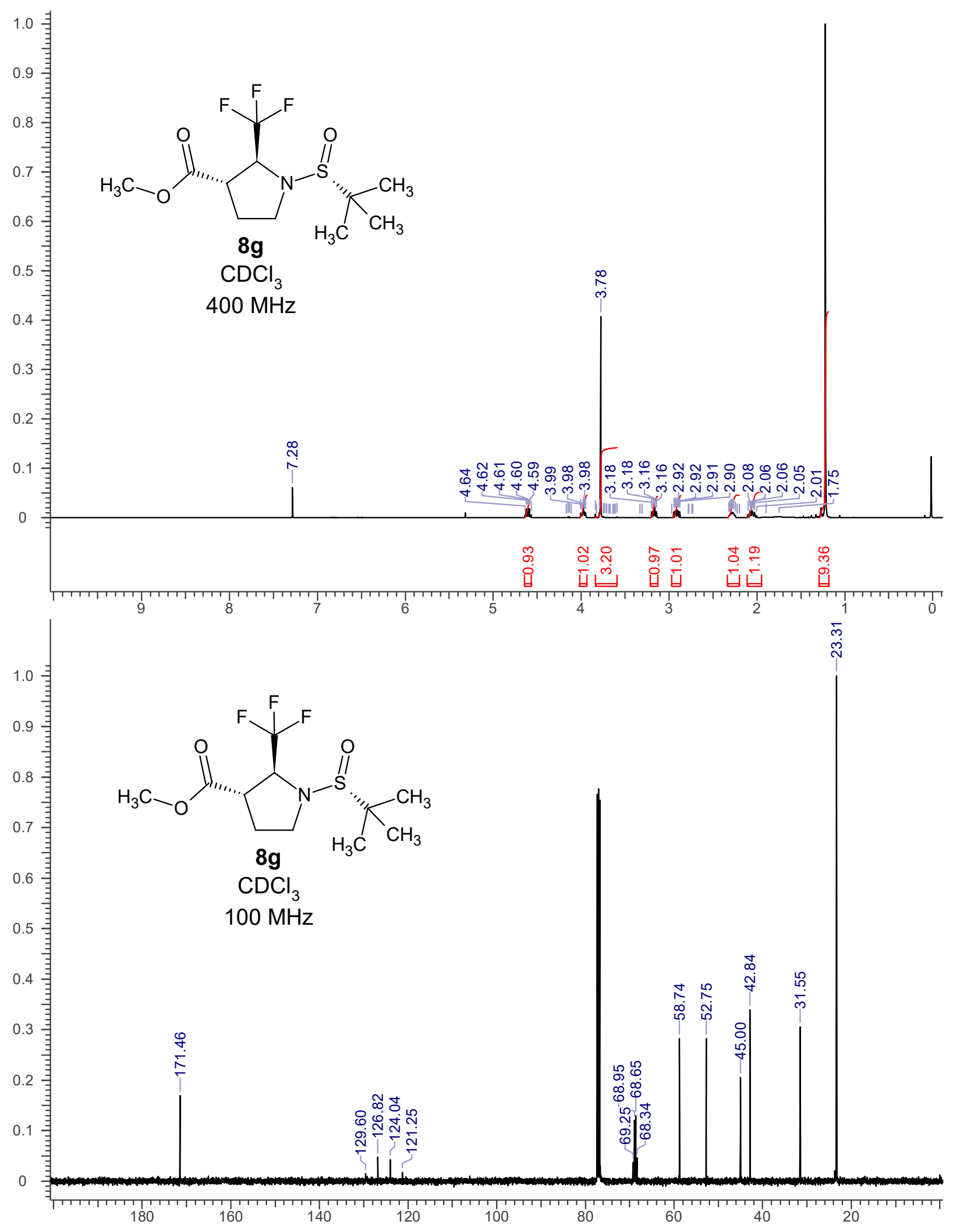




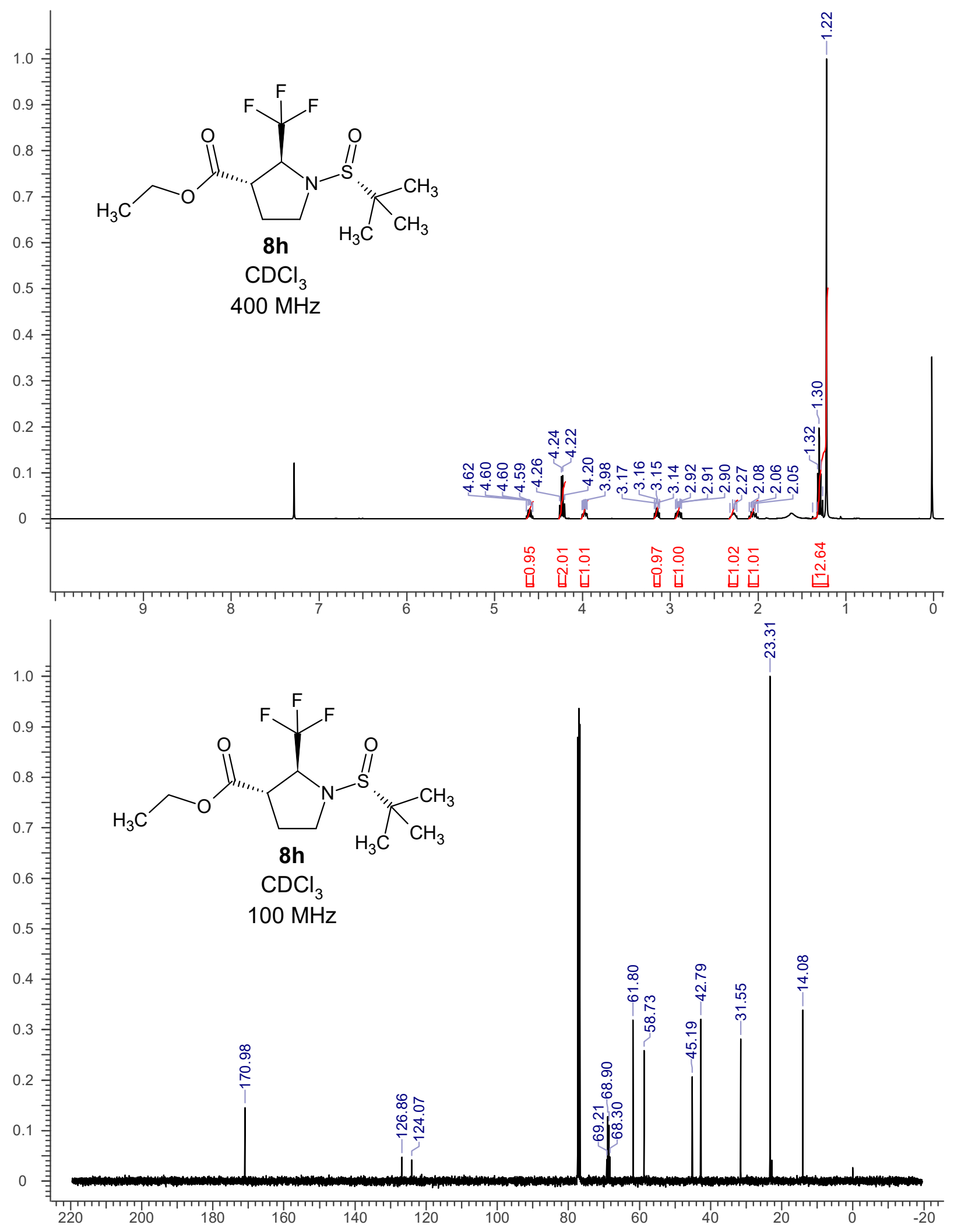




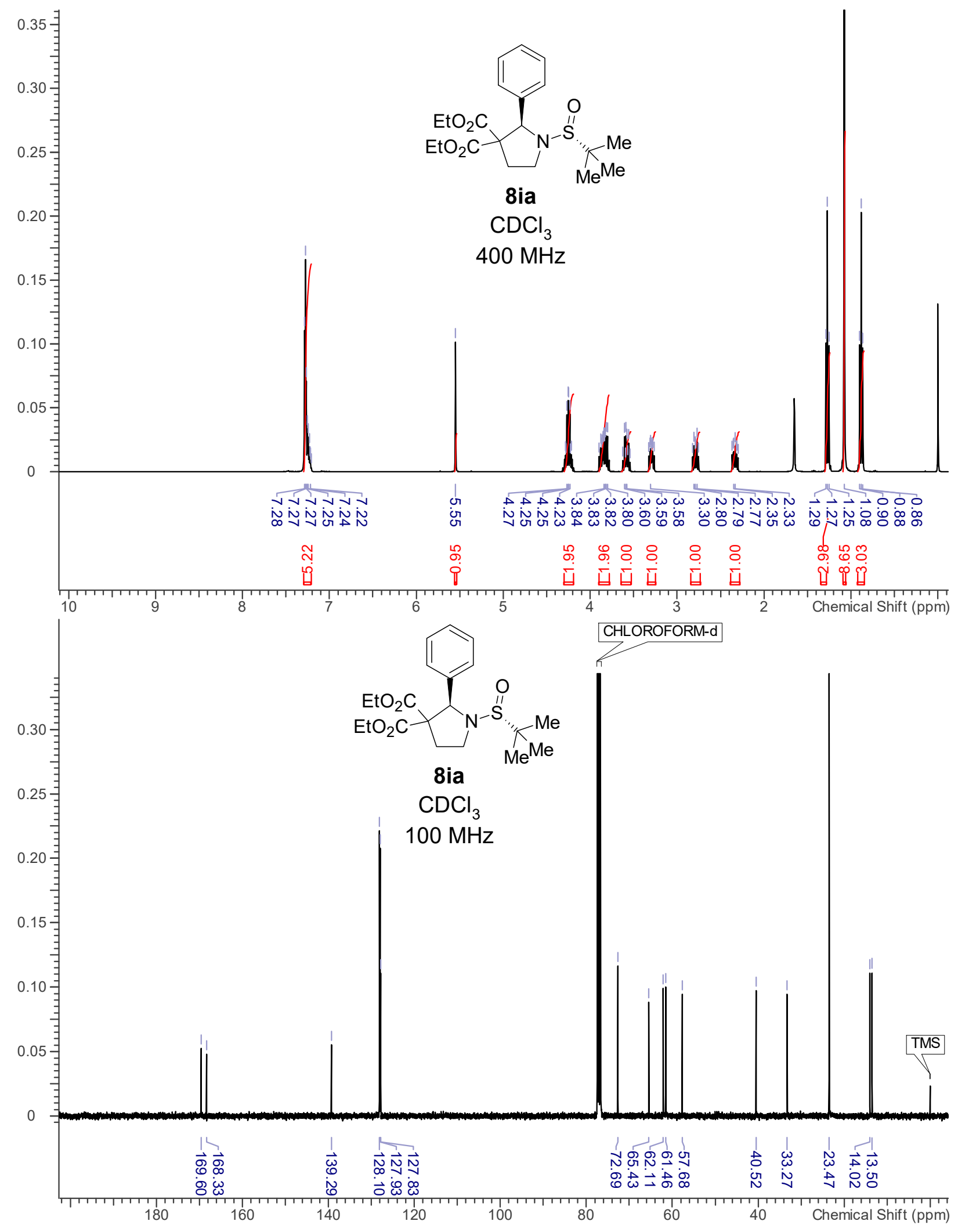




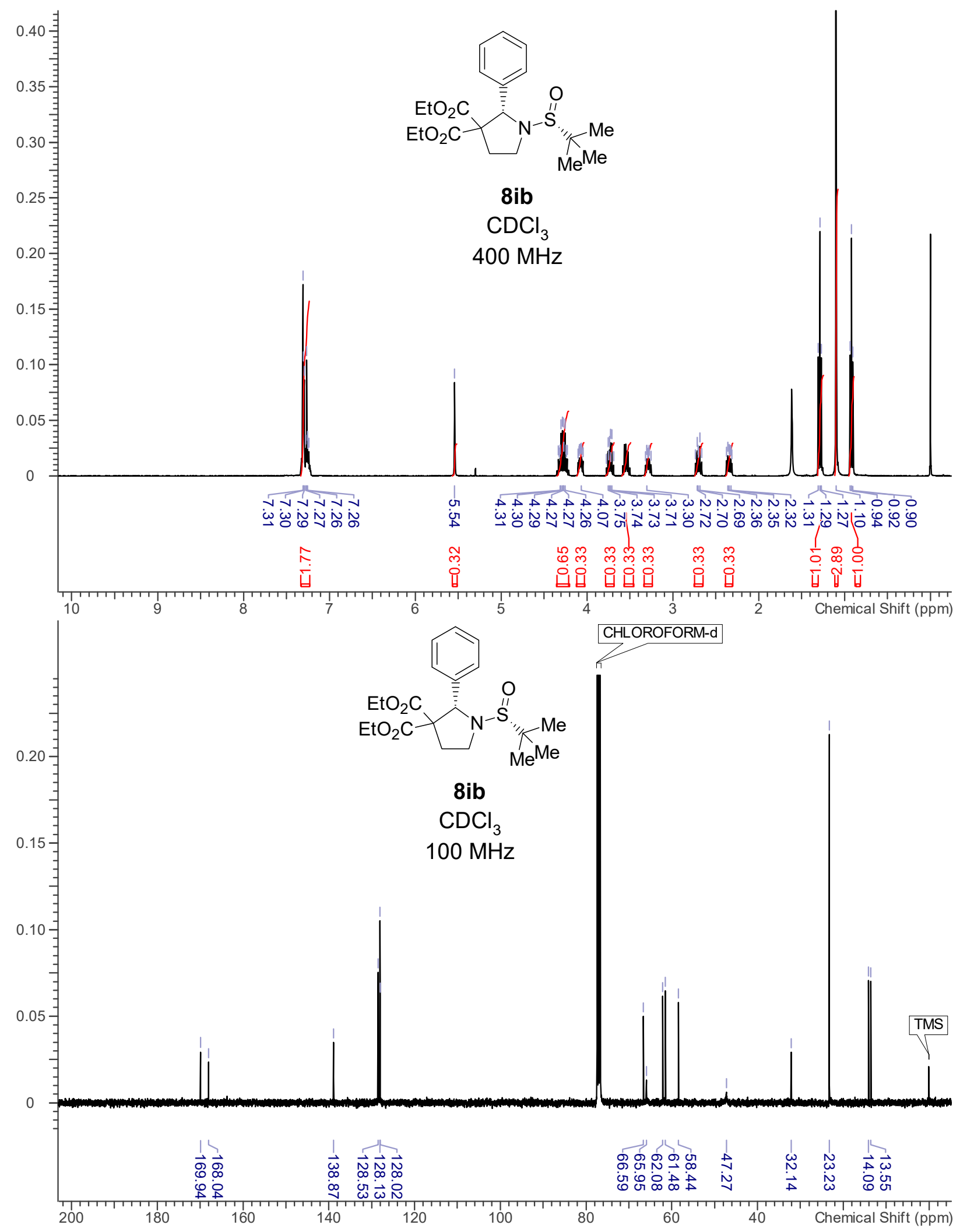




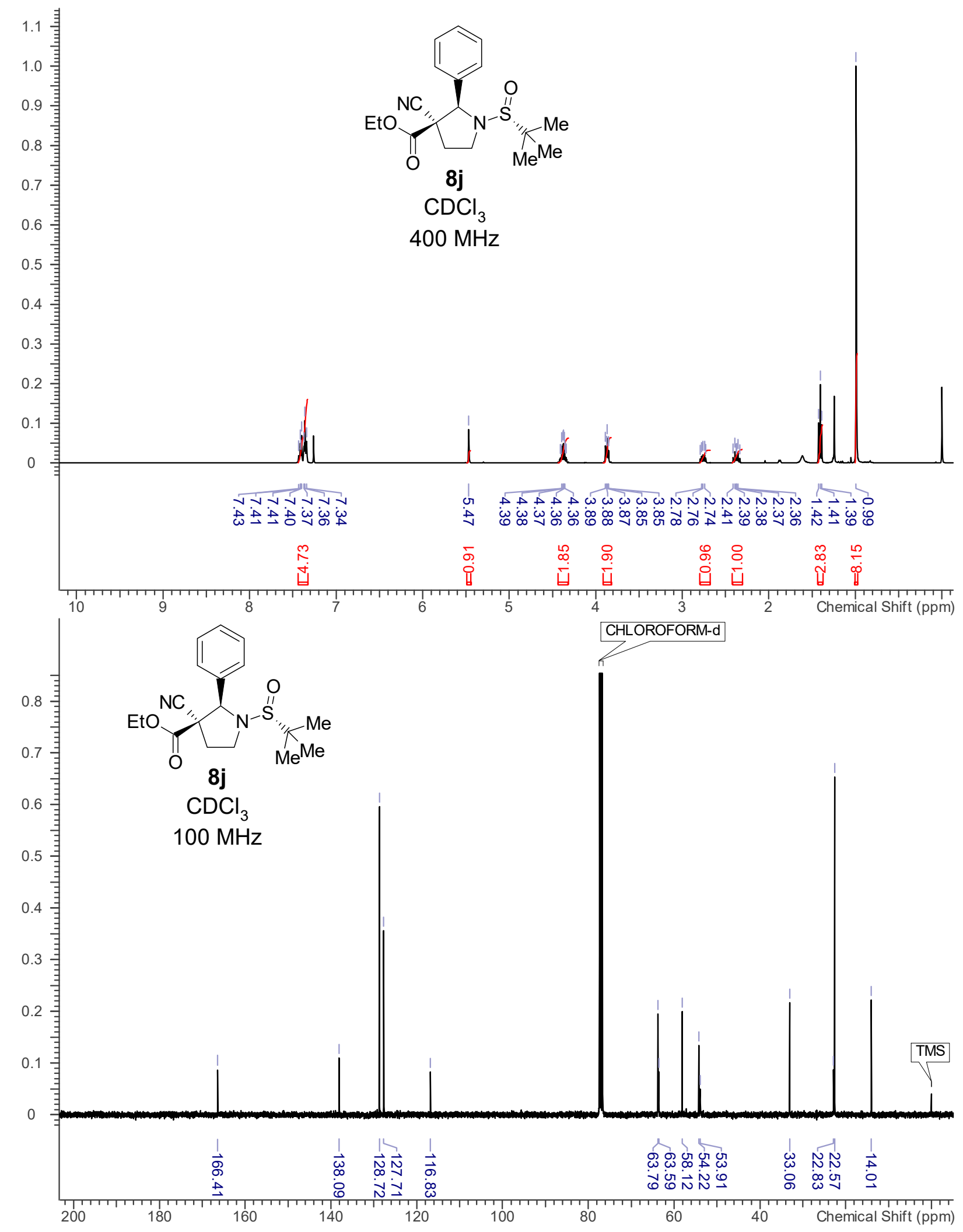




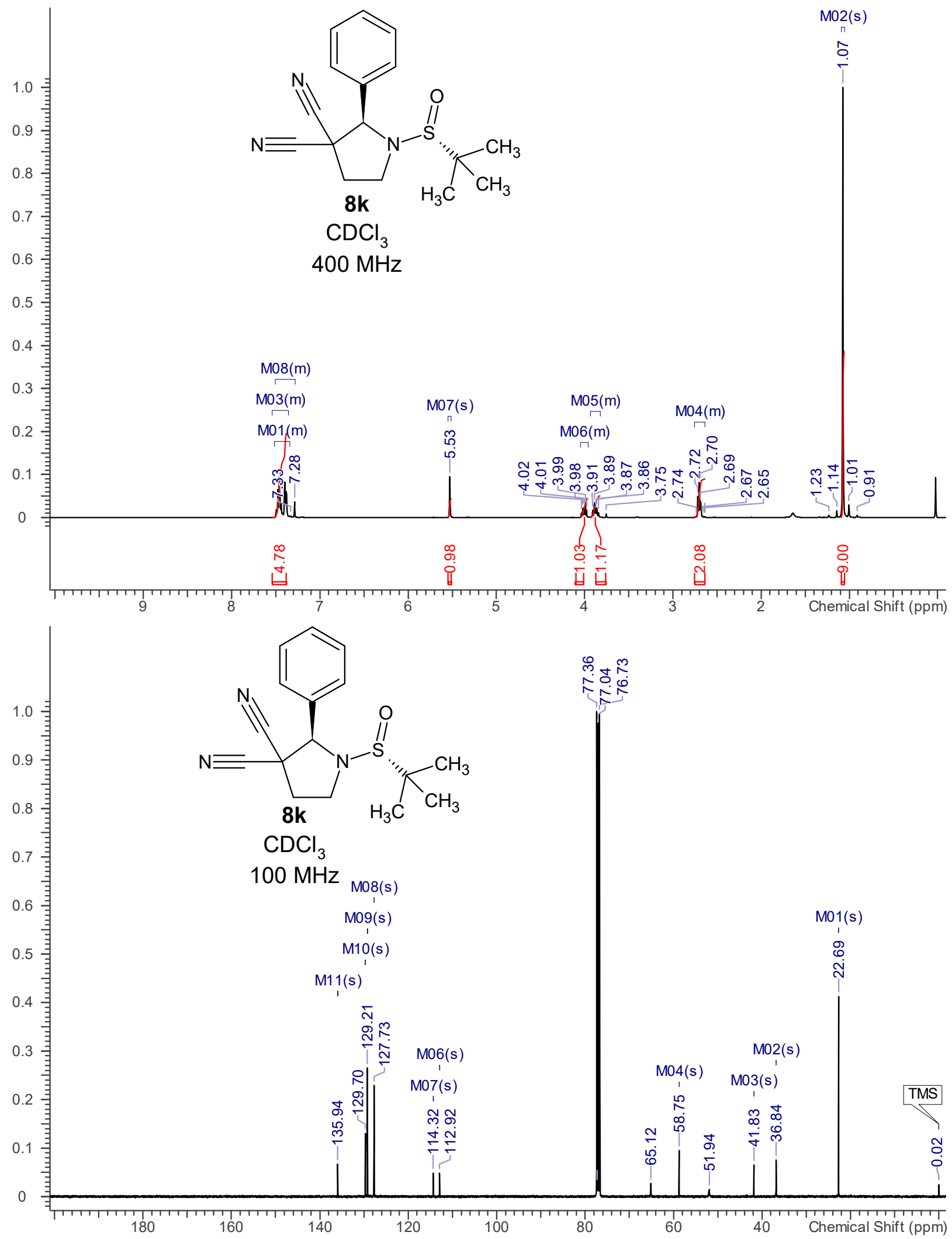




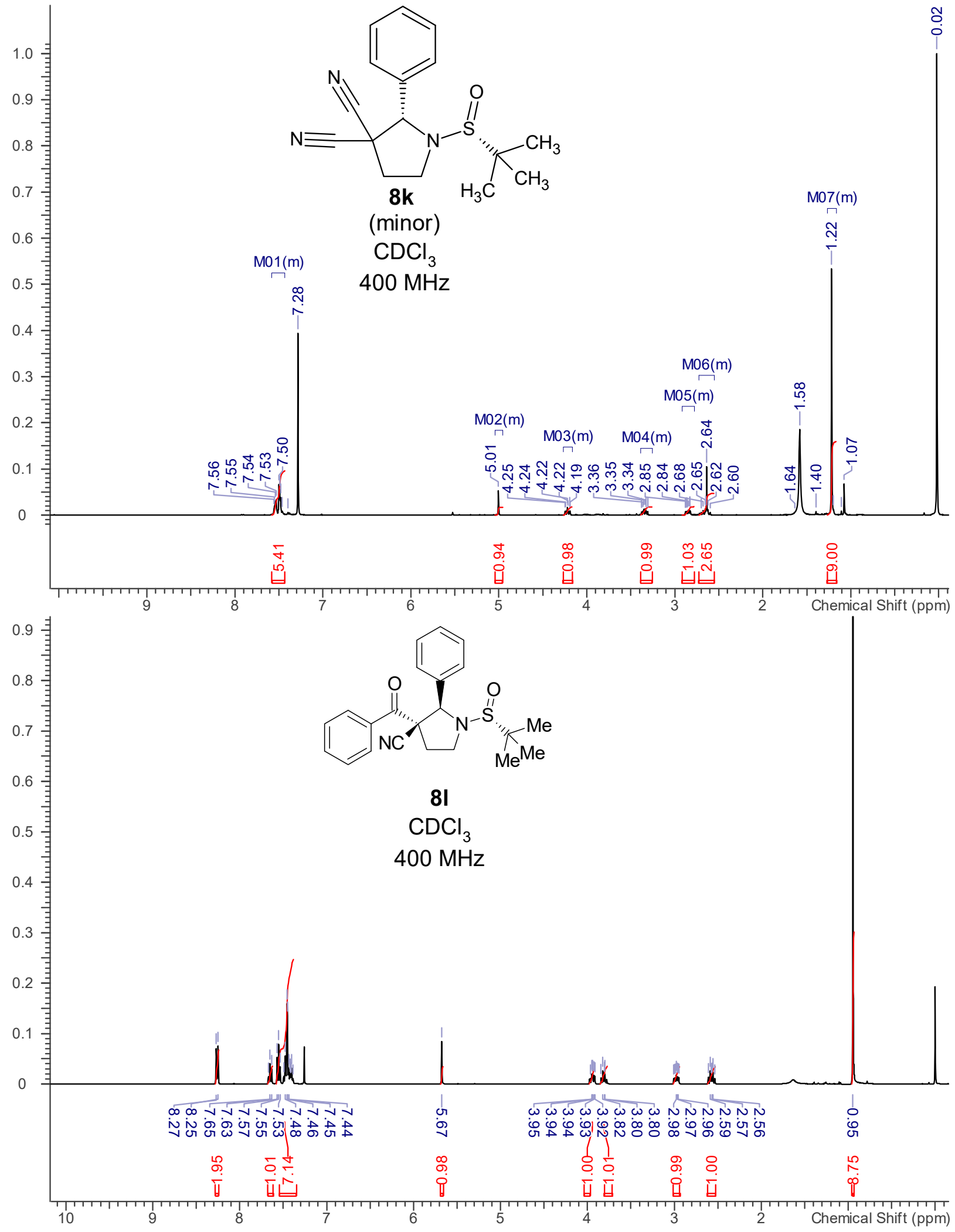




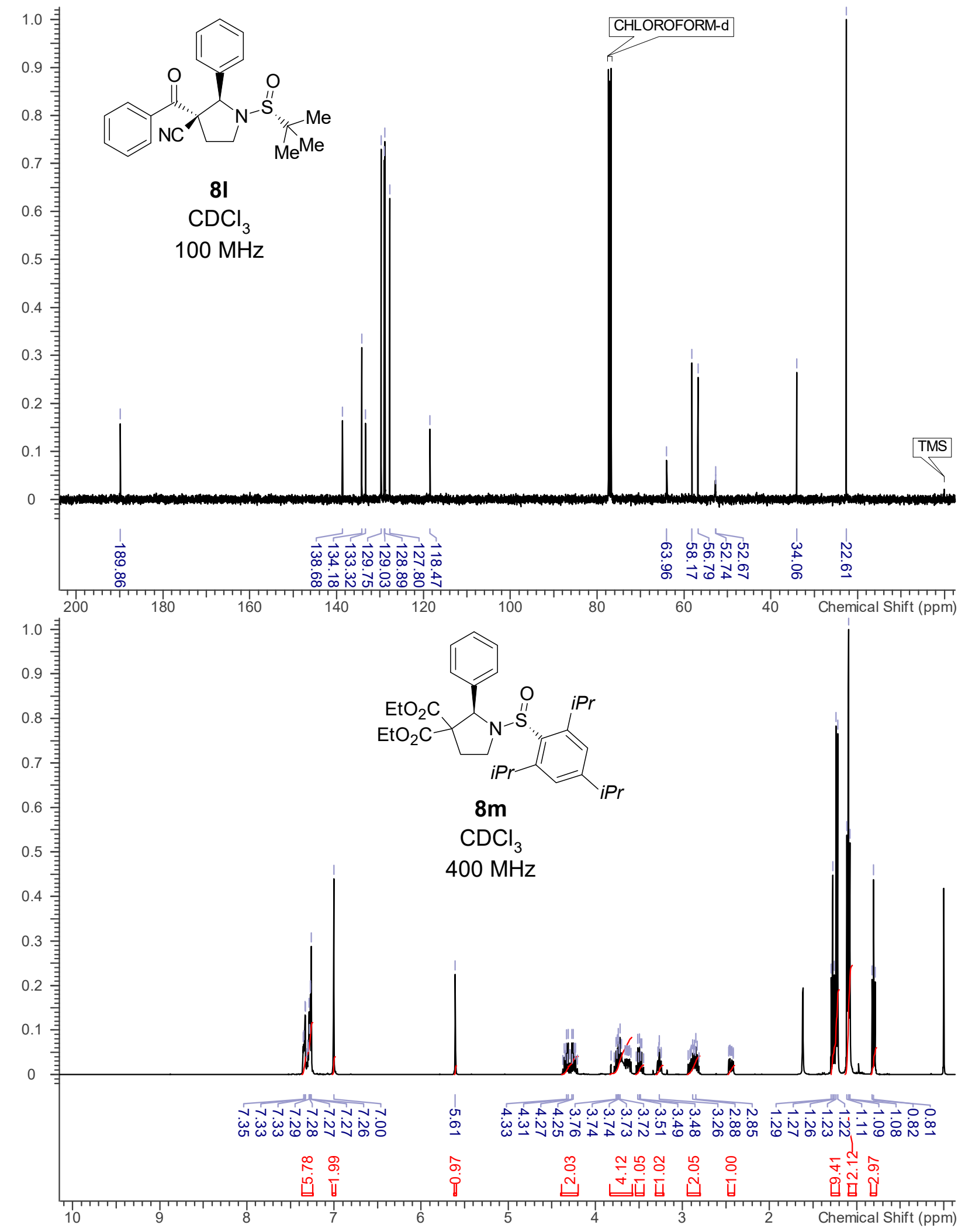



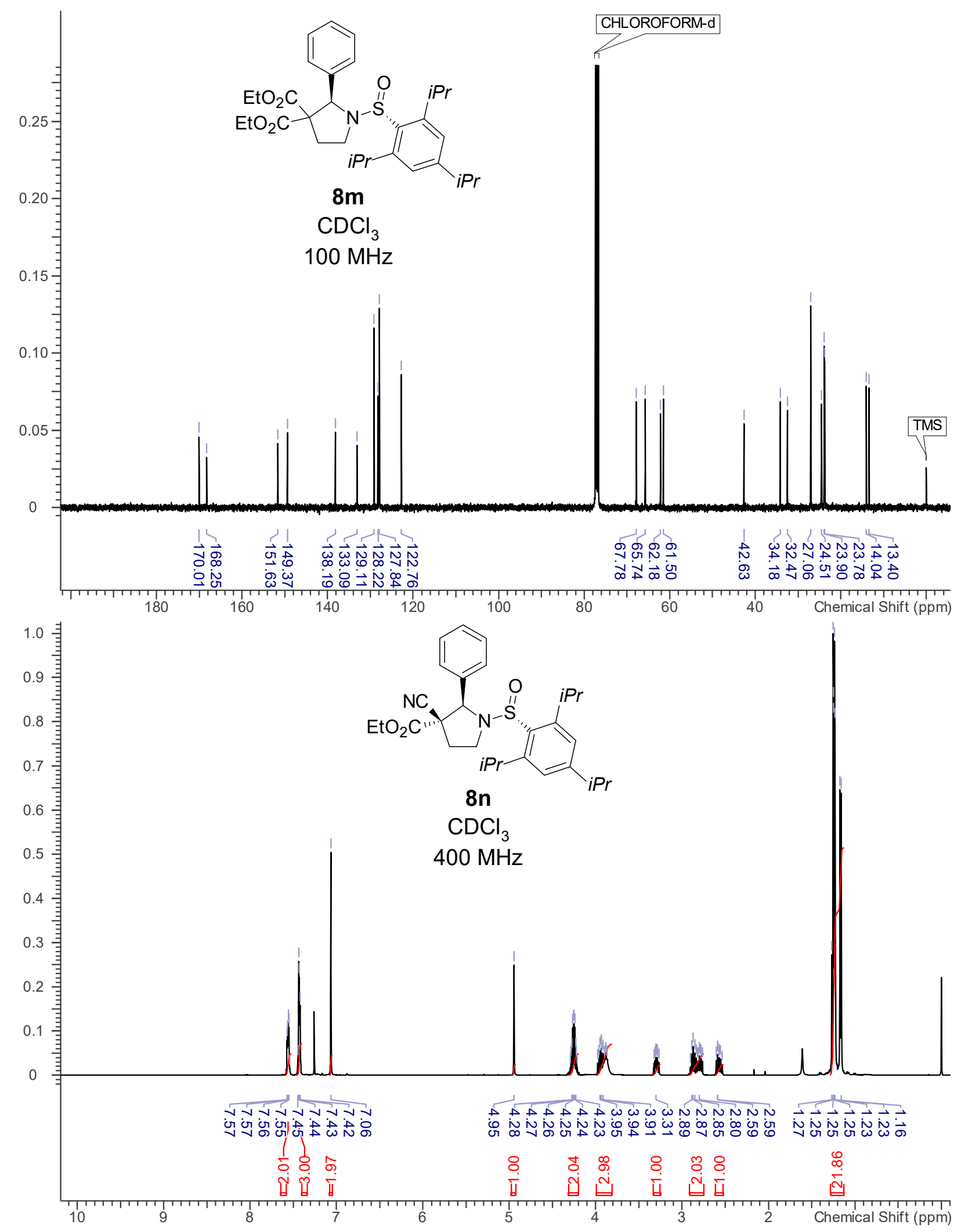


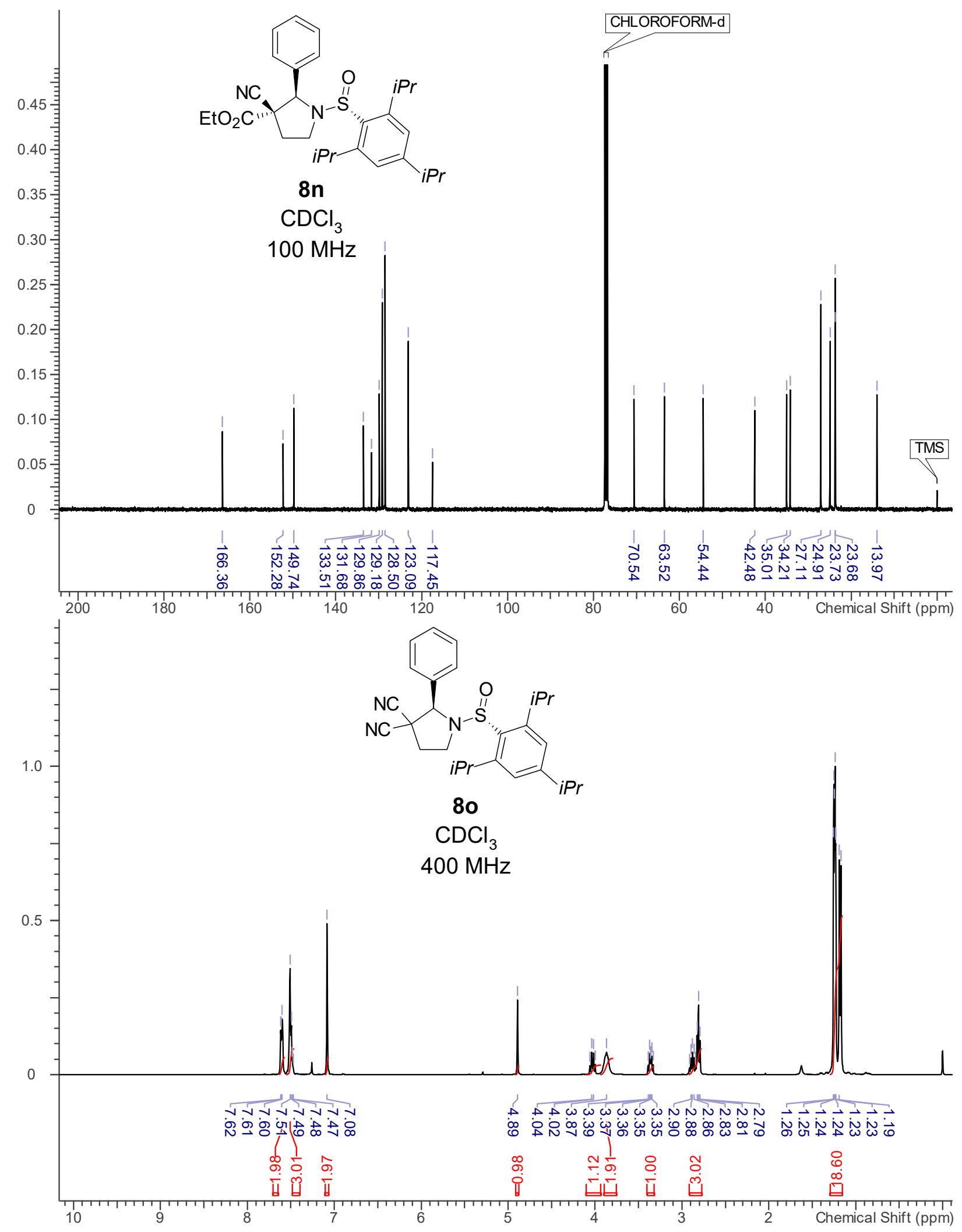








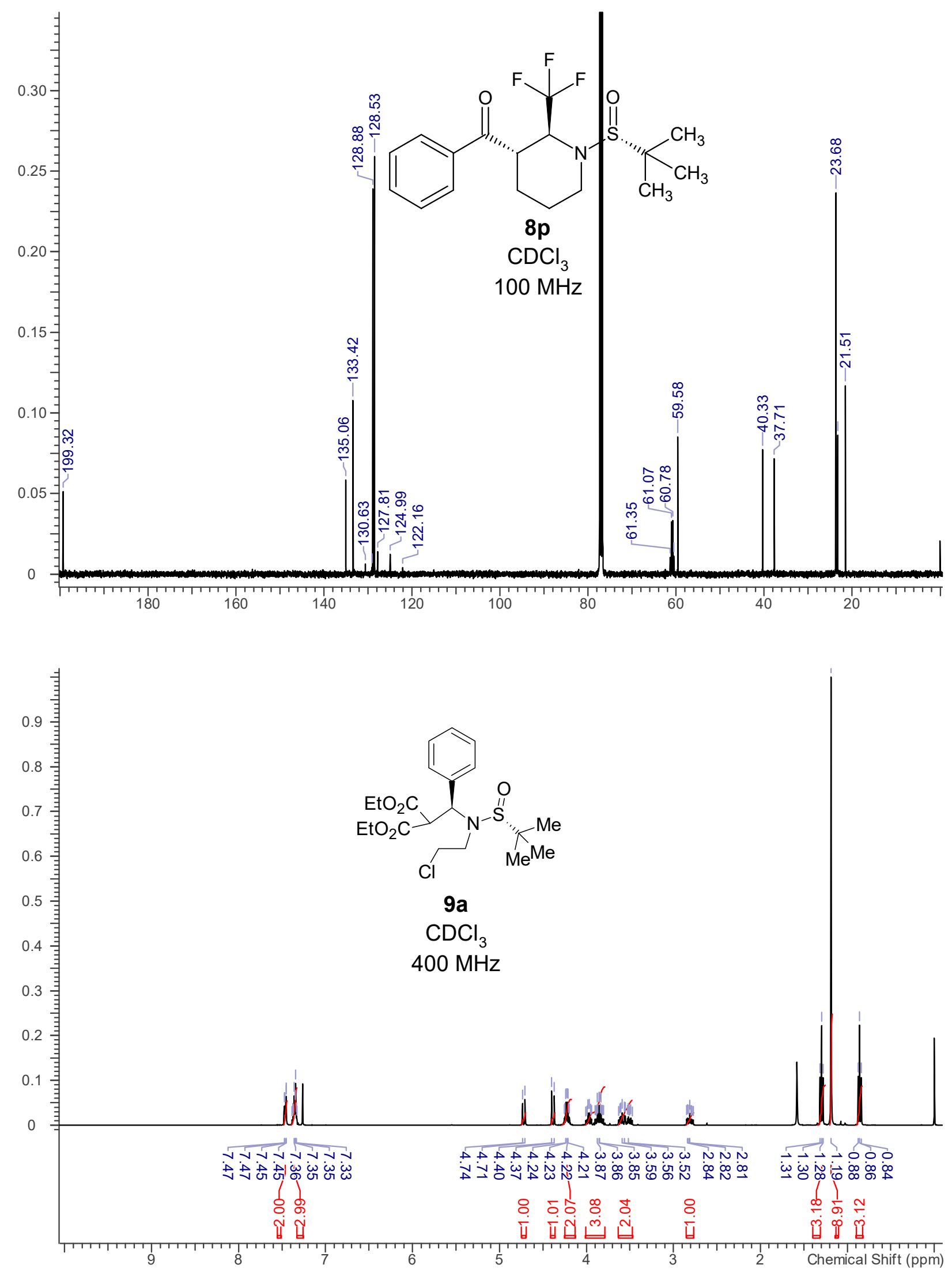


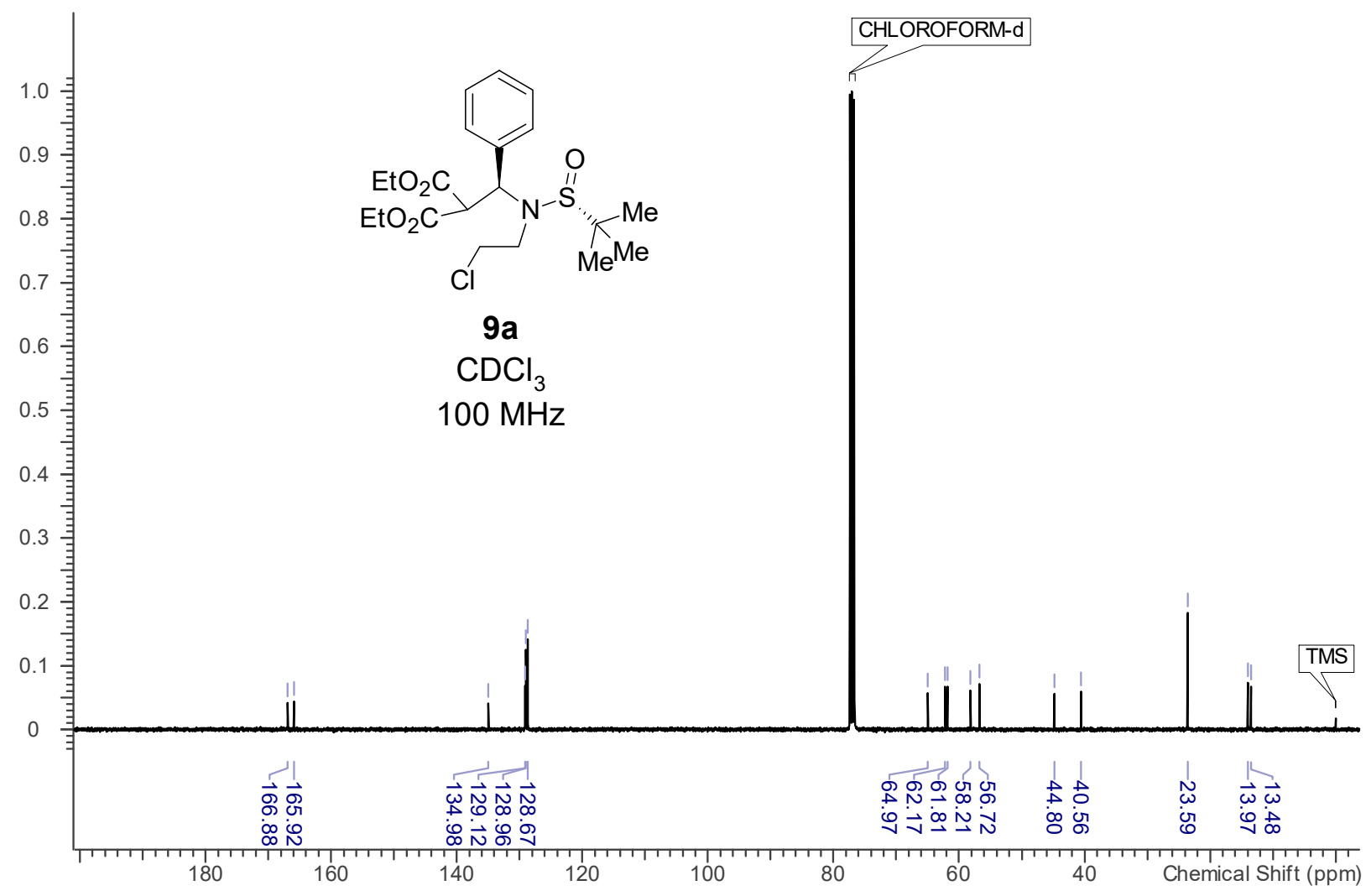

